\title{
Five NeW SPECIES OF PRISTIMANTIS (ANURA: StRABOMANTIDAE) from the CoAstal Cloud forest of the Península de PARia, VENEZUELA
}

\author{
Hinrich Kaiser ${ }^{1}$, César L. Barrio-Amorós ${ }^{2}$, Gilson A. Rivas ${ }^{3}$, Claus Steinlein ${ }^{4} \&$ \\ Michael Schmid ${ }^{5}$
}

\footnotetext{
${ }^{1}$ Department of Biology, Victor Valley College, 18422 Bear Valley Road, Victorville, California 92395, USA

${ }^{1}$ Department of Vertebrate Zoology, National Museum of Natural History, Smithsonian Institution, Washington, D.C. 20013, USA

${ }^{2}$ Doc Frog Expeditions, Apartado Postal 220-8000, San José, Perez Zeledon, San Isidro del General 11901, Costa Rica

${ }^{3}$ Museo de Biologia, Facultad Experimental de Ciencias, La Universidad del Zulia, Apartado Postal 526, Maracaibo 4011, Maracaibo, Venezuela

4,5 Institut für Humangenetik, Universität Würzburg, Biozentrum Am Hubland, 97074 Würzburg, Germany

${ }^{1}$ hinrich.kaiser@vvc.edu (corresponding author), ${ }^{2}$ cesarlba@yahoo.com, ${ }^{3}$ grivas@fec.luz.edu.ve,

${ }^{4}$ claus.steinlein@biozentrum.uni-wuerzburg.de, ${ }^{5}$ m.schmid@biozentrum.uni-wuerzburg.de
}

\begin{abstract}
Fieldwork in the cloud forest of Venezuela's remote Península de Paria in 2001 resulted in the collection of several specimens that could unquestionably be classified as members of the genus Pristimantis. Subsequent analysis of comparative material in museum collections brought the total number of specimens to 44 , and these collectively represent five new species. Two of these species, P. geminus sp. nov. and P. nubisilva sp. nov., have phenotypes remarkably similar to the Trinidadian $P$. urichi, supporting a prediction that Pristimantis from easternmost Venezuela may have given rise to Trinidadian forms. Pristimantis hoogmoedi sp. nov. is easily identified by its large size and red eyes. Two of the species, P. longicorpus sp. nov. and P. pariagnomus sp. nov., are very distinct morphologically but are known only from the holotypes. The former is characterized by an elongate body form supported by relatively short limbs, whereas the latter has very distinctive hand morphology and is likely the smallest Venezuelan frog. Chromosome banding studies of $P$. nubisilva sp. nov. and $P$. hoogmoedi sp. nov. revealed chromosome numbers of $2 n=36$ and $2 n=26$, respectively, with an unusual submetacentric fusion chromosome $11 ; 18$ in some males of the former and a unique meiotic pairing of chromosomes in males of the latter. All five species can be readily distinguished by their osteology, such as by the extent of the sphenethmoid and features on the roof of the mouth, as well as by the shape and rearrangement of mesopodial elements. The unexpectedly high diversity of Pristimantis in this region, along with high endemism of amphibians and reptiles in general, underscores the position of the Península de Paria as a center for frog biodiversity in Venezuela. The similarity of these Paria species to Pristimantis from Trinidad, Tobago and the central Cordillera de la Costa represents a tangible piece of evidence for the close biogeographic link of the anuran fauna of these landmasses.
\end{abstract}

Keywords: Biogeography, chromosomes, new species, osteology, Península de Paria, Pristimantis, taxonomy, Trinidad, Tobago, Venezuela.

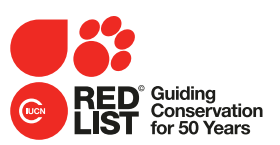

DOI: http://dx.doi.org/10.11609/JoTT.04197.7047-88 | ZooBank: urn:Isid:zoobank.org:pub:D176BOE5-F6F3-4ADB-8842-C9DAB45EEB78

Editor: Anonymity requested.

Date of publication: 17 April 2015 (online \& print)

Manuscript details: Ms \# 04197 | Received 11 December 2014 | Final received 23 February 2015 | Finally accepted 31 March 2015

Citation: Kaiser, H., C.L. Barrio-Amorós, G.A. Rivas, C. Steinlein \& M. Schmid (2015). Five new species of Pristimantis (Anura: Strabomantidae) from the coastal cloud forest of the Península de Paria, Venezuela. Journal of Threatened Taxa 7(4): 7047-7088; http://dx.doi.org/10.11609/JoTT.04197.7047-88

Copyright: @ Kaiser et al. 2015. Creative Commons Attribution 4.0 International License. JoTT allows unrestricted use of this article in any medium, reproduction and distribution by providing adequate credit to the authors and the source of publication.

Funding: This research received partial funding through grants from the Volkswagen Foundation, Hannover, Germany (Grant I/72 515) to Michael Schmid, and from the College of Arts and Sciences and the University Research Council at La Sierra University, Riverside, California, USA, to Hinrich Kaiser.

Competing Interest: The authors declare that no competing interests influenced the preparation and presentation of this manuscript.

For Acknowledgements, Author Details, Author Contribution and Spanish Abstract see end of this article.

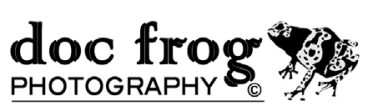

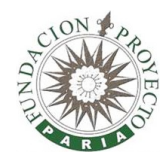

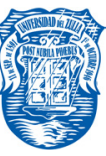

Smithsonia National Museum of Natural Histor
ISSN 0974-7907 (Online) ISSN 0974-7893 (Print) \\ OPEN ACCESS}




\section{INTRODUCTION}

The Península de Paria in extreme northeastern Venezuela (Image 1 ) is an extension of a chain of high coastal mountains (elevations reaching nearly $2800 \mathrm{~m}$ ) that span much of northern Venezuela and extend into the adjacent islands of Trinidad and Tobago (Frost $\&$ Snoke 1989). This region has been variously called the Cordillera de la Costa (e.g., Barrio-Amorós \& Kaiser 2008), the Coastal Range (e.g., Rivero 1961) or the Región Orocostense (Barrio-Amorós 1998), and its remote far eastern continental reaches have hitherto received only sporadic attention from herpetologists. Despite relatively limited research, the Península de
Paria's steeply sloping hillsides with their verdant foliage and their lush cloud forest at higher elevations (with an upper altitudinal limit of $1300 \mathrm{~m}$; Image 2), have been noted for a diverse endemic herpetofauna. DonosoBarros $(1965 a, b)$ reported results from Cerro Azul $\left(10.6939^{\circ} \mathrm{N} \& 61.9467^{\circ} \mathrm{W}\right.$; Image 1 inset) and described Mannophryne riveroi (Donoso-Barros, 1965a), the first endemic frog from the area, followed by the nominate subspecies of Gonatodes ceciliae Donoso-Barros, 1966, the first endemic lizard. Stephen Edwards explored Cerro Azul in the research leading to his unpublished dissertation on dendrobatid frogs (see Edwards 1974) and recognized a distinct species that was described many years later as Mannophryne venezuelensis

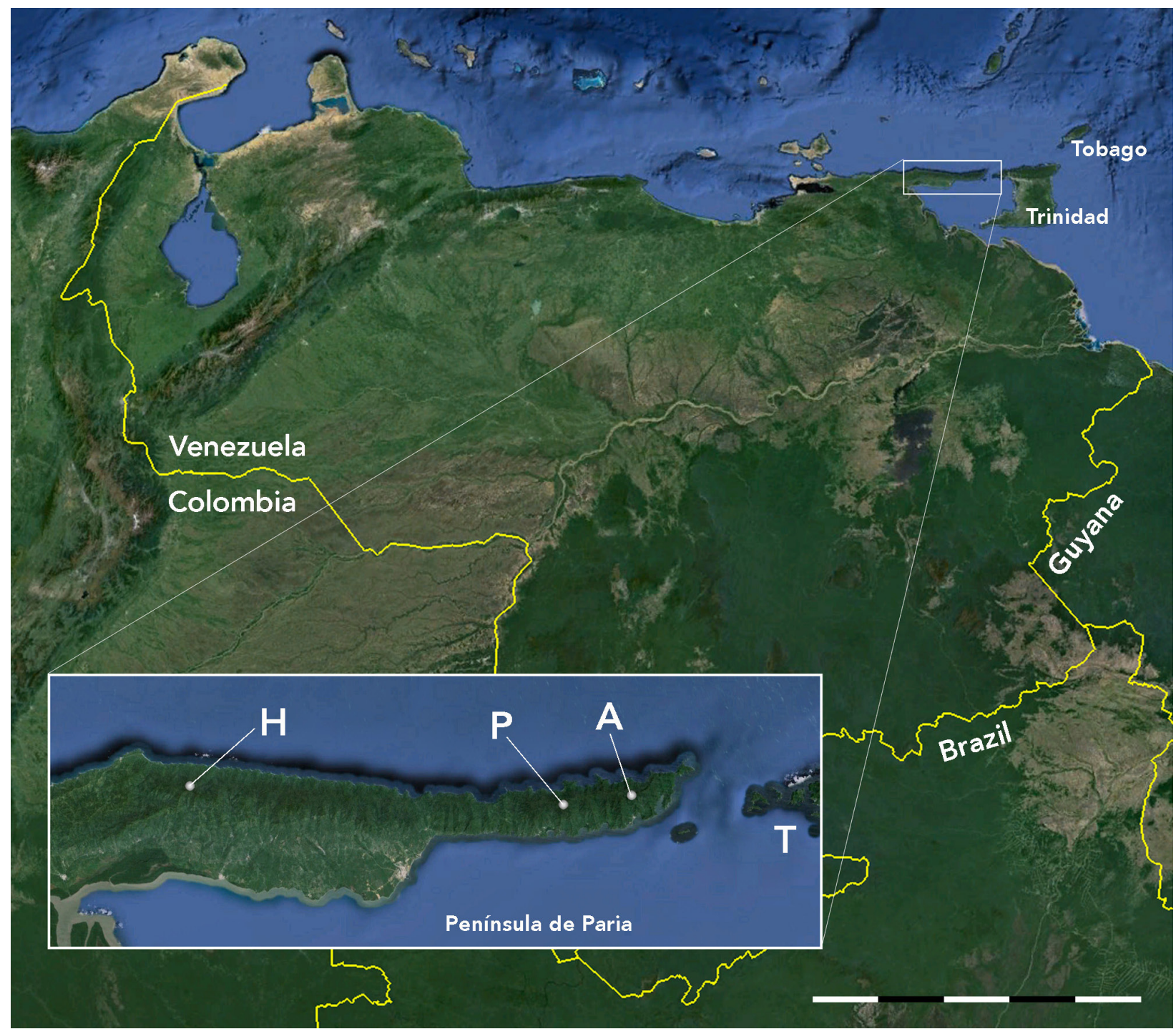

Image 1. Satellite map of Venezuela and surrounding areas. The location of the Península de Paria is indicated by the rectangle in the upper right-hand corner of the map. The inset shows a close-up of the peninsula and adjacent Trinidad ( $T$ ), with three important centers of endemism indicated by labeled points: Cerro Humo (H), Cerro Patao (P), and Cerro Azul (A). Scale $=500 \mathrm{~km}$. Map produced from Google Earth images. 


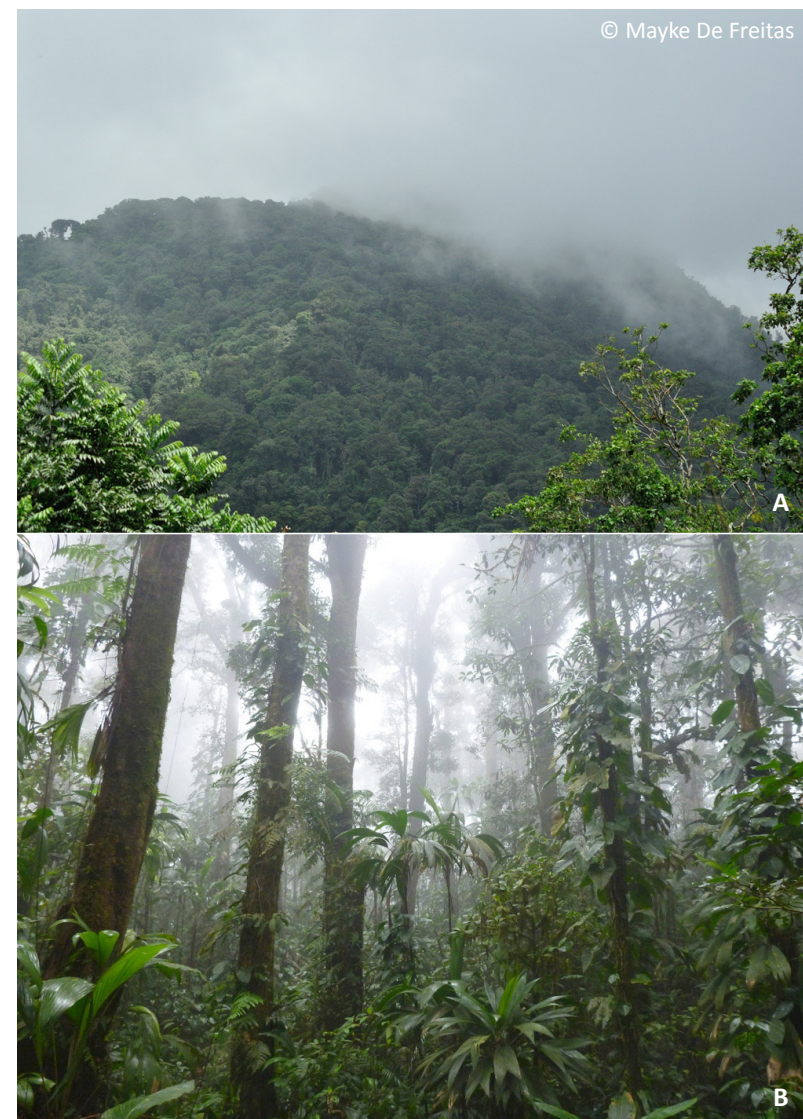

Image 2. Photographs of Cerro Humo in the Península de Paria, provided as an example for the cloud forest habitat in which the five new species of Pristimantis were discovered. (A) A rare view of the nearly unobstructed peak of Cerro Humo taken from the village of Las Melenas. (B) Dense cloud forest habitat on the southern versant of Cerro Humo, near the type locality for the five new species of Pristimantis.

by Manzanilla et al. (2007). Two centrolenid frogs, Celsiella vozmedianoi (Ayarzagüena \& Señaris, 1997) and Vitreorana castroviejoi (Ayarzagüena \& Señaris, 1997), were discovered on Cerro Humo $\left(10.7073^{\circ} \mathrm{N} \&\right.$ $62.6284^{\circ} \mathrm{W}$; Image 1 inset, Image 2). Rivas et al. (1999) described the microteiid Anadia pariaensis, MijaresUrrutia et al. (2000) described the gymnophthalmid lizard Euspondylus monsfumus, and Barrio-Amorós et al. (2006) named Allobates caribe, all from the same area. Other herpetologists visited the Península de Paria and made important collections (e.g., Stefan Gorzula in 1978, Jose Ayarzagüena in 1996) but no additional species have been described from their material. With the completion of more extensive collections by several Venezuelan and international research teams, additional species and observations on poorly known species were recently published (e.g., Anadia pariaensis-Rivas et al. 2012; Strabomantis biporcatus-Barrio-Amorós \&
Kaiser 2008; Mannophryne riveroi-Barrio-Amorós et al. 2010a; Riama rhodogaster-Rivas et al. 2005; Taeniophallus nebularis-Schargel et al. 2005). This study continues the trend of discovery for the Paria area with the description of five hitherto unrecognized species of Pristimantis.

\section{MATERIALS AND METHODS}

\section{Collection}

The specimens we used for this study come from several expeditions to the Península de Paria, including some collected by Stefan Gorzula and Marinus Hoogmoed in the late 1970s. As a consequence, there are differences in the data we were able to obtain. For some specimens, we have only field notes to document their coloration in life. Only for two of the new species was it possible to obtain chromosome spreads, even though we collected suitable samples for all four of the species we personally encountered.

Those specimens we personally collected (as listed in the respective species accounts) were obtained by hand capture on 03-09 September 2001 during day and night surveys on the slopes of Cerro Humo $\left(10.7073^{\circ} \mathrm{N}\right.$ \& $62.6284^{\circ} \mathrm{W}$; Image 1 inset). Due to time constraints, our survey protocol conformed to a standard visual encounter survey (Heyer et al. 1994), conducted from forest paths and along the courses of small creeks during both day- and nighttime hours. All searched potential refugia were restored as closely as possible to their original state. When possible, we let vocalizations guide our search by night. For all captures, we recorded basic collecting information (date, time, altitude, circumstances of capture, GPS coordinates). GPS coordinates were verified using Google Earth software.

\section{Processing}

Specimens were placed individually into sizeappropriate screw-top plastic containers with moist paper towels and treated overnight with an intracoelomic injection of $1 \%$ colchicine to enable chromosome preparation. Frogs were euthanized by immersion in a $1 \%$ solution of MS-222, following standard animal care protocols (e.g., ASIH 2004; Animals for Research Act Canada, RRO 1990, Regulation 24). Liver tissue samples were removed from voucher specimens through lateral incisions and preserved in tubes with $95 \%$ non-denatured ethanol. Specimens were fixed in $10 \%$ buffered formalin. Our specimens have been deposited in the Colección de Vertebrados, Universidad de Los Andes, 
Mérida, Venezuela (CVULA). Comparative material (Appendix 1) is housed in the following institutions: Museo de Biología, Universidad del Zulia, Maracaibo, Venezuela (MBLUZ); Museo de la Estación Biológica de Rancho Grande, Maracay, Venezuela (EBRG); Colección de Herpetología del Museo de Biología de la Universidad Central de Venezuela, Caracas, Venezuela (MBUCV); Museo de Historia Natural La Salle, Caracas, Venezuela (MHNLS); Naturalis, formerly the Rijksmuseum van Natuurlijke Historie, Leiden, The Netherlands (RMNH); Division of Amphibians and Reptiles, United States National Museum of Natural History, Washington D.C., USA (USNM); and the Museum of Zoology, University of Michigan, Ann Arbor, Michigan, USA (UMMZ). Abbreviations for natural history collections follow Sabaj Pérez (2014).

\section{Chromosomes}

We examined five specimens of Pristimantis nubisilva sp. nov. (4 males, 1 female) and four specimens of $P$. hoogmoedi sp. nov. (3 males, 1 female) cytogenetically. Mitotic chromosomes were prepared directly from intestine preserved in the field after in vivo colchicine treatment. Meiotic chromosomes were obtained from the testes of male specimens. Techniques for preparation of tissues, hypotonic treatment, and fixation of cells used were described by Schmid et al. (1992). Chromosomes were subjected to conventional staining, including Giemsa staining and C-banding of constitutive heterochromatin, using the protocols of Schmid et al. (1992). Microscopic analyses were conducted on Zeiss Axiophot microscopes.

\section{X-rays}

Digital x-ray images were taken using a Kevex model PXS5-724EA x-ray emitter and a Varian PanScan 4030R detection panel, feeding into Varian image viewing and acquisition software. Depending on the size of the specimen, emission voltage settings of 30 $\mathrm{kV}$ and $45 \mathrm{kV}$ were used and the better image was retained. In order to increase the resolution of images, the distance of the specimens to the x-ray source was adjusted, with smaller specimens brought closer to the emitter. In addition, specimens were placed on a Mylar sheet suspended by a cardboard frame in order to reduce structural interference below specimens to a minimum. Specimens were positioned so that the image rendered would display structures approximating a two-dimensional view as closely as possible. This was particularly important given that images were designed for quantitative as well as qualitative analysis. As a consequence, multiple $\mathrm{x}$-rays needed to be taken of some specimens. Once recorded, digital images were converted into TIF-files and edited using Adobe Photoshop software. In order to standardize and optimize the treatment of digital images, each image was converted to grayscale and adjusted using shadow/highlight settings of shadow (amount 2\%, tonal width $50 \%$, radius 30 pixel), highlight $(50 \%, 60 \%, 30)$, brightness (-19), and mid-tone contrast (-43). In some cases, smaller structures in the $x$-rays (e.g., dentition) required additional, individual adjustments using the contrast slider to improve resolution. X-ray images were used not only to assemble an osteological data set for comparisons; they are also useful to infer the maturity of specimens from the extent of epiphyseal fusion. We were therefore able to ensure that our comparative material consisted only of adults.

\section{Descriptions}

The species diagnoses and descriptions follow BarrioAmorós et al. (2010b), using the terminology for skin consistency of Kaiser et al. (1994a). Comparative data were taken from Walker \& Test (1955), Hardy (2001), Lynch \& La Marca (1993), Kaiser et al. (1994b, 1995), and Rivero $(1961,1964)$. We evaluated specimens of most species, including holotypes, in order to develop a data set as free of procedural differences as possible. Species comparisons were limited to species occurring in the biogeographically contiguous northern mountains of Venezuela (Cordillera de la Costa, Turimiquire massif, Serranía de Paria), including their historic extension into Trinidad (northern range) and Tobago (main ridge). As criteria for sex determination we used the presence of mature testes or vocal slits in males, and oviducts or ova in females, as well as direct observation of calling males or amplexus. Measurements (Table 1) of adult frogs were taken in two ways. External measurements were taken to the nearest $0.1 \mathrm{~mm}$ using digital calipers on preserved specimens. We employed several ratios when comparing the characteristics of our new species to each other and to other species from the region. For example, we considered hand size as small relative to body size when the ratio of $\mathrm{HaL}$ to SVL was $\leq 0.25$. Likewise, we placed hind limbs into three arbitrary categories based on the ratio TIB/SVL (short: < 40\%; moderate: 40-49 \%; long: $\geq 50 \%$ ). Drawings of hands and feet were made directly from image files using a Wacom Intuos 4 tablet (Wacom Technology Corporation, Vancouver, Washington, USA; www. wacom.com). Measurements on digital x-ray images were taken on a MacBook Pro by importing the file into 
Table 1. Measurements taken on specimens of five species from the Península de Paria in northeastern Venezuela. Measurements 1-10 were taken from preserved specimens using digital calipers, whereas the remaining measurements were taken in units of pixels from x-ray images. The latter were only used to calculate length ratios, not to provide absolute measurements. Numbers associated with finger (F) characters denote the finger on which the character was measured.

\begin{tabular}{|c|c|c|c|}
\hline & Measurement name & Abbreviation & Description \\
\hline 1 & snout-vent length & SVL & straight measurement along the body from tip of snout to vent \\
\hline 2 & head width & HW & measured across the head at the angle of the jaws \\
\hline 3 & head length & $\mathrm{HL}$ & measured from the tip of the snout to the occipital condyles evident through the skin \\
\hline 4 & interorbital distance & IOD & distance between proximal edges of eyelids, as visible from the edge of the eye socket \\
\hline 5 & eye diameter & EYE & measured from anterior to posterior edge of eye \\
\hline 6 & eye-to-nostril distance & $\mathrm{E}-\mathrm{N}$ & distance between the anterior edge of the eye to the posterior edge of the nostril \\
\hline 7 & internarial distance & IND & distance measured between the edges of the nares \\
\hline 8 & tympanum diameter & TYM & horizontal tympanum diameter \\
\hline 9 & tibial length & TIB & measured from the outer edge of flexed knee to heel \\
\hline 10 & eye-to-tympanum distance & ET & distance between the anterior edge of the tympanum to the posterior edge of the eye \\
\hline 11 & hand length & HaL & measured from the heel of the hand to the tip of $\mathrm{F}^{3}$ \\
\hline 12 & length of $\mathrm{F}^{1}$ & $\mathrm{~F}^{1} \mathrm{~L}$ & $\begin{array}{l}\text { measured from the proximal end of the metacarpal to the distal end of the terminal } \\
\text { phalanx }\end{array}$ \\
\hline 13 & length of $\mathrm{F}^{2}$ & $F^{2} L$ & as for $\mathrm{F}^{1} \mathrm{~L}$ \\
\hline 14 & length of metacarpal of $\mathrm{F}^{1}$ & $\mathrm{~F}^{1} \mathrm{M}$ & measured from the proximal to the distal end of the metacarpal \\
\hline 15 & length of metacarpal on $\mathrm{F}^{2}$ & $\mathrm{~F}^{2} \mathrm{M}$ & as for $\mathrm{F}^{1} \mathrm{M}$ \\
\hline 16 & length of metacarpal on $\mathrm{F}^{3}$ & $\mathrm{~F}^{3} \mathrm{M}$ & as for $\mathrm{F}^{1} \mathrm{M}$ \\
\hline 17 & length of antepenultimate phalanx on $\mathrm{F}^{3}$ & $\mathrm{~F}^{3} \mathrm{P}^{\mathrm{a}}$ & measured from the proximal to the distal end of the phalanx \\
\hline 18 & length of penultimate phalanx on $\mathrm{F}^{3}$ & $\mathrm{~F}^{3} \mathrm{P}^{\mathrm{p}}$ & as for $\mathrm{F}^{3} \mathrm{P}^{a}$ \\
\hline 19 & length of metacarpal on $\mathrm{F}^{4}$ & $\mathrm{~F}^{4} \mathrm{M}$ & as for $\mathrm{F}^{1} \mathrm{M}$ \\
\hline 20 & skull length & SkL & $\begin{array}{l}\text { measured from the anterior edge of the premaxilla to the posterior end of the occipital } \\
\text { condyle }\end{array}$ \\
\hline 21 & skull width & SkW & measured across the skull at its widest point across the quadratojugal \\
\hline 22 & width of vertebra III & Vert3W & straight horizontal distance between the termini of the transverse processes \\
\hline 23 & width of sacral vertebra & Vertsw & as for Vert3W \\
\hline 24 & femur length & FEM & measured along the entire length of the femur \\
\hline 25 & calcaneum length & CALC & measured along the entire length of the calcaneum \\
\hline 26 & radioulnar length & RU & measured from the elbow joint to the hand articulation \\
\hline 27 & humerus length & HUM & measured from the elbow joint to the shoulder joint \\
\hline 28 & premaxilla to ischium length & Pmlsch & measured from the anterior edge of the premaxilla to the posterior end of the ischium \\
\hline
\end{tabular}

AnalyzingDigitallmages (version 11; Museum of Science, Boston, Massachusetts, USA) and using the line tool to take measurements. Measurements using the latter method were very accurate, producing an error of only $0.5 \%$ in a trial run of 25 measurements over three days. To compare the new species with other species from the region, we selected characteristics easily recognizable in the field or on preserved specimens, but we derived as comprehensive a character list as possible from our own specimen evaluation as well as features listed and images included in the literature (Table 2). Throughout, we refer to fingers $(F)$ and toes $(T)$ with superscripted Arabic numerals (e.g., $\mathrm{T}^{4}=$ the $4^{\text {th }}$ toe). We believe that this minor modification is an overdue update from the use of Roman numerals (e.g., the $4^{\text {th }}$ toe $=$ TIV) and will simplify an understanding of these data. Based on their geographic location and morphological affinities, it is clear that the species described herein reside within the genus Pristimantis, subgenus Pristimantis, as defined by Hedges et al. (2008). It is not possible to ascertain their close phylogenetic relationships without a more comprehensive analysis, and we refer them to species groups with the understanding that the $P$. unistrigatus species group is most likely not monophyletic (Hedges et al. 2008; Hoyos et al. 2014). To coin appropriate common names for these species, we follow the 
suggestion of Barrio-Amorós et al. (2013) to use the term "landfrog," given that these members of Terrarana are direct developers - a more fundamental statement about their biology than their appearance on rainy nights, which is common to almost all frogs. Since we collected several of the paratypes in the same locality as the holotype, we indicate this by the use of the term "paratopotype" (sensu Evenhuis 2009; Maggenti et al. 2005).

\section{SPECIES DESCRIPTIONS}

\section{Pristimantis nubisilva sp. nov. \\ (Images 3A-D, 4A-F, 5A-B, 5A'-B', 7, 20A, 21A)}

urn:Isid:zoobank.org:act:7EDD344F-075B-4278-85E7-25BFEE9AAEA4

English name: Paria Cloud Forest Landfrog

Spanish name: Ranita de Bosque Nublado de Paria

\section{Holotype}

CVULA 7430 (Images 3A, 4F), 4.ix.2001, an adult female from the southern slopes of Cerro Humo, Península de Paria, Estado Sucre, Venezuela $\left(10.7073^{\circ} \mathrm{N}\right.$ \& $\left.62.6284^{\circ} \mathrm{W}\right)$, elevation ca. $750 \mathrm{~m}$, coll. by C. BarrioAmorós, H. Kaiser, and J. Trujillo.

\section{Paratypes}

Eight adult males: CVULA 7423-29, 7431. These have the same collecting data as the holotype and are therefore considered paratopotypes.

\section{Referred specimens}

MHNLS 13347-48, 14463-65, RMNH 28434, 2843638, from Cerro Humo.

\section{Diagnosis}

Pristimantis nubisilva is a small (SVL of males 16.7-21.8, $\bar{x}=19.5 \pm 1.5, n=13$; SVL of only known female $24.5 \mathrm{~mm}$; Table 3) member of the $P$. unistrigatus species group (sensu Hedges et al. 2008) defined by the following characteristics: (1) dorsal skin finely shagreen in life, smooth in preservative, with middorsal raphe (Image $4 A, E$ ) and narrow low dorsolateral folds (e.g., Image $3 \mathrm{~A})$; ventral skin finely areolate on throat and chest, smooth to finely areolate on belly, coarsely areolate in groin region; cranial crests absent; (2) tympanum round, distinct, small (TYM ca. 30\% of EYE; Image 3B), with tympanic annulus; supratympanic fold weak; E-T ca. $2 / 3$ TYM; two small, lighter colored postrictal tubercles present posteriorly to the commissure of the mouth in the straight-line area between the tympanum and the insertion of the arm (e.g., Image 3B,D), their size about that of the narial opening; (3) snout rounded to truncate in dorsal view, rounded in profile; $\mathrm{E}-\mathrm{N}$ ca. $75-80 \%$ of EYE; canthus rostralis distinct, straight in dorsal view, rounded in cross section; loreal region weakly concave; (4) IOD ca. $72 \%$ EYE on average (range 59-94\%); upper eyelid with small tubercles, a single enlarged tubercle present near the center of the eyelid along its margin in most specimens (e.g., Image $3 \mathrm{~A}, \mathrm{C}$ ); (5) choanae small, round; dentigerous processes of the vomers small, triangular, with 4-5 teeth in a linear arrangement on the posterior margin; 10-14 premaxillary teeth; 74-84 maxillary teeth; tongue large, rounded, sub-triangular or quadrangular, not notched to slightly notched, $1 / 2-1 / 3$ free posteriorly; (6) vocal slits and nuptial pads present in males; a single-lobed, round subgular vocal sac present (Image 3C); (7) size of fingers $1 \leq 2=4<3, \mathrm{~F}^{3}$ about twice as long as $\mathrm{F}^{1}$; finger disks expanded, disks on $\mathrm{F}^{2}-\mathrm{F}^{4}$ about $1.5-2$ times wider than digits, in $\mathrm{F}^{1}$ less so (Image 5A, $\mathrm{A}^{\prime}$ ); subarticular tubercles round and raised; deeply bifid palmar tubercle, medial part elliptical and reaching center of palm; lateral part oval and connected to the medial tubercle at the proximal edge of the hand; thenar tubercle large, elongate, extending laterally halfway up the length of $\mathrm{F}^{1}$; few supernumerary palmar tubercles; (8) fingers with narrow and indistinct lateral fringes; (9) several small, indistinct ulnar tubercles; (10) small and indistinct knee and heel tubercles, no enlarged tubercles; inner tarsal fold absent; (11) two metatarsal tubercles, inner metatarsal tubercle elongate, outer tubercle $1 / 4$ size inner and conical (Image $\left.5 B, B^{\prime}\right)$; few supernumerary plantar tubercles; (12) size of toes $1<2<3<5<4$; toe disks oval, disks on $\mathrm{T}^{2}$ and $\mathrm{T}^{4}$ expanded, usually 1.5-2 times wider than digits; lateral fringes almost indistinct; $\mathrm{T}^{5}$ slightly longer than $\mathrm{T}^{3}$, barely reaching the penultimate subarticular tubercle on $\mathrm{T}^{4}$; webbing absent; (13) coloration in life variable, ranging from greenish-pale brown to orange brown (Image 3) with three distinctive patterns (see Variation below); throat white with very light mottling or none, venter darkening posteriorly; concealed surfaces of hind limbs with extensive yellow spotting on a white background, sometimes merging to solid yellow; labial area colored as the dorsum; slightly curved, brown supratympanic stripe, in some specimens forming a continuous line from the canthus rostralis through the eye to the insertion of the forelimb; in preservative grey to pale brown above; ventrally dirty white to dark brown (Image 4D); iris color bronze in life; (14) slight sexual size dimorphism present, 

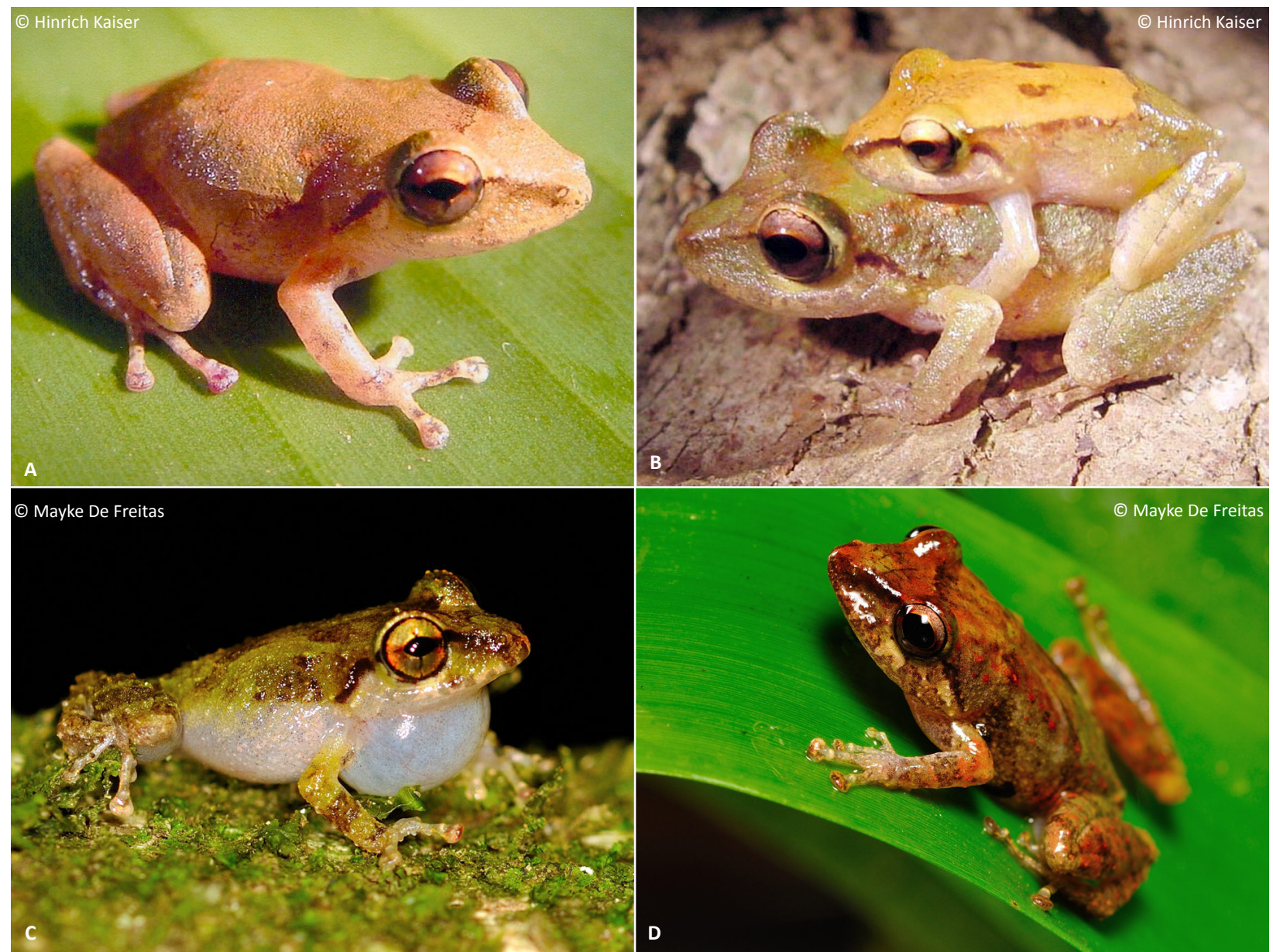

Image 3. Pristimantis nubisilva sp. nov. from the Península de Paria in northeastern Venezuela. (A) Female holotype (CVULA 7430; SVL = $\mathbf{2 4 . 5} \mathrm{mm}$ ) in life. (B) Axillary amplexus between a male and female (specimens not collected). This image clearly shows the greenish brown body coloration in life. (C) Calling male from the Las Melenas area (specimen not collected). (D) Individual from Cerro Humo, showing the red dorsal tuberculation present in some frogs (specimen not collected).

with females ca. $10 \%$ larger than males on average; axillary amplexus (Image 3B); (15) normal karyotype consists of $2 n=36$ telocentric chromosomes (Image 6).

\section{Comparisons}

Pristimantis nubisilva is unique among coastal Cordillera Pristimantis in the following combination of characters: size small, tympanum small (ca. $1 / 3$ of eye diameter) and distinct, with a tympanic annulus; middorsal raphe and weak dorsolateral folds present; discs on fingers and toes widely expanded, oval; yellow coloration in the groin and anterior side of thighs (Image 4).

Pristimantis nubisilva (characters in parentheses) is here compared with species from the Venezuelan Coastal Range, inclusive of its geological extension into Trinidad and Tobago (see Table 2). Pristimantis anotis (Walker \& Test, 1955) is a larger species, with females reaching $47 \mathrm{~mm}$ SVL (only known adult female $24.5 \mathrm{~mm}$ ), and it lacks a tympanum (distinct tympanum present). Pristimantis bicumulus (Peters, 1864) is also larger, with females reaching $37 \mathrm{~mm}$ SVL $(24.5 \mathrm{~mm})$, and has an obscured tympanum (distinct), concave canthus rostralis in dorsal view (straight), oval dentigerous processes of the vomer (triangular), and a single bifid palmar tubercle (deeply bifid palmar tubercle). Pristimantis charlottevillensis (Kaiser et al. 1995), endemic to Tobago, is a much larger species with males reaching $31 \mathrm{~mm} \mathrm{SVL}$ (22mm), $F^{1}>F^{2}\left(F^{1} \leq F^{2}\right)$, an oval tympanum (round), $\mathrm{T}^{5}<\mathrm{T}^{3}\left(\mathrm{~T}^{5}>\mathrm{T}^{3}\right)$, and webbing present between $\mathrm{T}^{4}$ and $\mathrm{T}^{5}$ (no webbing). Pristimantis geminus sp. nov. can be distinguished by its obscured, oval tympanum (distinct, round), ill-defined canthus rostralis (distinct), absence of vocal slits (present), single bifid palmar tubercle (deeply bifid palmar tubercle), and the presence of a pair of protuberant scapular tubercles (absent). 

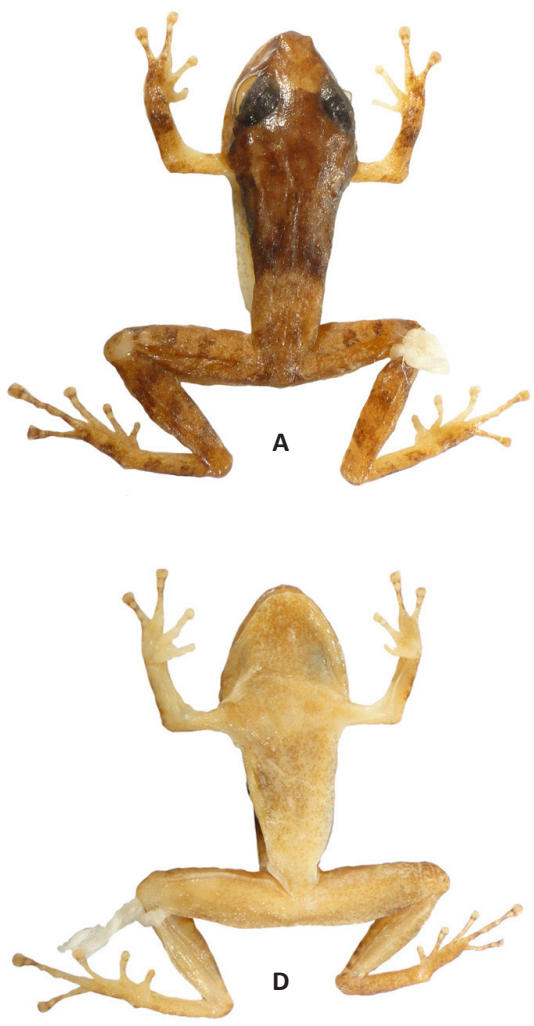
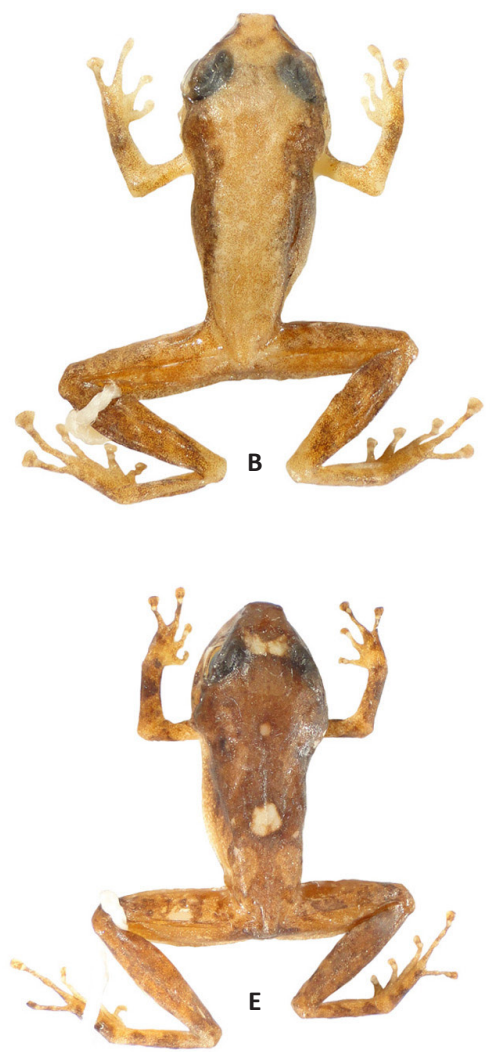
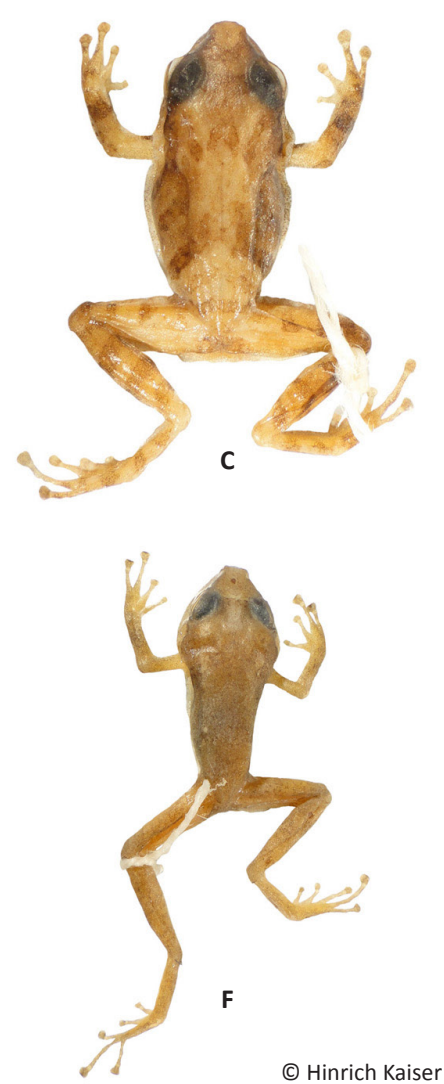

Image 4. Dorsal pattern variation in Pristimantis nubisilva sp. nov., illustrating the shirt-and-trousers phenotype (A; CVULA 7423, male, SVL = 19.3mm), the dorsoconcolor phenotype (B; CVULA 7425, male, SVL $=20.8 \mathrm{~mm}$ ), a combination of shirt-and-trousers and dorsoconcolor pattern (C; CVULA 7427, male, SVL = 19.4mm), a shirt-and-trousers phenotype with a light middorsal blotch (E; CVULA 7424, male, SVL = 18.2mm), and a plain dorsum (F; CVULA 7430, holotype, female, $S V L=24.5 \mathrm{~mm}$ ). The ventral coloration is plain (D; CVULA 7423).

Pristimantis hoogmoedi sp. nov. is a much larger frog, with male SVL up to $36 \mathrm{~mm}(22 \mathrm{~mm})$, eyes red in life (coppery bronze), a concave canthus rostralis in dorsal view (straight), a single bifid palmar tubercle (deeply bifid palmar tubercle), and webbing present between $\mathrm{T}^{4}$ and $\mathrm{T}^{5}$ (no webbing). Pristimantis longicorpus sp. nov. has smooth ventral skin (finely areolate), a truncate snout in dorsal view (rounded), a notched tongue (not notched to slightly notched), a single round palmar tubercle (deeply bifid palmar tubercle), very different hind limb proportions, and significant differences in the size and shape of metatarsal tubercles (Table 2). Pristimantis pariagnomus sp. nov. is much smaller, with adult males reaching a SVL of $13 \mathrm{~mm}(22 \mathrm{~mm})$, has an obscured, oval tympanum (distinct, round), an illdefined canthus rostralis (distinct), one bifid palmar tubercle (deeply bifid palmar tubercle), and webbing between $\mathrm{T}^{4}$ and $\mathrm{T}^{5}$ (no webbing). Pristimantis reticulatus (Walker \& Test, 1955) is a similarly-sized species, with males up to $25 \mathrm{~mm}$ in SVL ( $22 \mathrm{~mm})$, possessing a truncate snout in dorsal view (rounded), a concave canthus rostralis in dorsal view (straight), no vocal slits in males (present), and one bifid palmar tubercle (deeply bifid palmar tubercle). Pristimantis riveroi (Lynch \& La Marca, 1993) is also similarly-sized, with males reaching an SVL of $23 \mathrm{~mm}(22 \mathrm{~mm})$, possessing an obscured, oval tympanum (distinct, round) but no nuptial pads in males (present). Pristimantis rozei (Rivero, 1961) can be differentiated by its concave canthus rostralis in dorsal view (straight), rounded dentigerous processes of the vomers (triangular), and by having $\mathrm{T}^{3}>\mathrm{T}^{5}\left(\mathrm{~T}^{5}>\right.$ $\mathrm{T}^{3}$ ). Pristimantis stenodiscus (Walker \& Test, 1955) is a smaller species, with males up to $17 \mathrm{~mm}$ in SVL $(22 \mathrm{~mm})$, and with discs pointed at tips (rounded), an obscured tympanum (distinct), no vocal slits in males (present), and one bifid palmar tubercle (deeply bifid palmar tubercle). Pristimantis terraebolivaris (Rivero, 1961) is a much larger species, with males reaching $36 \mathrm{~mm}$ in SVL $(22 \mathrm{~mm})$, smooth ventral skin (finely areolate), TYM $2 / 3$ of EYE ( $1 / 3$ of EYE), $F^{1}=F^{2}\left(F^{1}<F^{2}\right)$, and one bifid palmar tubercle (deeply bifid palmar tubercle). Pristimantis turpinorum (Hardy, 2001), a Tobago endemic, is a 

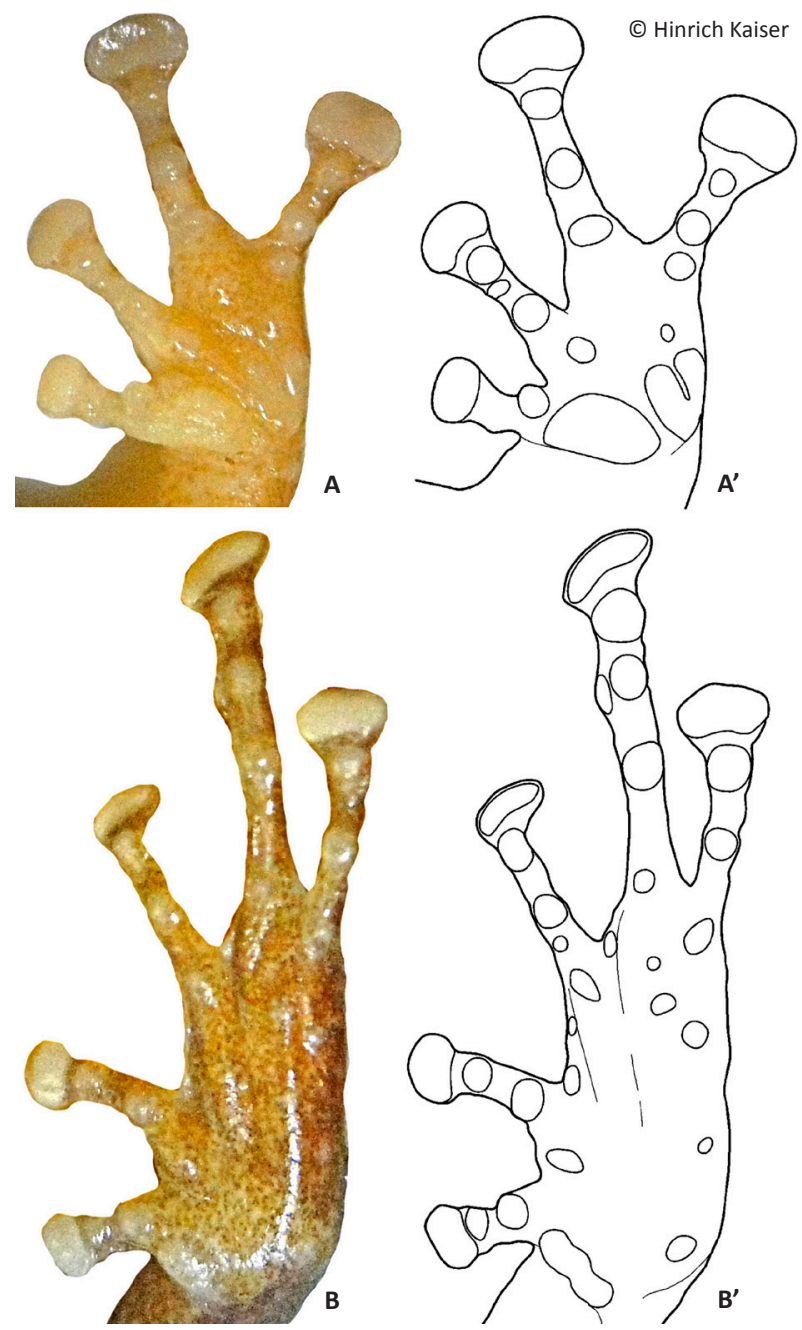

Image 5. Photographs and drawings of hand and foot of Pristimantis nubisilva sp. nov. (A, A') Left hand of MHNLS 14465. (B, B') Left foot of MHNLS 13348.

similarly-sized species, with males reaching $19 \mathrm{~mm}$ in SVL $(22 \mathrm{~mm})$, smooth dorsal skin (finely shagreen), obscured tympanum (distinct), truncate snout in dorsal view (round), one bifid palmar tubercle (deeply bifid palmar tubercle), and no tarsal tubercles (present). Pristimantis turumiquirensis (Rivero, 1961), found only in the Turumiquire massif, is a much larger species, with females reaching $46 \mathrm{~mm}$ in SVL (the only female is $24.5 \mathrm{~mm}$ in SVL), concave canthus rostralis in dorsal view (straight), and by having $\mathrm{T}^{3}$ longer than $\mathrm{T}^{5}\left(\mathrm{~T}^{5}>\mathrm{T}^{3}\right)$. Lastly, P. urichi (Boettger, 1894) is a similarly-sized species, with males reaching $23 \mathrm{~mm}$ in SVL $(22 \mathrm{~mm})$, an obscured tympanum (distinct), concave canthus rostralis in dorsal view (straight), a pair of scapular tubercles (absent), and a blue upper iris in life (bronze).

\section{Description of the holotype}

An adult female, SVL $=24.5 \mathrm{~mm}$. Body slender; head wider than body, $16 \%$ wider than long, $\mathrm{HL} 42 \%$ of SVL, HW $42 \%$ of SVL. Snout rounded in dorsal view, rounded in profile; E-N 79\% of EYE; nostrils slightly protuberant, directed dorsolaterally; canthus rostralis distinct, straight in dorsal view, rounded in cross section; loreal region weakly concave. Upper eyelid with small, rounded tubercles, none subconical or conical. Cranial crests absent. Tympanum distinct, $28 \%$ of EYE, surrounded by a tympanic annulus, only apparent in its infero-anterior section, with a short supratympanic fold covering a small portion of its posterodorsal section. Choanae small, rounded, not concealed by palatal shelf of maxillary arch; vomerine dentigerous processes small, triangular, slightly oblique, widely separated, positioned posteriorly and medially to choanae, bearing five teeth each. Tongue large, sub-triangular, not notched posteriorly, posterior half free.

Dorsal skin finely granular in life, smooth in preservative; some small tubercles in the interorbital and frontonasal area; supra- and post-tympanic area bearing small but protuberant tubercles; one larger tubercle at the posterior end of the indistinct occipital ridges; middorsal raphe present but indistinct; dorsolateral folds on the anterior half of the body, narrow, low and

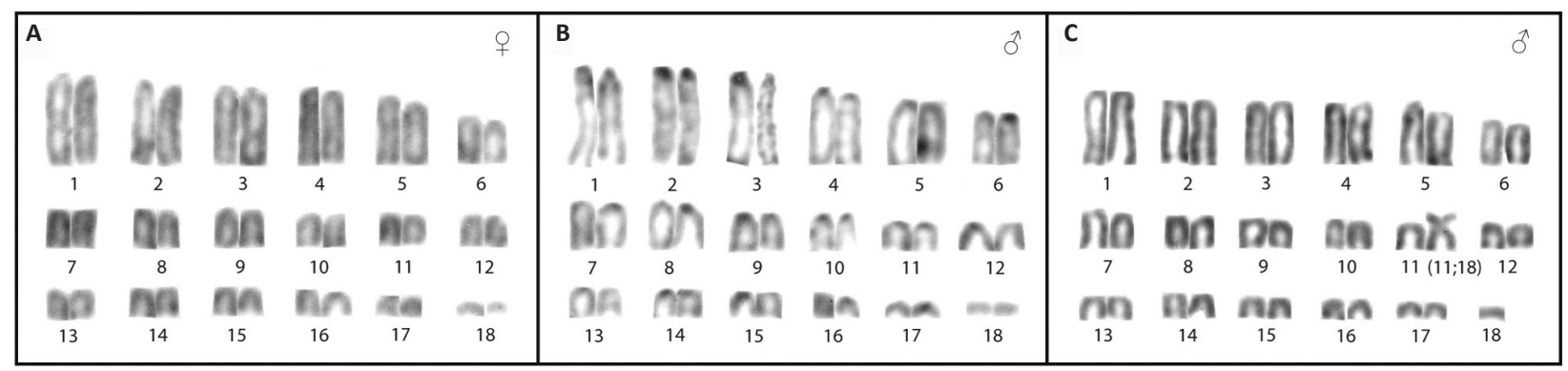

Image 6. Conventionally Giemsa-stained mitotic chromosomes of (A) female and (B, C) male Pristimantis nubisilva sp. nov. The karyotype of the male depicted in (C) shows a heterozygous centric fusion between chromosomes 11 and 18, resulting in a submetacentric fusion chromosome $11 ; 18$. 


\begin{tabular}{|c|c|c|c|c|c|c|c|c|c|c|c|c|}
\hline a & $\stackrel{\stackrel{N}{N}}{\sim}$ & $z$ & 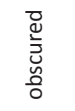 & 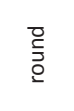 & $\stackrel{n}{\rightarrow}$ & $\begin{array}{l}\text { 苾 } \\
\text { 幽 }\end{array}$ & 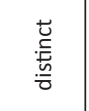 & 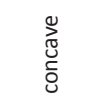 & 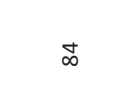 & $\overline{\mathrm{g}}$ & $\begin{array}{l}\overline{0} \\
\overline{0} \\
\end{array}$ & 京离 \\
\hline$\circ$ & go & z & 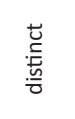 & 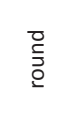 & $\stackrel{\sim}{\exists}$ & $\begin{array}{l}\stackrel{\check{\varpi}}{\varpi} \\
\stackrel{3}{3}\end{array}$ & 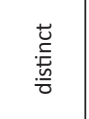 & 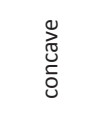 & R & 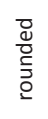 & 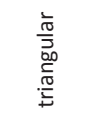 & 总离 \\
\hline$z$ & ने & z & 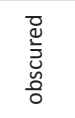 & $\begin{array}{l}\overline{0} \\
\stackrel{\underline{5}}{0} \\
\underline{0}\end{array}$ & $\stackrel{m}{\rightarrow}$ & 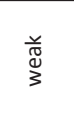 & 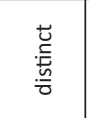 & 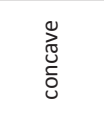 & ה & 뭉 & 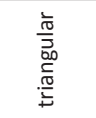 & $\begin{array}{l}\varphi^{0} \stackrel{\bar{\varpi}}{\varpi ّ} \\
\omega\end{array}$ \\
\hline$\Sigma$ & 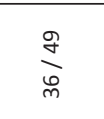 & z & $\begin{array}{l}\text { 蒙 } \\
\frac{\underline{\underline{b}}}{\sigma}\end{array}$ & $\begin{array}{l}\overline{0} \\
\text { 홈 }\end{array}$ & $\stackrel{m}{\sim}$ & 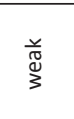 & 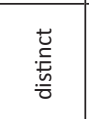 & 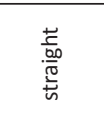 & g & 뭉 & 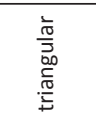 & 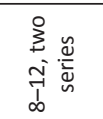 \\
\hline$\lrcorner$ & 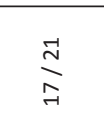 & $z$ & 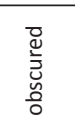 & 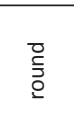 & $\stackrel{\circ}{\underset{7}{*}}$ & 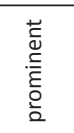 & $\begin{array}{l}\stackrel{\vec{E}}{\vec{E}} \\
\frac{\underline{\underline{m}}}{\sigma}\end{array}$ & $\begin{array}{l}0 \\
\stackrel{0}{0} \\
\overline{0} \\
0\end{array}$ & $\stackrel{\infty}{n}$ & 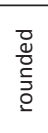 & 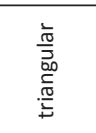 & 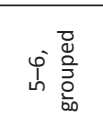 \\
\hline$\simeq$ & $\stackrel{0}{i}$ & & & & $\stackrel{m}{\rightarrow}$ & & 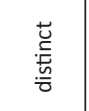 & 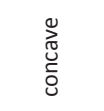 & $\stackrel{\infty}{\circ}$ & & 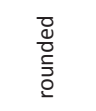 & 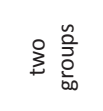 \\
\hline- & 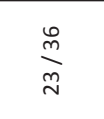 & z & 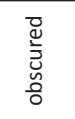 & $\overline{\mathrm{g}}$ & 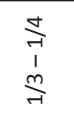 & 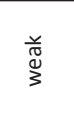 & 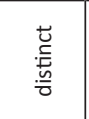 & 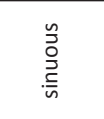 & & 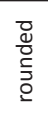 & 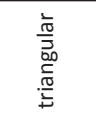 & 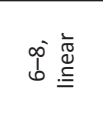 \\
\hline- & 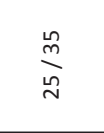 & z & 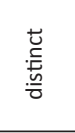 & $\begin{array}{l}\overline{0} \\
\text { 홈 }\end{array}$ & 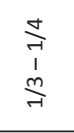 & 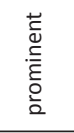 & 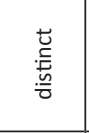 & 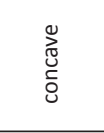 & $\stackrel{\infty}{\stackrel{\infty}{2}}$ & 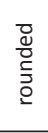 & 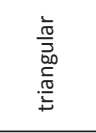 & 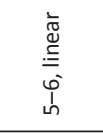 \\
\hline$I$ & $\stackrel{\sim}{m}$ & z & 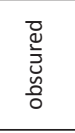 & 产 & $\hat{\mathrm{N}}$ & 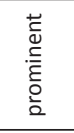 & 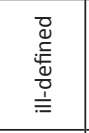 & 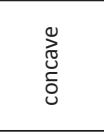 & $\overrightarrow{1}$ & 훙 & 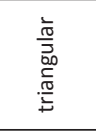 & 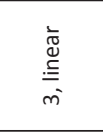 \\
\hline 0 & $\stackrel{\stackrel{n}{N}}{N}$ & $>$ & 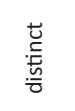 & 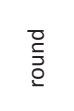 & $\stackrel{m}{\rightarrow}$ & 总 & 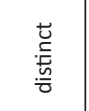 & 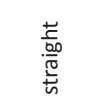 & $\approx$ & 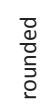 & 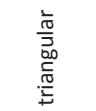 & 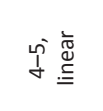 \\
\hline 4 & $\stackrel{\sim}{\sim}$ & $z$ & 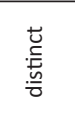 & 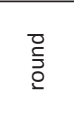 & $\stackrel{m}{\vec{r}}$ & 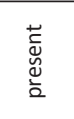 & 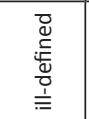 & 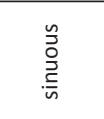 & g & 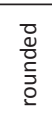 & 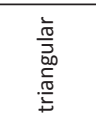 & 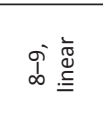 \\
\hline س & $\underset{\substack{n \\
0}}{\vec{n}}$ & z & 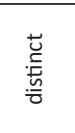 & 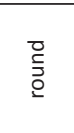 & $\stackrel{A}{A}$ & 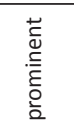 & 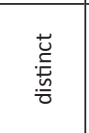 & 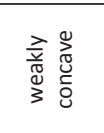 & 8 & $\begin{array}{l}\frac{0}{0} \\
0 \\
0\end{array}$ & 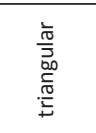 & 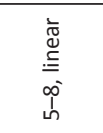 \\
\hline 0 & $\stackrel{\sim}{i}$ & $>$ & 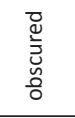 & $\overline{\mathrm{g}}$ & $\stackrel{0}{t}$ & $\begin{array}{l}\text { 苾 } \\
\text { 岕 }\end{array}$ & 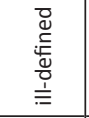 & 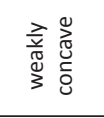 & 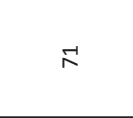 & 뭉 & 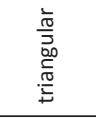 & 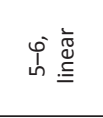 \\
\hline$u$ & $\stackrel{\infty}{\stackrel{\infty}{-m}}$ & z & 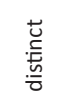 & $\overline{\mathrm{g}}$ & $\stackrel{ \pm}{*}$ & 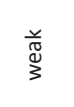 & 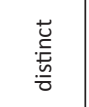 & 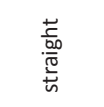 & $\infty$ & 흠 & 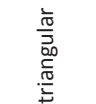 & 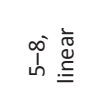 \\
\hline$\infty$ & 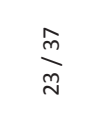 & $z$ & 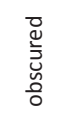 & 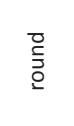 & $\stackrel{n}{r}$ & 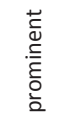 & 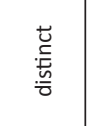 & 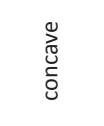 & R & 믕 & $\overline{\mathrm{g}}$ & 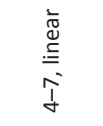 \\
\hline$\varangle$ & $\stackrel{\text { f }}{\text { in }}$ & $>$ & 䒓 & $\stackrel{\pi}{\varepsilon}$ & $\stackrel{\pi}{E}$ & $\stackrel{\pi}{\Xi}$ & 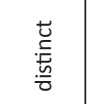 & 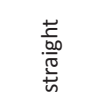 & 8 & 응 & 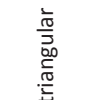 & 守离 \\
\hline 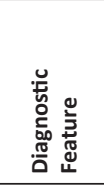 & 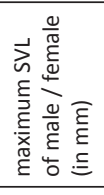 & 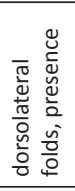 & 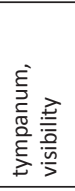 & 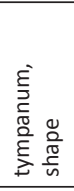 & 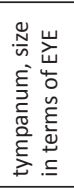 & 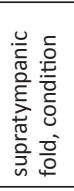 & 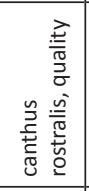 & 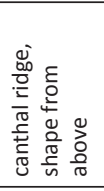 & 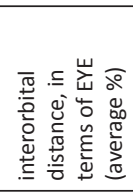 & $\begin{array}{l}\frac{0}{0} \\
\frac{0}{5} \\
\frac{0}{0} \\
\frac{0}{0} \\
\frac{0}{0} \\
\frac{0}{c} \\
\end{array}$ & 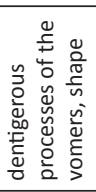 & 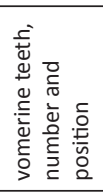 \\
\hline 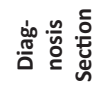 & $\neg$ & & N & & & & $m$ & & $\sigma$ & n & & \\
\hline
\end{tabular}




\begin{tabular}{|c|c|c|c|c|c|c|c|c|c|c|c|c|c|}
\hline a & & & $>$ & $z$ & 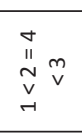 & 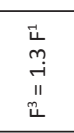 & 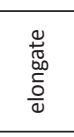 & $z$ & 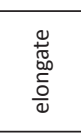 & 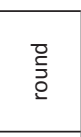 & $\stackrel{0}{\rightarrow}$ & $\begin{array}{l}v \\
v \\
v \\
v \\
v \\
v \\
v\end{array}$ & 旁 \\
\hline ० & & & c. & c. & $\underset{\underset{\sim}{v}}{\underset{\sim}{v}} \underset{v}{v}$ & 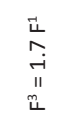 & 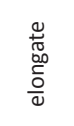 & $>$ & 总 & 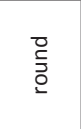 & $\stackrel{\stackrel{n}{t}}{\rightarrow}$ & $\begin{array}{l}\stackrel{v}{v} \\
\stackrel{v}{v} \\
\underset{v}{v} \\
v \\
v\end{array}$ & 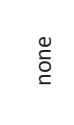 \\
\hline$z$ & $\underset{7}{\stackrel{1}{a}}$ & $\begin{array}{l}0 \\
\vdots \\
\grave{0}\end{array}$ & $>$ & z & $\begin{array}{l}\underset{v}{v} \\
\underset{v}{\sim} \\
\underset{v}{v}\end{array}$ & 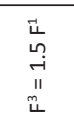 & 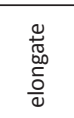 & $z$ & $\overline{\mathrm{g}}$ & 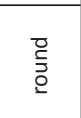 & $\stackrel{\Delta}{-}$ & $\begin{array}{l}v \\
v \\
v \\
v \\
v \\
v \\
v \\
v\end{array}$ & $\stackrel{n}{1}$ \\
\hline$\Sigma$ & & & $>$ & z & $\begin{array}{l}\underset{\sim}{v} \\
\underset{\sim}{N} \\
\vec{H}\end{array}$ & 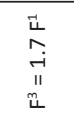 & 产 & $>$ & $\overline{\mathrm{g}}$ & 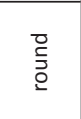 & 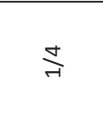 & $\begin{array}{l}v \\
v \\
v \\
v \\
v \\
v \\
v \\
v \\
v\end{array}$ & 䒕 \\
\hline$\lrcorner$ & 이 & $\begin{array}{l}\hat{1} \\
\hat{d} \\
0\end{array}$ & z & z & 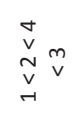 & $\begin{array}{l}\vec{山} \\
\stackrel{\text { II }}{\text { II }}\end{array}$ & 嵒 & $z$ & 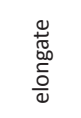 & $\begin{array}{l}\overline{0} \\
\text { 旁 }\end{array}$ & $\stackrel{0}{=}$ & $\begin{array}{l}v \\
\stackrel{v}{n} \\
\| \\
\stackrel{v}{v} \\
v \\
v \\
v \\
m\end{array}$ & 旁 \\
\hline$\simeq$ & & & & & $\begin{array}{l}\underset{+}{v} \\
\underset{v}{\sim} \\
\underset{-1}{v}\end{array}$ & 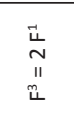 & & & & & & $\begin{array}{l}v \\
v \\
v \\
v \\
v \\
v \\
v \\
v\end{array}$ & 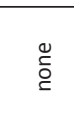 \\
\hline - & & & $>$ & $z$ & & & $\overline{\frac{\mathrm{g}}{\partial}}$ & $>$ & $\overline{\frac{\mathrm{g}}{\partial}}$ & $\begin{array}{l}\text { o } \\
\text { 音 }\end{array}$ & $\stackrel{m}{\vec{H}}$ & & \\
\hline- & & & $z$ & $z$ & $\underset{\sim}{\stackrel{+}{v}} \underset{\sim}{N} \stackrel{m}{v}$ & 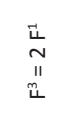 & 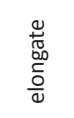 & z & 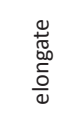 & $\begin{array}{l}\bar{o} \\
\text { ō } \\
\underline{\underline{z}}\end{array}$ & $\underset{I}{ \pm}$ & 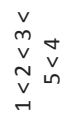 & 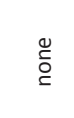 \\
\hline$I$ & $\infty$ & $\begin{array}{l}\text { ơ } \\
\text { J }\end{array}$ & z & $z$ & 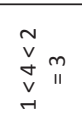 & 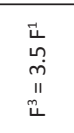 & 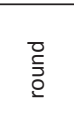 & $z$ & $\overline{\mathrm{g}}$ & 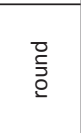 & $\vec{F}$ & 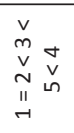 & $\stackrel{r}{i}_{i}^{2}$ \\
\hline ט & \begin{tabular}{l} 
t \\
\multirow{1}{1}{}
\end{tabular} & $\begin{array}{l}\mathbf{1} \\
0 \\
\infty \\
\infty \\
0\end{array}$ & $>$ & $>$ & 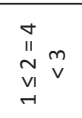 & 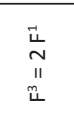 & 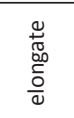 & $>$ & 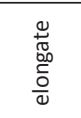 & 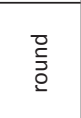 & $\underset{\exists}{ \pm}$ & $\begin{array}{l}\stackrel{v}{v} \\
\tilde{m} \\
\stackrel{v}{v} \\
v \\
v \\
v\end{array}$ & 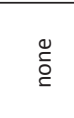 \\
\hline 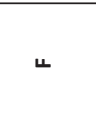 & $\overrightarrow{\underline{d}}$ & I & $z$ & z & 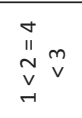 & $\begin{array}{l}\vec{u} \\
\stackrel{\vec{u}}{n} \\
\rightarrow \\
\| 1 \\
\tilde{u}\end{array}$ & $\begin{array}{l}\bar{c} \\
\stackrel{\bar{z}}{\underline{b}}\end{array}$ & z & $\overline{\mathrm{g}}$ & $\overline{\mathrm{g}}$ & $\stackrel{\sim}{\sim}$ & $\begin{array}{l}\vec{v} \\
m \\
v \\
v \\
v \\
v \\
v\end{array}$ & 号 \\
\hline س & 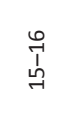 & $\begin{array}{l}\infty \\
0 \\
\frac{1}{0} \\
0\end{array}$ & $z$ & z & $\begin{array}{l}\underset{v}{v} \\
\underset{\sim}{v} \\
\vec{v}\end{array}$ & 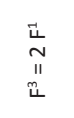 & 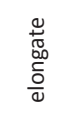 & $>$ & 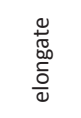 & $\overline{\frac{\mathrm{g}}{\partial}}$ & $\stackrel{\infty}{\rightarrow}$ & 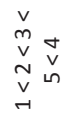 & 焉空 \\
\hline 0 & $\underset{\sim}{\tilde{I}}$ & 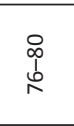 & $z$ & $z$ & $\begin{array}{l}\underset{v}{v} \\
\underset{v}{v} \\
\underset{-1}{v}\end{array}$ & 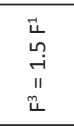 & $\overline{\frac{\mathrm{g}}{\mathrm{O}}}$ & $>$ & $\begin{array}{l}\frac{y}{5} \\
\text { o. } \\
\frac{0}{\alpha}\end{array}$ & $\begin{array}{l}\bar{o} \\
\underline{\underline{z}}\end{array}$ & $\stackrel{0}{\rightarrow}$ & 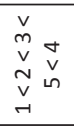 & $\stackrel{n}{T}$ \\
\hline$u$ & & & $>$ & $z$ & 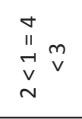 & 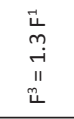 & 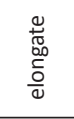 & $>$ & 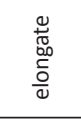 & $\begin{array}{l}\overline{0} \\
\stackrel{\bar{z}}{0} \\
\underline{\underline{0}}\end{array}$ & $\stackrel{n}{*}$ & $\begin{array}{l}v \\
\stackrel{v}{v} \\
v \\
\underset{v}{v} \\
v \\
m\end{array}$ & $\stackrel{n}{T}$ \\
\hline$\infty$ & & & $z$ & $z$ & & & $\overline{\frac{\mathrm{m}}{\partial}}$ & $>$ & 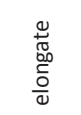 & $\begin{array}{l}\overline{0} \\
\overline{\bar{z}} \\
\underline{0}\end{array}$ & $\underset{\nexists}{+}$ & & $\stackrel{\text { }}{\stackrel{0}{\check{c}}}$ \\
\hline$\varangle$ & $\approx$ & $\begin{array}{l}0 \\
\$ \\
0 \\
6\end{array}$ & $\cdots$ & c. & 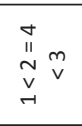 & 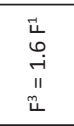 & 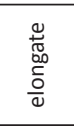 & $>$ & 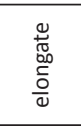 & 疍 & $\stackrel{n}{r}$ & $\begin{array}{l}v \\
\tilde{m} \\
v \\
v \\
v \\
v \\
v \\
v\end{array}$ & $\stackrel{n}{T}$ \\
\hline 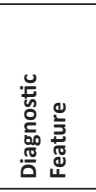 & 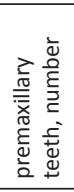 & 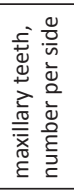 & 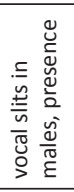 & 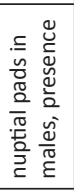 & 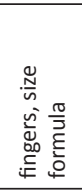 & 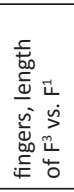 & 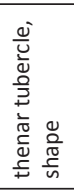 & 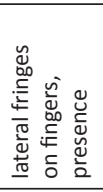 & 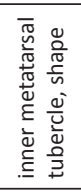 & 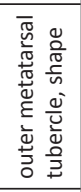 & 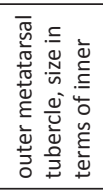 & 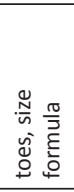 & 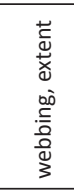 \\
\hline 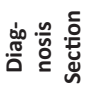 & & & 0 & & r & & & $\infty$ & $F$ & & & $\approx$ & \\
\hline
\end{tabular}


indistinct (affected by preservation). Throat and chest smooth, belly finely granular; ventral surfaces of thighs shagreen. Ulnar and tarsal tubercles low, enlarged heel tubercles absent.

Hand small, HaL $26 \%$ of SVL. Relative lengths of adpressed fingers $1<2=4<3$; when adpressed, $F^{1}$ tip barely reaches disk on $\mathrm{F}^{2}$. Finger disks broader than long, disk on $\mathrm{F}^{3} 1.5$ times wider than adjacent phalanx; disk on $\mathrm{F}^{4}$ nearly twice as wide than adjacent phalanx; disks on $\mathrm{F}^{1}$ and $\mathrm{F}^{2}$ oval, on $\mathrm{F}^{3}$ and $\mathrm{F}^{4}$ more quadrangular; disk on $F^{1} 1.4$ times width of adjacent phalanx. Fingers bearing narrow lateral fringes; webbing absent. Small deeply bifid palmar tubercle and large ovoid thenar tubercle low, distinct; subarticular tubercles protuberant, single, round; few protuberant supernumerary tubercles.

Hind limbs long; TIB 50\% of SVL (Table 4). Relative lengths of adpressed toes $1<2<3<5<4$. $\mathrm{T}^{5}$ Ionger than $\mathrm{T}^{3}$; tip of disk of $\mathrm{T}^{3}$ surpassing antepenultimate subarticular tubercle of $\mathrm{T}^{4}$; tip of $\mathrm{T}^{5}$ reaching penultimate subarticular tubercle on $\mathrm{T}^{4}$; disk on $\mathrm{T}^{4}$ slightly smaller than disk on $\mathrm{F}^{3}$. Toes with almost indistinct lateral fringes, not webbed. Disks distinctly wider than long, wider than phalanges, transversely oval, except on $\mathrm{T}^{1}$ and $\mathrm{T}^{2}$, where they are more rounded. Inner metatarsal tubercle elliptical, distinct; outer small, slightly protuberant, nearly round; subarticular tubercles slightly protuberant, single, round.

\section{Measurements of holotype (in $\mathrm{mm}$ )}

SVL: 24.5; TIB: 12.4; HL: 10.4; HW: 10.2; IND: 2.2; E-N: 3.1; EYE: 3.9; IOD: 3.0; TYM: 1.1; F'L: 3.0; F²L: 3.7.

\section{Color in life}

Dorsum light brown with a soft green sheen, patternless, with a narrow dark interorbital bar and some small, faint dark spots near the snout. Flanks similarly colored as the dorsal area. Limbs without bars; some dark spots on the tibia. Palms of hands and feet dirty white. Diffuse yellow spotting in the hidden areas of groin and; smaller yellow spots exist on the posterior side of the thighs. Ventrally dirty white, the off-white coloration caused by small melanophores.

\section{Color in preservative}

Dorsum pale greyish brown, without any pattern, only a faint and narrow interorbital bar and a small oval black spot on the frontonasal region. Flanks little darker than dorsal area. Arms and hind limbs with no traces of bars; two small black rounded spots on right shank. Hands and feet grey. On the groin and anterior side of thighs there are larger creamy spots (yellow in life); on the posterior side of the thighs there are much smaller, cream-colored spots. Ventrally dirty white; under magnification the whole surface, including throat, chest, belly, undersides of arms and hind limbs, palms and soles, is white with a consistent profusion of melanophores.

\section{Variation}

Males are smaller than the female holotype (Table 3 ). One male (CVULA 7425, Image 4B), is consistent with all characters mentioned for the female holotype, except for the presence of vocal slits, vocal sac, and glandular white nuptial pads (almost indistinct). Hidden surfaces of the hind limbs are patterned in yellow (Image 7).

In terms of coloration, we define three distinct color patterns. Pattern 1 ('plain'; Image 4F) is defined as a plain coloration, seen in the holotype (CVULA 7430), which includes $22.2 \%$ of the type series (holotype described above). It is also seen in CVULA 7428, a specimen of plain dark brown dorsal coloration without any pattern. That specimen is white ventrally, with a moderate profusion of melanophores that are heavier on the chin. Pattern 2 is the 'dorsoconcolor' pattern of Lynch \& La Marca (1993), consisting of a plain, pale brown dorsum with dark brown lateral coloration (Image 4B,C). This pattern is seen in two specimens of the type series (CVULA 7425, 7427), 22.2\% of the type series, and also in MHNLS 13348. Pattern 3 ('shirt and trousers'; Image $4 \mathrm{~A}$ ) is composed of a darker brown color on the anterior of the dorsum, reaching the sacral region (the 'shirt'), and a lighter brown color on the post-sacral portion

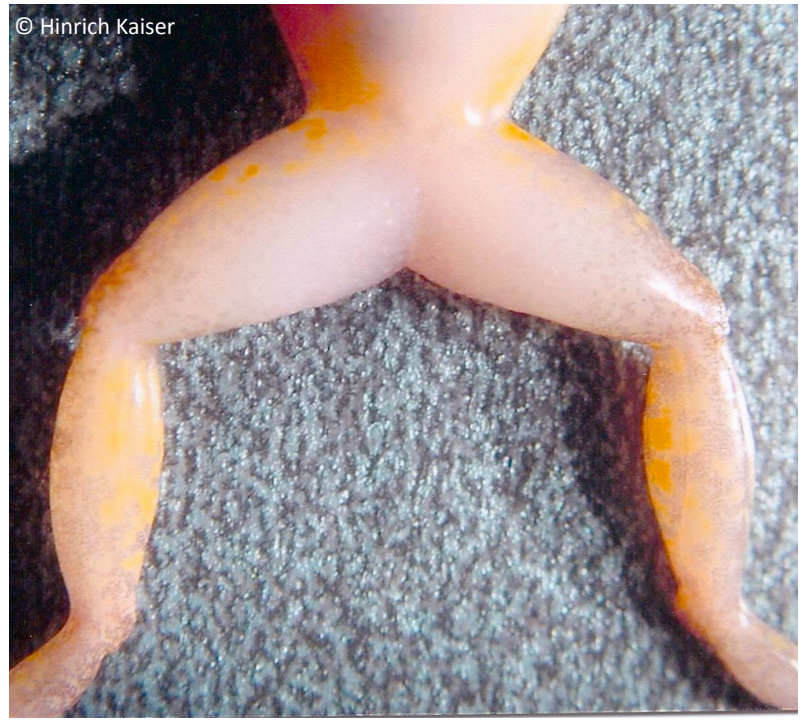

Image 7. Ventral coloration of the hind limbs of Pristimantis nubisilva sp. nov. (CVULA 7425), illustrating the extensive yellow spotting. 
of the dorsum and the legs (the 'trousers'). This is the most common pattern found in the type series (CVULA $7423-24,7426,7428-28,7431)$ and also among the referred specimens (MHNLS 13347, 14464-65). There are two additional variants to the dorsal coloration, one in which a set of pale blotches is added to the 'shirt and trousers' pattern (e.g., CVULA 7424, MHNLS 14464; Image $4 \mathrm{E}$ ), and the other with the dorsoconcolor pattern added to the 'shirt and trousers' pattern (e.g., CVULA 7427; Image 4C).

The dorsal ground color in life is quite variable, but it invariably consists of brown tones with different green hues. In some cases, the color is overall relatively light brown (e.g., Image 3A,B; both photographs taken just after capture) but it can range into the deeper green and brown tones (e.g., Image 3C,D, respectively; both photographed while active in the wild). The 'shirt and trouser' pattern may be differentiated by color, not only by a grade of brown, as shown in the yellow 'shirt' displayed by the amplexed male (Image 3B). Color presentation is likely dependent to a significant degree by environmental and physiological influences, and may produce some fairly bright components, such as small red tubercles to outline the edges of the dorsolateral folds in what could be the dorsoconcolor pattern (Image 3D).

\section{Chromosomes}

Pristimantis nubisilva was listed as "sp. n. L." by Schmid et al. (2010). The single female and three male specimens examined exhibit a karyotype with 36 telocentric chromosomes (Image 6A,B), whereas another male presents a karyotype of 35 telocentric chromosomes and a centric fusion between chromosomes 11 and 18 in heterozygous condition (Image 6C). The fusion chromosome $(11 ; 18)$ is a midsized submetacentric. Analysis of additional male individuals will be necessary to determine if this fusion chromosome, designated as cfu $(11 ; 18)$, represents a $\mathrm{Y}$-autosomal fusion that characterizes the karyotype of some male individuals in this population. Such Y-autosomal fusions have been described in two other species in the Terrarana, Strabomantis biporcatus (Schmid et al. 1992, 2002) and Pristimantis riveroi (Schmid et al. 2003). Three other Pristimantis species and one Eleutherodactylus species have confirmed Y-autosomal fusions, and in one Pristimantis species and five Eleutherodactylus species they are suspected to exist (Schmid et al. 2010). In all these members of the Terrarana, males carrying the Y-autosomal fusion chromosome co-exist with males possessing the original karyotype and lacking the centric fusion chromosome. It should be emphasized that a karyotype consisting of 36 exclusively telocentric chromosomes $(2 n=36, \mathrm{FN}=$ $36)$, as shown by the single female and three males of Pristimantis nubisilva (Image $6 \mathrm{~A}, \mathrm{~B}$ ) is characteristic of many Pristimantis species and is considered to represent the ancestral condition in the taxon Terrarana (Schmid et al. 2010).

\section{Natural History}

Males establish calling sites on a variety of substrates and at a variety of heights (within $3 \mathrm{~m}$ of the ground). These included tree trunks, banana trunks, roots, and branches. We observed individuals in a freshly cut plantation on the debris, on the vegetation surrounding a building, as well as deep in the adjacent forest. Males space calls widely, with 4-5 males able to synchronize calls. We always observed males calling facing head-up. The call is a single, short click in middle frequency. Males appear greenish at night, with only very little dorsal patterning observed. The groin and the anterior hidden surfaces of the thigh are spotted yellow to varying degrees (Image 4). Calling activity was mainly restricted to the time between dusk and $21: 00 \mathrm{hr}$, with hardly any calls heard after 21:00hr. Two pairs of frogs were observed in amplexus and photographed (Image 3B). One pair was collected and remained in amplexus even while they were set up for photography. The species is sympatric with $P$. hoogmoedi sp. nov. and $P$. longicorpus sp. nov. along the edges of some forest streams.

\section{Distribution}

Only known from the southern slope of Cerro Humo, Península de Paria, possibly extended through the cloud forests from 650-1200 m

\section{Etymology}

The species name nubisilva is a composite noun (from the Latin words "nubes" = cloud, and "silva" = forest) used in apposition. It refers to the cloud forest habitat where the species lives. 


\section{Pristimantis hoogmoedi sp. nov.}

(Images 8A-B, 9A-E, 10A-B, 10A'-B', 20B, 21B)

urn:Isid:zoobank.org:act:416D1D0F-3E0B-4205-970C-A4EA5A933A6F

English name: Hoogmoed's Red-Eyed Landfrog Spanish name: Ranita de Ojos Rojos de Hoogmoed

\section{Holotype}

CVULA 7433 (Image 9A,E), 4.ix.2001, an adult female from the southern slopes of Cerro Humo $\left(10.7073^{\circ} \mathrm{N} \&\right.$ $62.6284^{\circ} \mathrm{W}$ ), elevation $750 \mathrm{~m}$, Estado Sucre, Venezuela, coll. by C. Barrio-Amorós and $\mathrm{H}$. Kaiser.

\section{Paratypes}

Four adult females (CVULA 7434-36, 7438) and four males (CVULA 7437, 7439-40, 7442). These have the same locality data as the holotype and are therefore considered paratopotypes.

\section{Referred Specimens}

MHNLS 13345-6, 14457, 14460-61, RMNH 28435, 28434, 28475, from Cerro Humo.

\section{Diagnosis}

Pristimantis hoogmoedi sp. nov. is a relatively large (SVL of six males: 27.9-35.5, $\bar{x}=32.1 \pm 2.8$; SVL of eight females 33.2-51.0, $\bar{x}=40.7 \pm 5.6 \mathrm{~mm}$; Table

\footnotetext{
* Pristimantis hoogmoedi sp. nov. is most similar in overall body morphology to $P$. anotis from the area near the Rancho Grande research station in the Cordillera de la Costa, near Maracay, Estado Aragua, Venezuela. While there is some superficial similarity to species in both the $P$. conspicillatus and the likely polyphyletic $P$. unistrigatus group, we treat $P$. anotis and $P$. hoogmoedi as species incertae sedis within Pristimantis.
}

3) frog, currently not assignable to any known species group of Pristimantis*, characterized by: (1) dorsal skin smooth to finely shagreen, without middorsal raphe or folds, but in some specimens with a low W-shaped occipital ridge (e.g., Image 8A); ventral skin smooth on throat and chest, finely areolate on belly, coarsely areolate in groin; dorsolateral folds and cranial crests absent; (2) tympanum round, distinct, small (TYM about $1 / 4$ of EYE), with tympanic annulus (e.g., Image $8 B$ ); supratympanic fold present and prominent, obscuring the upper third of the tympanum; E-T slightly less than TYM; two very small, lighter colored postrictal tubercles present posteriorly to the commissure of the mouth in the straight-line area between the tympanum and the insertion of the arm, their size less than half that of the narial opening; (3) snout subacuminate to truncate in dorsal view, subacuminate in profile; EYE ca. $30 \%$ greater than $\mathrm{E}-\mathrm{N}$; canthus rostralis distinct, angled to rounded in dorsal view (e.g., Image 8B); canthus rostralis weakly concave, outlined laterally by a dark line; loreal region concave; (4) IOD ca. $2 / 3$ EYE; upper eyelid smooth, with (in life) or without (in some preserved specimens) small tubercles; one prominent supraocular tubercle present on posterior half of eyelid; (5) choanae small, ovoid; dentigerous processes of the vomers small, triangular, bearing 5-8 teeth in a single row along their posterior margin; 10-15 premaxillary teeth; 52-58 maxillary teeth; tongue large, round, notched medially, $1 / 3$ free posteriorly; (6) males lacking vocal slits and nuptial pads; with swollen thumbs; (7) Size of fingers $1<2<4<$ $3, F^{3}$ about twice as long as $F^{1}$ (Image $10 A, A^{\prime}$ ); $F^{1}$ swollen in males; disks on $\mathrm{F}^{2-4}$ rounded, about twice as wide as digit, disk of $F^{1}$ about 1.5 times as wide (Image 10A, $A^{\prime}$ ); subarticular tubercle on $\mathrm{F}^{1-3}$ rounded and raised, on $\mathrm{F}^{4}$
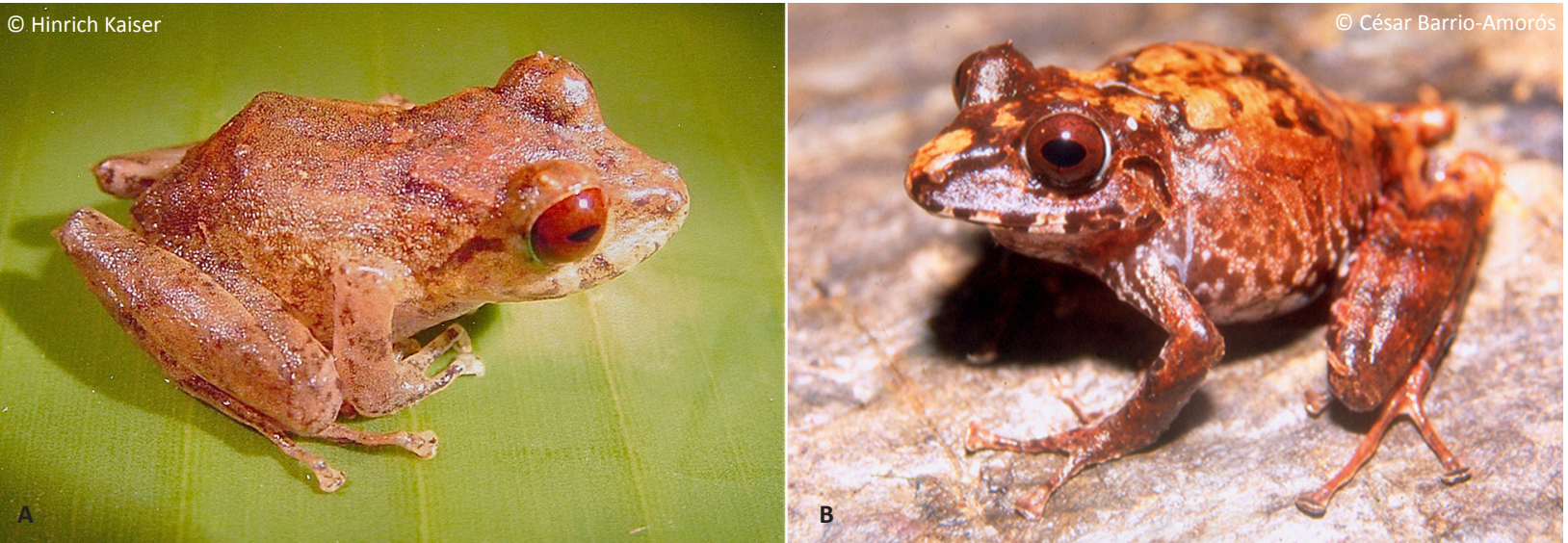

Image 8. Specimens of Pristimantis hoogmoedi sp. nov. from the Península de Paria in northeastern Venezuela, photographed in life. (A) Male paratype (CVULA 7440, SVL = 34.3mm), illustrating the relatively plain dorsal patterning with irregular brown markings and the red iris. (B) Female paratype (CVULA 7434, SVL $=41.6 \mathrm{~mm}$ ), illustrating the 'extravagant' orange-brown patterned phenotype. 

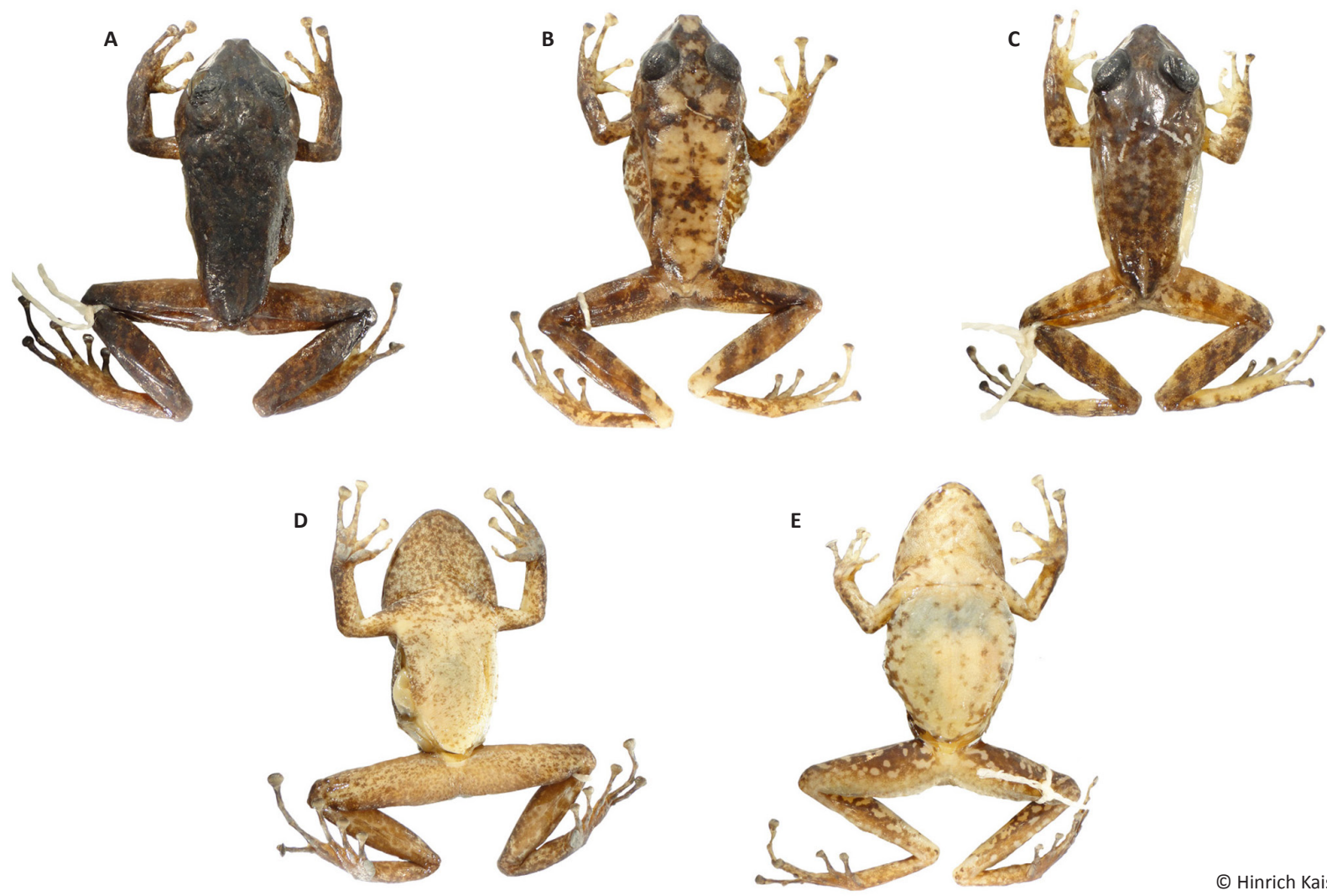

(c) Hinrich Kaiser

Image 9. Dorsal and ventral pattern variation of Pristimantis hoogmoedi sp. nov., illustrating the plain phenotype (A; CVULA 7433, holotype, female, $S V L=51.0 \mathrm{~mm}$ ), the 'extravagant' phenotype (B; CVULA 7434, female, $S V L=41.6 \mathrm{~mm}$ ), and a specimen with an intermediate phenotype (C; CVULA 7437, male, SVL $=32.2 \mathrm{~mm}$ ). The ventral coloration is relatively plain, with a varying degree of spotting. In some specimens, there is a greater density of brown melanophores on the chin, giving it a spotted look (D; CVULA 7433, holotype). In other specimens, the spotting is less dense and more generally distributed across the venter (E; CVULA 7434).

noticeably oval; bifid palmar tubercle; thenar tubercle elongate, covering the base of $\mathrm{F}^{1}$ laterally up to nearly $1 / 4$ of the finger's length; several supernumerary palmar tubercles; (8) fingers with low lateral fringes; (9) few low tubercles on forearm and elbow; (10) few low heel and knee tubercles; inner tarsal fold absent; (11) inner metatarsal tubercle large, elongate; outer small, oval, protuberant, $1 / 8$ size of inner; (12) size of toes $1<$ $2<3<5<4$ (Image 10B, $\mathrm{B}^{\prime}$ ); toe disks oval, disks of $\mathrm{T}^{3-4}$ twice as wide as digit, of $\mathrm{T}^{1-2}$ and $\mathrm{T}^{5} 1.5$ times width of digit; toes with lateral fringes; webbing basal between $\mathrm{T}^{3-4}$ and $\mathrm{T}^{4-5} ; \mathrm{T}^{5}$ slightly longer than $\mathrm{T}^{3}, \mathrm{~T}^{5}$ does not reach ultimate subarticular tubercle on $\mathrm{T}^{4}$; (13) color in life variable, including pale brown or pale orange background with diverse dark brown irregular markings or with mottling (Image 9B); throat with some dark mottling on light background (Image 9D,E); concealed surfaces of the hind limbs orange in life; labial area mottled brown; supratympanic stripe mottled reddishbrown to brown, boomerang-shaped and bent around tympanum, running across one-third of the tympanum itself (e.g., Image 8B); ill-defined lip and limb bars, three tibial bars usually discernible (Images $8 B, 9 B, C$ ); iris color nonmetallic deep red in life (Image $8 A, B$ ); (14) significant sexual size dimorphism present, with females $25 \%$ larger than males on average; axillary amplexus; (15) normal karyotype with $2 n=26$ chromosomes (Image 11).

\section{Comparisons}

Pristimantis hoogmoedi sp. nov. is unique among coastal Cordillera Pristimantis in the following combination of characters: size moderately large, tympanum small with tympanic annulus; middorsal raphe and dorsolateral folds absent; cranial crests absent; basal webbing present between $\mathrm{T}^{3-4}$ and $\mathrm{T}^{4-5}$; iris nonmetallic deep red.

Pristimantis hoogmoedi (characters in parentheses) is here compared with species from the Venezuelan Coastal Range, inclusive of its extension into Trinidad and Tobago (see Table 2). Comparisons with $P$. nubisilva are provided in the species account for that species. Pristimantis anotis is a similarly-sized species, 


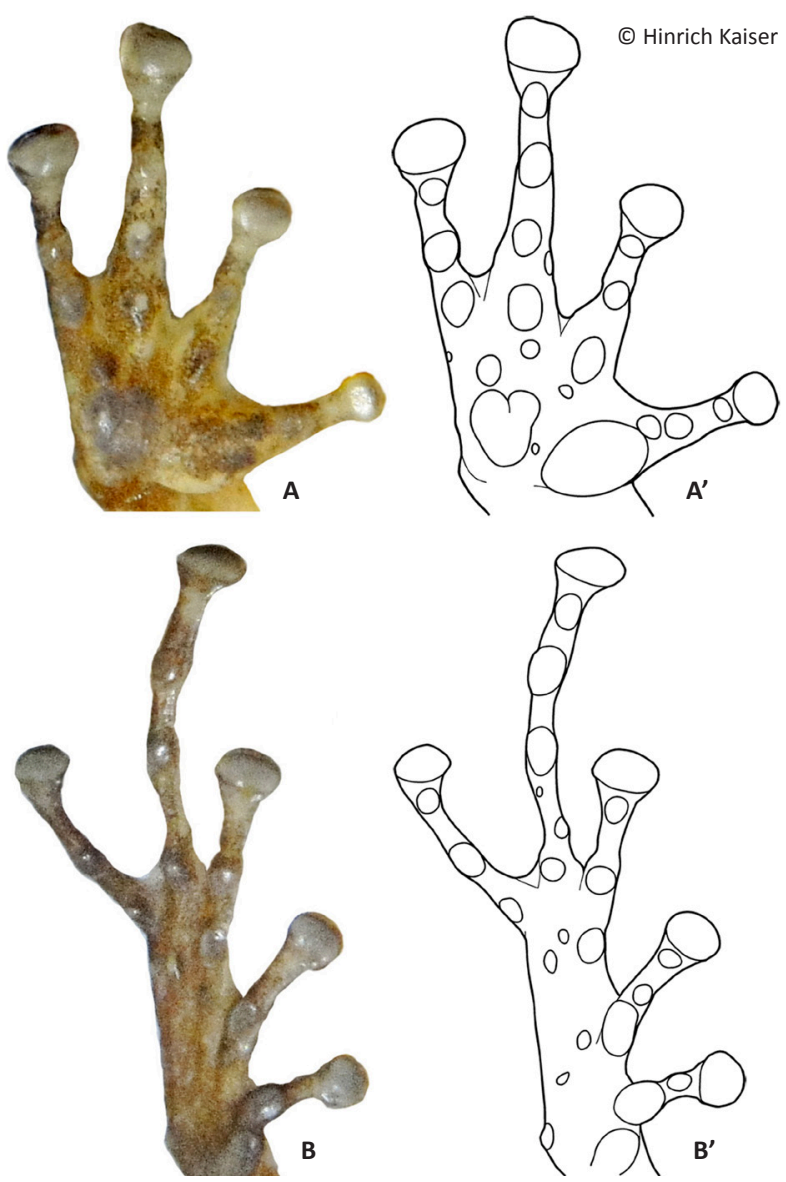

Image 10. Photographs and drawings of hand and foot of Pristimantis hoogmoedi sp. nov. (MHNLS 13346). (A, A') Right hand. (B, B') Right foot.

with females reaching $47 \mathrm{~mm}$ SVL $(51 \mathrm{~mm})$, and it lacks a tympanum (distinct tympanum present). Pristimantis bicumulus is smaller, with females reaching $37 \mathrm{~mm}$ SVL (51 $\mathrm{mm})$, and has an obscured tympanum (distinct), oval dentigerous processes of the vomer (triangular), has a bronze eye in life (red eye), and no webbing between the toes (webbing present between $\mathrm{T}^{3-4}$ and $\mathrm{T}^{4-5}$ ). Pristimantis charlottevillensis, only found on Tobago, is a similarly-sized species with males reaching $31 \mathrm{~mm} \mathrm{SVL}$ $(36 \mathrm{~mm})$, smooth venter (finely areolate), $\mathrm{F}^{1}>\mathrm{F}^{2}\left(\mathrm{~F}^{1}<\mathrm{F}^{2}\right)$, an oval tympanum (round), $\mathrm{T}^{5}<\mathrm{T}^{3}\left(\mathrm{~T}^{5}>\mathrm{T}^{3}\right)$, and vocal slits in males (absent). Pristimantis geminus sp. nov. can be distinguished by its smooth ventral skin (finely areolate), obscured, oval tympanum (distinct, round), ill-defined canthus rostralis (distinct), a tongue without a medial notch (notched), and the presence of a pair of protuberant scapular tubercles (low occipital W-shaped ridge). Pristimantis longicorpus sp. nov. has smooth ventral skin (finely areolate), ill-defined canthus rostralis (distinct), vocal slits in males (absent), and no webbing between the toes (webbing present between $\mathrm{T}^{3-4}$ and $\mathrm{T}^{4-}$ $\left.{ }^{5}\right)$. Pristimantis pariagnomus sp. nov. are much smaller frogs, with the single known male of $13 \mathrm{~mm}$ SVL $(36 \mathrm{~mm})$, smooth ventral skin (finely areolate), obscured, oval tympanum (distinct, round), ill-defined canthus rostralis (distinct), and $\mathrm{F}^{2}>\mathrm{F}^{4}\left(\mathrm{~F}^{2}<\mathrm{F}^{4}\right)$. Pristimantis reticulatus is a smaller species, with males up to $25 \mathrm{~mm}$ in SVL (36mm), with no medial notch in the tongue (notched), and no webbing between the toes (webbing present between $\mathrm{T}^{3-4}$ and $\left.\mathrm{T}^{4-5}\right)$. Pristimantis riveroi is also a smaller species, with males reaching an SVL of $23 \mathrm{~mm}(36 \mathrm{~mm})$, coarsely areolate ventral skin (finely areolate), an obscured, oval tympanum (distinct, round), no medial notch in the tongue (notched), and vocal slits present in males (absent). Pristimantis rozei is much smaller in size, with the only known female of $20 \mathrm{~mm}$ SVL (up to $51 \mathrm{~mm})$, rounded dentigerous processes of the vomers (triangular), $\mathrm{T}^{3}>\mathrm{T}^{5}\left(\mathrm{~T}^{3}<\mathrm{T}^{5}\right)$, and no webbing between the toes (webbing present between $\mathrm{T}^{3-4}$ and $\mathrm{T}^{4-5}$ ). Pristimantis stenodiscus is a much smaller species, with males up to $17 \mathrm{~mm}$ in SVL $(36 \mathrm{~mm})$, with discs pointed at tips (rounded), an obscured tympanum (distinct), and no webbing between the toes (webbing present between $\mathrm{T}^{3-4}$ and $\left.\mathrm{T}^{4-5}\right)$. Pristimantis terraebolivaris is a similarlysized species, with males reaching $36 \mathrm{~mm}$ in SVL $(36 \mathrm{~mm})$, smooth ventral skin (finely areolate), tympanum size $2 / 3$ of the eye diameter ( $1 / 4$ of eye diameter), vocal slits present in males (absent), $F^{1}=F^{2}\left(F^{1}<F^{2}\right), T^{3}>T^{5}\left(T^{3}<\right.$ $\mathrm{T}^{5}$ ), and no webbing between the toes (webbing present between $\mathrm{T}^{3-4}$ and $\mathrm{T}^{4-5}$ ). Pristimantis turpinorum, a Tobago endemic, is a much smaller species, with males reaching $19 \mathrm{~mm}$ in SVL $(36 \mathrm{~mm})$, smooth dorsal skin (finely shagreen), obscured tympanum (distinct), and vocal slits present in males (absent). Pristimantis turumiquirensis, found only in the area of Turumiquire massif, is a similarly-sized species, with females reaching $46 \mathrm{~mm}$ in SVL $(51 \mathrm{~mm})$, smooth ventral skin (finely areolate), $\mathrm{T}^{3}>$ $\mathrm{T}^{5}\left(\mathrm{~T}^{3}<\mathrm{T}^{5}\right)$, and no webbing between the toes (webbing present between $\mathrm{T}^{3-4}$ and $\mathrm{T}^{4-5}$ ). Lastly, $P$. urichi from Trinidad and Tobago is a smaller species, with males reaching $23 \mathrm{~mm}$ in SVL $(36 \mathrm{~mm})$, smooth ventral skin (finely areolate), obscured tympanum (distinct), vocal slits absent in males (present), the presence of a pair of scapular tubercles (absent), no webbing between the toes (webbing present between $\mathrm{T}^{3-4}$ and $\mathrm{T}^{4-5}$ ), and a blue upper iris in life (red eye).

\section{Description of the holotype}

An adult female, SVL $=51.0 \mathrm{~mm}$ (Image 9A,E). Body slender; head wider than body, head $21 \%$ wider than long, $\mathrm{HL} 36 \%$ of SVL, HW $43 \%$ of SVL. Snout subacuminate 


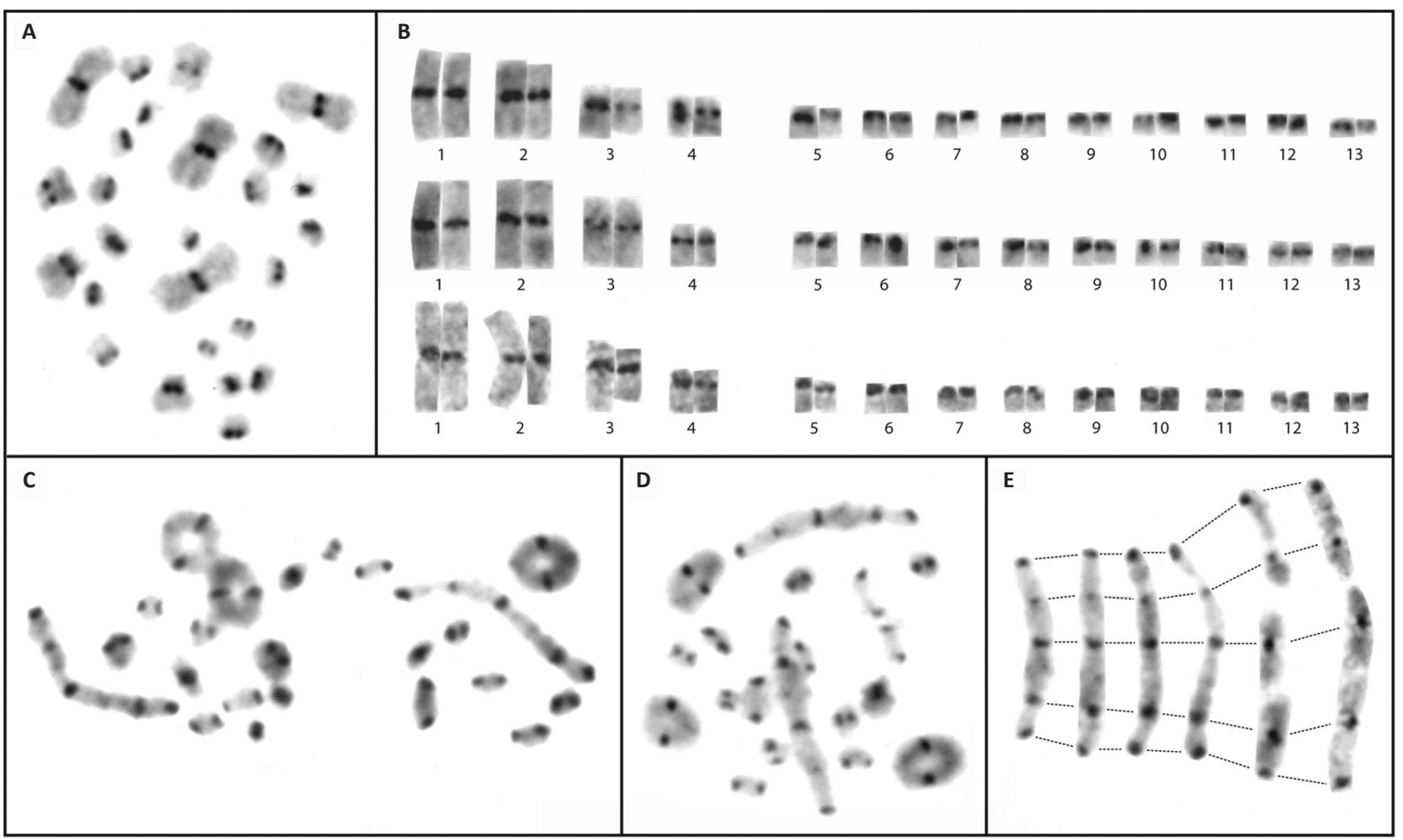

Image 11. (A) C-banded mitotic metaphase and (B) three karyotypes of Pristimantis hoogmoedi sp. nov., showing the location of constitutive heterochromatin in the chromosomes. (C, D) C-banded male meiotic chromosomes in the stage of diakinesis. Note the presence of several large chromosome chains in addition to three large and a number of small ring bivalents. (E) Selected meiotic chains from three different diakineses showing a specific pairing arrangement of the chromosomes along the chains. The corresponding heterochromatic regions are connected by dotted lines.

in dorsal view, subacuminate in profile; $\mathrm{E}-\mathrm{N} 89 \%$ of EYE; nostrils non-protuberant, directed dorsolaterally; canthus rostralis distinct, straight, rounded; loreal region weakly concave. Upper eyelid with a prominent supraocular tubercle. Cranial crests absent. Tympanum small, distinct, $23 \%$ of EYE, surrounded by a tympanic annulus, supratympanic fold present. Choanae small, ovoid, not concealed by palatal shelf of maxillary arch; vomerine dentigerous processes small, protuberant, triangular, with the shortest side of the triangle at the posterior margin of the processes and the apex of the triangle pointing anteriorly, with a single row of teeth along the posterior margin, bearing six teeth on the left and seven teeth on the right process, posterior and medial to the choanae. Tongue round, slightly notched posteriorly, posterior one third free.

Dorsal skin shagreen; middorsal raphe absent; dorsolateral folds absent. Throat and chest smooth, belly and ventral surfaces of thighs slightly granular; posterior surfaces smooth. With few low ulnar, tarsal, and heel tubercles.

Hand medium-sized, $30 \%$ of SVL. Relative lengths of adpressed fingers $1<2<4<3 ; \mathrm{F}^{1}$ slightly shorter than $\mathrm{F}^{2}$, reaching disk on $\mathrm{F}^{2}$ when adpressed. Finger disks much broader than long, disk on $\mathrm{F}^{3}$ over twice the width of adjacent phalanx; all discs horizontally oval except on $\mathrm{F}^{1}$, which is rounded and not distinctly expanded. Disk of $F^{1}$ 1.5 times as wide as adjacent phalanx. Fingers bearing low lateral fringes; webbing absent. Palmar tubercle large, bifid; thenar tubercle large, elongate, ovoid, both low; subarticular tubercles only slightly protuberant, single, oval; several supernumerary tubercles present, large, only slightly protuberant.

Hind limbs long; TIB 54.5\% of SVL (Table 4). Heel reaches the tip of snout when adpressed longitudinally to body axis. Relative lengths of adpressed toes $1<2<3$ $<5<4$. Disk on $\mathrm{T}^{4}$ slightly smaller than disk on $\mathrm{F}^{3}$. Toes with evident lateral fringes, basally webbed between $\mathrm{T}^{3-4}$ and $\mathrm{T}^{4-5}$. Disks distinctly wider than long, horizontally oval. Inner metatarsal tubercle elongate, large, distinct; outer small, protuberant, tubercle-like, ca. $1 / 8$ times size of inner; subarticular tubercles protuberant, single, oval; several small supernumerary tubercles distinguishable under left foot (Image 10B, $\left.B^{\prime}\right)$. 


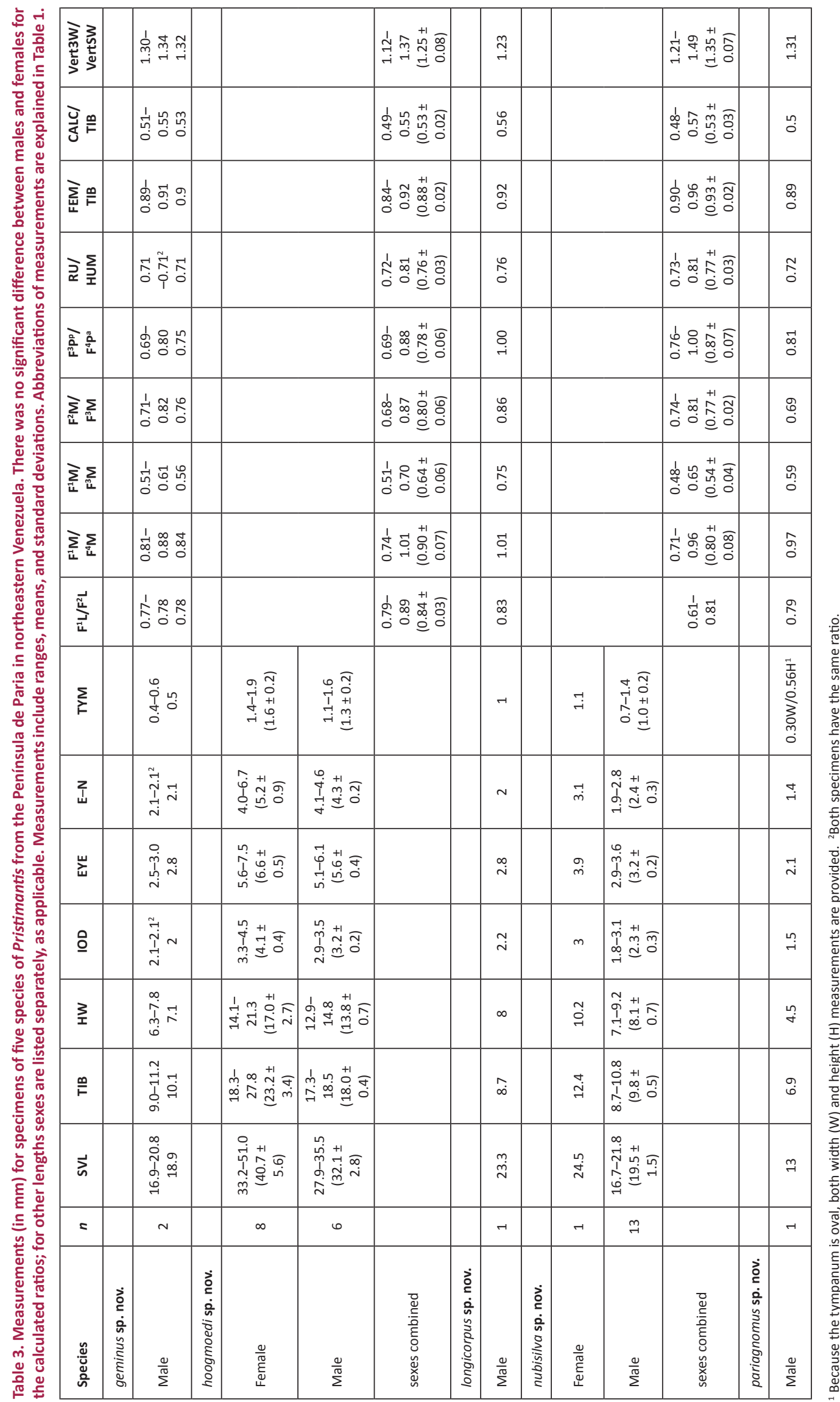




\section{Measurements of holotype (in $\mathrm{mm}$ )}

SVL: 51.0; TIB: 27.8; HL: 18.4; HW: 22.0; IND: 3.5; E-N: 6.7; EYE: 7.5; TYM: 1.7; F²L: 8.6; F²L: 8.8.

\section{Color in Life}

Dorsum and head dark brown, with some faint patterning due to shifts of brown tones noticeable; a dark interorbital chevron present. Flanks lighter than the dorsal area, becoming orange-brown posteroventrally. Limbs with irregular, very dark brown bars; all limb surfaces with some degree of brown-on-brown mottling. Palms of hands and feet brown. Hidden areas of thighs and groin dark orange with some darker mottling. Venter cream, with a profusion of dark spots, extensive on throat and limbs, but diminished on the belly. Iris color deep, non-metallic red.

\section{Color in preservative}

Dorsal color very dark brown with darker markings (Image 9A). The ventral background color is cream (Image 9D,E), the throat covered profusely with dark brown spots, the belly with a lesser concentration of these markings than the throat. The belly has few melanophores and small dark brown spots. The hidden parts of arms and limbs are also profusely covered by a suffusion of dark brown small spots and melanophores; palms and soles are pale gray, disk pads dirty white (Image 10A,B).

\section{Variation}

Males and females lack clear diagnostic characteristics to tell them apart, other than adult size. Sexual size dimorphism is well developed, with females on average $25 \%$ larger than males (Table 3 ). The most reliable character we have observed is a swollen thumb in males, possibly a secondary sexual character; otherwise, we were unable to detect the presence of nuptial pads. All females in the type series agree with the holotype in all basic diagnostic characters. The coloration is not sexually dichromatic.

Pristimantis hoogmoedi has a highly variable coloration, ranging from relatively plain (Image 9A) to 'extravagantly' patterned (Images 8B, 9B). In general, the dorsum is dark brown, usually with small, scattered irregular pale brown reticulations or other brown markings, forming no discernable pattern, but two pale dorsolateral stripes may be present. Flanks are paler than the dorsum, becoming pale yellow ventrally (Image $9 \mathrm{D}, \mathrm{E})$. The throat has some yellowish or darker mottling that sometimes extends onto the belly. The upper lip is dark brown and has lighter brown transverse stripes (e.g., Image 8B), with one distinctive stripe below the eye (e.g., Image 8A). Arms are pale to dark brown with small irregular darker marks, forearms are darker brown than upper arms. Thighs and shanks are also brown, crossed by three transverse dark brown bars. The groin and both the anterior and posterior hidden surfaces of thighs are orange in most specimens, mottled with black and white in others. The iris is deep red, finely reticulated in black.

The coloration of the holotype is also in general agreement with the entire type series. Two specimens (CVULA 7434, 7437) have a peculiar coloration (Image $9 \mathrm{~B})$, comprising an orange background with irregular markings on the head (eyelids, interorbital space dark brown) and a few on the dorsum. In these specimens the flanks are pale brown, with ill-defined diagonal bars and inferiorly white with black reticulation (absent in CVULA 7437). The heel area is pale orange, contrasting with the rest of the tibial and tarsal patterns, which are brown. An uncollected subadult specimen had two yellowish-dorsolateral stripes on brown background. Ventrally, all specimens are similar to the holotype.

\section{Chromosomes}

Pristimantis hoogmoedi is listed as "sp. n. K." by Schmid et al. (2010). All three males and the sole female specimen of $P$. hoogmoedi analyzed show a karyotype with a diploid chromosome number of $2 n=26$ and a fundamental number of $F N=34$. It is composed of four meta- to submetacentric and nine telocentric chromosome pairs (Image 11A,B). C-banding demonstrates that the constitutive heterochromatin is confined to the centromeric regions of all chromosomes. In diakineses of male meiosis, large chromosome chains can be observed in addition to three large and a number of small ring bivalents (Image 11C,D). The analysis of the long chromosome chains reveals that each of them seems to consist of six to seven chromosomes that are terminally joined in a specific order (Image $11 E)$. Such a non-random sequence is explainable by terminal reciprocal translocations between different chromosomes. All three sampled male individuals show the same intriguing pairing arrangement. Such an unusual meiotic pairing arrangement has not been found among 260 other terraranan species, representing $28 \%$ of the 924 species of Terrarana described to date (Schmid et al. 2010). If further studies on more male individuals of $P$. hoogmoedi reveal that this condition has been fixed in the population, it could be concluded that the meiotic segregation of the chromosomes involved in the chain follows an alternate pattern yielding chromosomally 
balanced gametes. Another interesting point would be the analysis of the pairing configurations of lampbrush chromosomes in female meiosis.

\section{Natural history}

Pristimantis hoogmoedi is a nocturnal, essentially rupicolous species, found only in and along rocky creeks with large boulders and waterfalls. We found specimens completely exposed on the rock surface, but always in places difficult to access for humans, such as high walls, crevices, or close to the spray zone of waterfalls. Calling males were seen on small, smooth-leaved plants on the stream's edge, in addition to rocks in the stream. While we were unable to record the advertisement call, both males and females issued release calls (in the form of a 'squeak') when captured. Several egg masses, each containing ca. 15-20 white eggs, were visible inside overhanging dense root tangles, positioned at least $10 \mathrm{~cm}$ deep inside the roots and guarded by males (based on their size) sitting on the roots next to the eggs. Pristimantis hoogmoedi occurs sympatrically with $P$. nubisilva along the edge of streams.

\section{Distribution}

Only known from the southern slope of Cerro Humo, Península de Paria, possibly extended though the cloud forests from 650-1200 m.

\section{Etymology}

The species name is a patronym honoring $\mathrm{Dr}$. Marinus Hoogmoed, prominent Dutch herpetologist and former curator at the RMNH (now Naturalis), whose far-ranging contributions to herpetology have included a special dedication to South American taxa and excellent service to the World Congress of Herpetology. Marinus recognized that some of the Pristimantis specimens collected by Stefan Gorzula in the Península de Paria and deposited at the RMNH might be distinct and new species, but graciously permitted that we describe them.

\section{Pristimantis geminus sp. nov.}

(Images 12A-C, 13A-D, 14A-B, 14A'-B', 20C, 21C)

urn:Isid:zoobank.org:act:922B3E2C-D096-48A3-99B0-231117602967

English name: Paria Twin Landfrog

Spanish name: Ranita Gemela de Paria

\section{Holotype}

USNM [CMD 341] (Images 12A, 13A,C), 7.ix.2001, an adult male from the southern slopes of Cerro Humo $\left(10.7073^{\circ} \mathrm{N} \& 62.6284^{\circ} \mathrm{W}\right)$, elevation $750 \mathrm{~m}$, Estado Sucre, Venezuela, coll. by C. Barrio-Amorós and H. Kaiser.

\section{Paratype}

CVULA 7443 (Image 13B,D), an adult male. It has the same locality data as the holotype and is therefore considered a paratopotype.

\section{Referred specimen}

MBLUZ 381 (adult male) and 382 (adult female) from Cerro Patao, Península de Paria, Estado Sucre, elevation 600m; RMNH 28433, an adult male from Cerro Humo, Península de Paria, Estado Sucre, elevation $750 \mathrm{~m}$.

\section{Diagnosis}

Pristimantis geminus sp. nov. is a small (SVL of the two males we were able to measure: 16.9 and 20.8 $\mathrm{mm}$; SVL of females estimated at 21-25 mm, based on measurements from the photograph in Image 12C; Table 3) member of the $P$. unistrigatus species group (sensu Hedges et al. 2008) characterized by: (1) dorsal skin finely shagreen with middorsal raphe, narrow and low dorsolateral folds (affected by preservation), and two small conical scapular tubercles (Image 12A); ventral skin smooth to finely areolate on throat, chest, and undersides of hind limbs, finely areolate on belly and in groin; cranial crests absent; (2) tympanum slightly oval, very small (TYM $=1 / 6$ of EYE), with tympanic annulus and supratympanic fold (Image 12A,B); E-T about the same as TYM; (3) snout rounded in both dorsal view and profile; canthus rostralis weakly concave in dorsal view, rounded in cross section; E-N $70-75 \%$ of EYE; postrictal tubercles not visible; loreal region concave; (4) IOD nearly equal to EYE; supraocular tubercles present, one raised subconical tubercle on edge of eyelid at mid-eye or towards the posterior part of the eyelid; cranial crests absent; (5) choanae ovoid; dentigerous processes of the vomers small, triangular, with 5-6 teeth on their posterior edge; 12-13 premaxillary teeth; 65-69 maxillary teeth; tongue large, quadrangular, not notched, $1 / 4$ free 

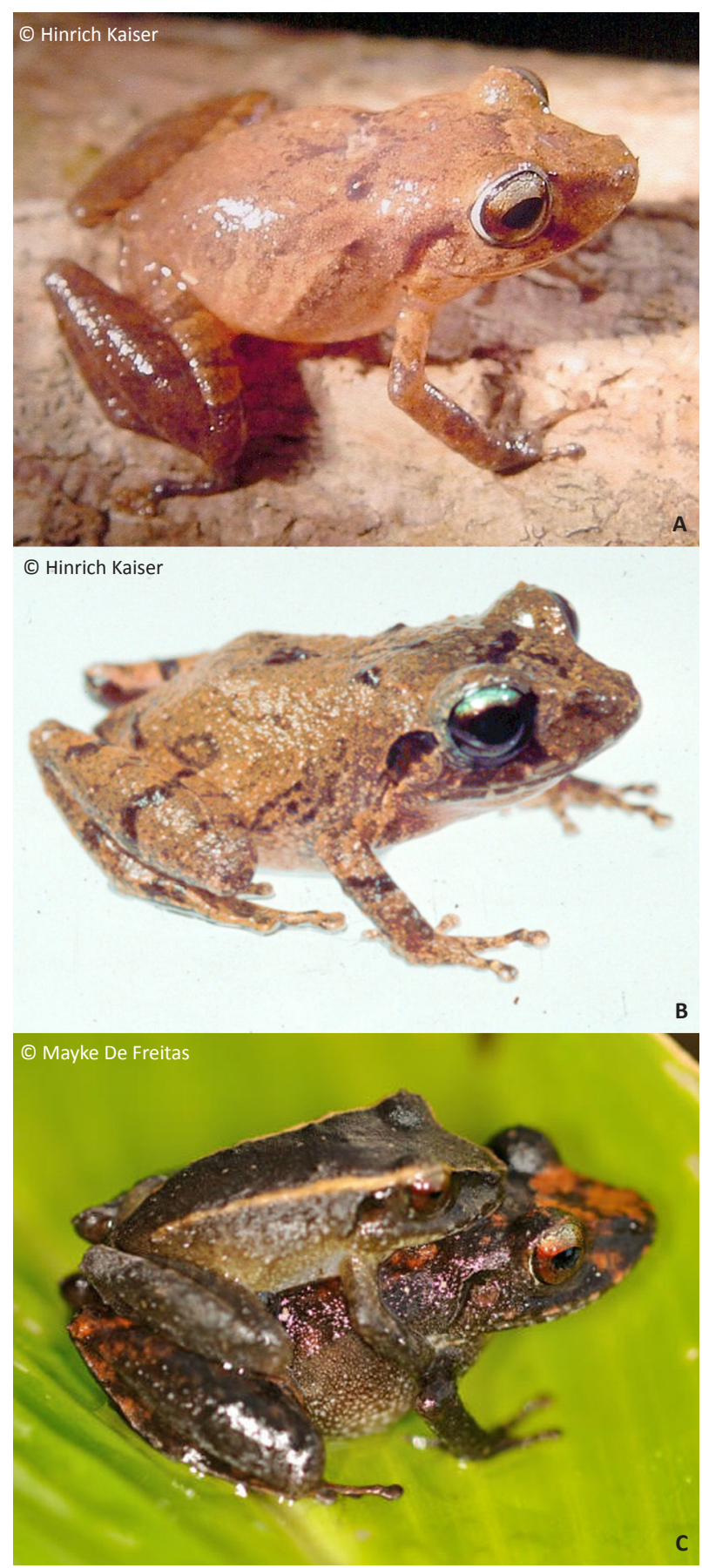

Image 12. Pristimantis geminus sp. nov. from the Península de Paria in northeastern Venezuela in life. (A) Male holotype (USNM [CMD 341], SVL $=20.8 \mathrm{~mm}$ ), illustrating paired scapular spots and associated tubercles. (B) Pristimantis urichi from Trinidad (NMC 35065-1, male, $\mathrm{SVL}=21.2 \mathrm{~mm}$ ), shown to illustrate the similarity with $P$. geminus. Note that the Trinidadian species also possesses dark scapular spots and a more pronounced mid-dorsal chevron, but has a variety of pattern and color differences, most notable a blue upper portion of the iris. (C) Male and female $P$. geminus from Cerro Patao in axillary amplexus (MBLUZ 381-82). The male illustrates the dark brown striped phenotype, showing scapular tubercles and the conical tubercle on the upper eyelid. In these individuals, the coloration is particularly vibrant, perhaps owing to the reproductive activity. posteriorly; (6) vocal slits and nuptial pads absent; (7) size of fingers $1<2<4<3, F^{3}$ about 1.5 times longer than $\mathrm{F}^{1}$; disks on $\mathrm{F}^{2-4}$ expanded, about 1.5 times width of digit, disk on $\mathrm{F}^{1}$ only slightly expanded; subarticular tubercles round and raised; one cordiform palmar tubercle; thenar tubercle oval, extending less than $1 / 4$ length of the thumb laterally; several supernumerary palmar tubercles; (8) fingers with weak lateral fringes; (9) few low tubercles on forearm and elbow; postorbital tubercles very small and only weakly raised; (10) tarsal tubercles absent, subconical tubercles on the heel; inner tarsal fold present; (11) two metatarsal tubercles, inner large and elongate, outer about $1 \%$ size of inner and conical; several supernumerary plantar tubercles present; (12) size of toes $1<2<3<5<4$; toes lacking lateral fringes; basal webbing present between $\mathrm{T}^{4}$ and $\mathrm{T}^{5}$; toes with round disks, only slightly wider than phalanges, only disk on $\mathrm{T}^{4}$ weakly expanded; $\mathrm{T}^{5}$ distinctly longer than $\mathrm{T}^{3}$, reaching past the antepenultimate subarticular tubercle on $\mathrm{T}^{4}$; (13) color in life and preservative similar, dark brown dorsally, sometimes with a brown interorbital bar (Image 12A) but sometimes with light brown stripes along the dorsolateral folds and onto the canthus rostralis (Image 12C); curved supratympanic stripe covering the dorsal portion of the tympanum; stripes on the flanks, and hind limbs, cross-barred with dark brown. Hidden surfaces of the thigh and groin lighter brown with orange spotting. Ventrally throat and chest pale brown with minute white spots, belly white irregularly but profusely spotted with pale brown (Image 13C,D); a pair of dark scapular spots may be present in individuals with a lighter brown dorsal coloration; upper iris color is gunmetal gray, the lower iris reddish bronze; (14) sexual size dimorphism present, with females reaching a $25 \%$ greater size than males (from measurements of amplexed pair; Image 12C); axillary amplexus.

\section{Comparisons}

Pristimantis geminus sp. nov. is unique among Coastal Cordillera Pristimantis in the following combination of characters: size very small (SVL of largest specimen $20.8 \mathrm{~mm}$ ), oval tympanum with tympanic annulus; middorsal raphe, weak dorsolateral folds, a pair of scapular tubercles; cranial crests absent; discs on fingers and toes small, round, scarcely expanded; flanks with diagonal stripes.

Pristimantis geminus (characters in parentheses) is here compared with species from the Venezuelan coastal range, inclusive of its extension into Trinidad and Tobago (see Table 2). Comparisons with P. nubisilva and $P$. hoogmoedi are provided in the species accounts for 

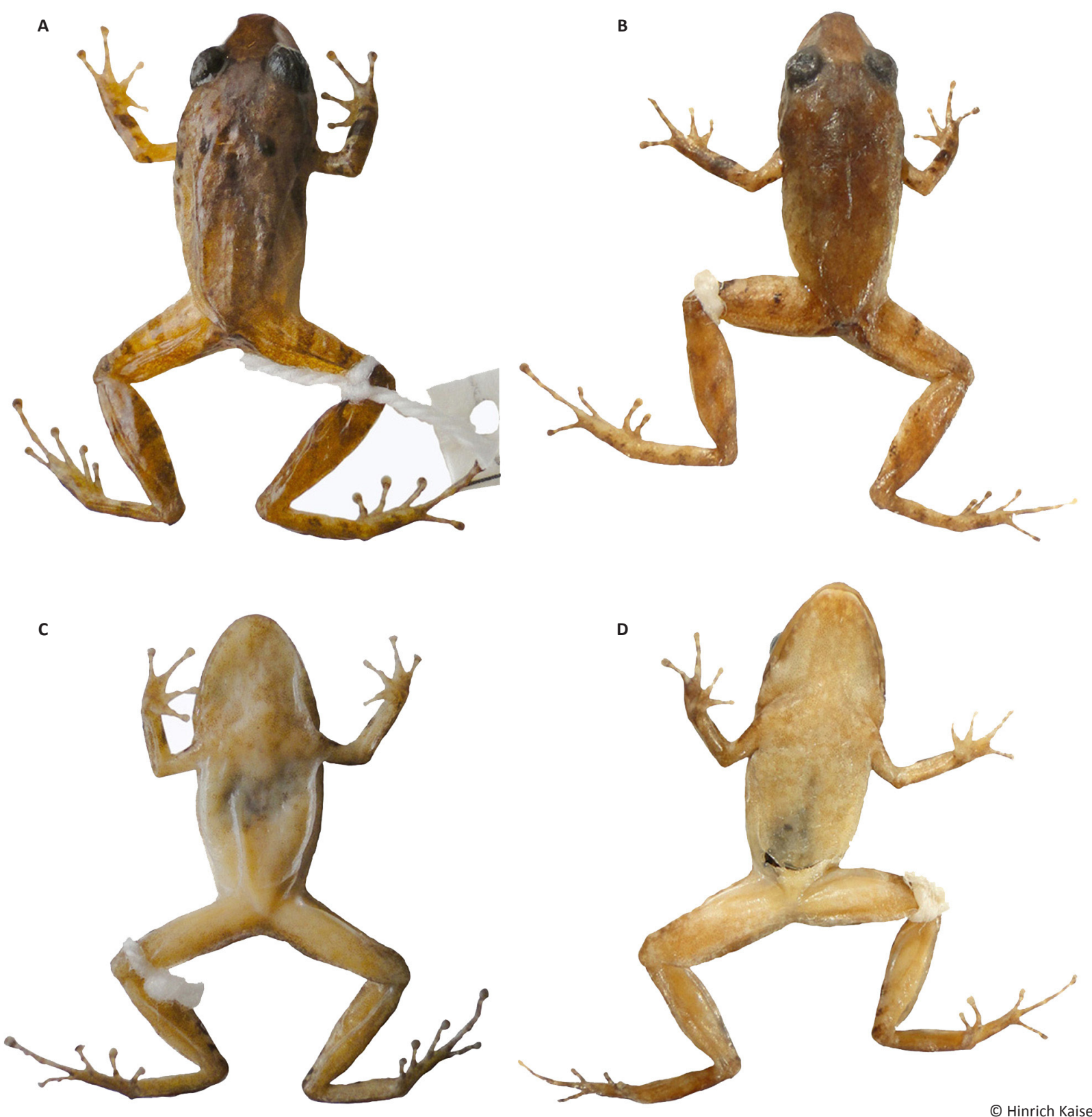

Image 13. Dorsal and ventral pattern variation of Pristimantis geminus sp. nov., illustrating the overall relatively plain coloration. It is noteworthy that the scapular spots are still easily visible in the holotype (A; USNM [CMD 341], male, SVL $=20.8 \mathrm{~mm}$ ) but have faded considerably in the paratopoptype (B; CVULA 7443, male, SVL $=16.9 \mathrm{~mm})$. In contrast, the middorsal raphe is more readily apparent in the paratopotype $(B)$ than in the holotype (A). The ventral coloration is relatively plain, with the profusion of melanophores darkening the appearance of the otherwise cream venter (C, D).

those species. Pristimantis anotis is likely a much larger species (based on the fact that sex ratios in Pristimantis rarely exceed the 2:1 female: male size ratio), with females reaching $47 \mathrm{~mm}$ SVL (males reach $21 \mathrm{~mm}$, females estimated at $21-25 \mathrm{~mm}$ ), has areolate ventral skin (smooth), and it lacks a tympanum (indistinct tympanum present). Pristimantis bicumulus is similarlysized, with males reaching $23 \mathrm{~mm}$ SVL $(21 \mathrm{~mm})$, and has a round tympanum (oval), oval dentigerous processes of the vomer (triangular), and no webbing between the toes (webbing present between $\mathrm{T}^{4}$ and $\mathrm{T}^{5}$ ). The Tobago endemic $P$. charlottevillensis is a larger species with males reaching $31 \mathrm{~mm}$ SVL $(21 \mathrm{~mm})$, distinct tympanum (indistinct), $\mathrm{F}^{1}>\mathrm{F}^{2}\left(\mathrm{~F}^{1}<\mathrm{F}^{2}\right), \mathrm{T}^{5}<\mathrm{T}^{3}\left(\mathrm{~T}^{5}>\mathrm{T}^{3}\right)$, and with vocal slits in males (absent). Pristimantis longicorpus sp. nov. has a distinct, round tympanum (obscured, 
oval), vocal slits in males (absent), and no webbing between the toes (webbing present between $\mathrm{T}^{4}$ and $\left.\mathrm{T}^{5}\right)$. Pristimantis pariagnomus sp. nov. is much smaller, with the single known male of $13 \mathrm{~mm}$ SVL $(21 \mathrm{~mm})$, a medial notch in the tongue (not notched), $F^{2}>F^{4}\left(F^{4}>\right.$ $\left.F^{2}\right), F^{3}=3.5 F^{1}\left(F^{3}=1.5 F^{1}\right)$, and pronounced differences in the shape and size of metatarsal tubercles (Table 2). Pristimantis reticulatus is a similarly-sized species, with males up to $25 \mathrm{~mm}$ in SVL $(21 \mathrm{~mm})$, possessing a distinct, round tympanum (obscured, oval), distinct canthus rostralis (weakly concave and rounded), a truncate snout in dorsal view (round), and no webbing between the toes (webbing present between $\mathrm{T}^{4}$ and $\left.\mathrm{T}^{5}\right)$. Pristimantis riveroi is also a similarly-sized species, with males reaching an SVL of $23 \mathrm{~mm}(21 \mathrm{~mm})$, coarsely areolate ventral skin (finely areolate), distinct canthus rostralis (ill-defined), and vocal slits present in males (absent). Pristimantis rozei is also a small frog, with the only known female of $20 \mathrm{~mm}$ SVL (males up to $21 \mathrm{~mm}$, females estimated at 21-25 mm), areolate ventral skin (smooth), distinct canthus rostralis (weakly concave and rounded), rounded dentigerous processes of the vomers (triangular), $\mathrm{T}^{3}>\mathrm{T}^{5}\left(\mathrm{~T}^{3}<\mathrm{T}^{5}\right)$, and no webbing between the toes (webbing present between $\mathrm{T}^{4}$ and $\mathrm{T}^{5}$ ). Pristimantis stenodiscus is similar in size, with males up to $17 \mathrm{~mm}$ in SVL $(21 \mathrm{~mm})$, disks pointed at tips (rounded), a round tympanum (oval), and no webbing between the toes (webbing present between $\mathrm{T}^{4}$ and $\mathrm{T}^{5}$ ). Pristimantis terraebolivaris is a larger species, with males reaching $36 \mathrm{~mm}$ in SVL $(21 \mathrm{~mm})$, distinct, round tympanum of size $2 / 3$ eye diameter (obscured, oval, $1 / 6$ eye length), $F^{1}=F^{2}$ $\left(\mathrm{F}^{1}<\mathrm{F}^{2}\right)$, and no webbing between the toes (webbing present between $\mathrm{T}^{4}$ and $\mathrm{T}^{5}$ ). Pristimantis turpinorum, a Tobago endemic, is a similarly-sized species, with males reaching $19 \mathrm{~mm}$ in SVL $(21 \mathrm{~mm})$, smooth dorsal skin (finely shagreen), round tympanum (oval), and vocal slits present in males (absent). Pristimantis turumiquirensis, found only in the area of Turumiquire massif, is likely a substantially larger species (as in the case of $P$. anotis), with females reaching $46 \mathrm{~mm}$ in SVL (males up to $21 \mathrm{~mm}$, females estimated at 21-25 $\mathrm{mm}$ ), round tympanum of $1 / 2$ eye diameter (oval, $1 / 6$ eye length), $\mathrm{T}^{3}>\mathrm{T}^{5}\left(\mathrm{~T}^{3}<\mathrm{T}^{5}\right.$ ), and no webbing between the toes (webbing present between $\mathrm{T}^{4}$ and $\mathrm{T}^{5}$ ). Lastly, P. urichi from Trinidad and Tobago is a similarly-sized species, with males reaching $23 \mathrm{~mm}$ in SVL (21 $\mathrm{mm}$ ), round tympanum (oval), vocal slits present in males (absent), no webbing between the toes (webbing present between $\mathrm{T}^{4}$ and $\mathrm{T}^{5}$ ), and a blue upper iris in life (gunmetal).

\section{Description of the holotype}

An adult male, SVL 20.8mm (Images 12A, 13A,B). Body slender; head wider than body, head as long as it is wide, $\mathrm{HL}$ and $\mathrm{HW} 38.7 \%$ of SVL. Snout rounded in dorsal view, rounded in profile; E-N 70\% of EYE; nostrils quite protuberant dorsolaterally; canthus rostralis weakly concave, rounded; loreal region concave. Upper eyelid with a few small tubercles, one slightly larger, subconical tubercle in the posterior half of the eyelid close to its edge. Cranial crests absent. Tympanum very small, almost indistinct, $1 / 5$ of EYE, surrounded by a tympanic annulus, with a swollen supratympanic area (not a fold) covering a very small portion of its posterodorsal section. Choanae moderately large, ovoid, not concealed by palatal shelf of maxillary arch; vomerine dentigerous processes small, triangular, widely separated, slightly oblique, positioned posteriorly and medially to choanae, bearing five teeth each. Tongue quadrangular, not notched posteriorly, posterior one fourth free. Vocal slits absent.

Dorsal skin smooth; middorsal raphe present (Image 12A), ill-defined; lateral and dorsolateral folds present from behind eye to below anterior portion of the sacral area, low, narrow, and indistinct. Throat and ventral surfaces of thighs and shanks smooth, chest skin smooth, venter finely areolate. Few low ulnar and tarsal tubercles present, inner tarsal fold present, heel bearing one small subconical tubercles.

Hand small, $23 \%$ of SVL. Relative lengths of adpressed fingers $1<2<4<3$; $\mathrm{F}^{1}$ slightly shorter than $\mathrm{F}^{2}$; when adpressed, $\mathrm{F}^{1}$ reaches the disk on $\mathrm{F}^{2}$. Finger disks rounded, disk on $\mathrm{F}^{3} 1.5$ width of adjacent phalanx; disk on thumb round, only slightly expanded. Disk on $F^{2} 1.4$ times wider than adjacent phalanx. Nuptial pads not apparent. Weak lateral fringes present on fingers; webbing absent. Cordiform palmar tubercle and ovoid thenar tubercle flat and indistinct; subarticular tubercles low, single, round to oval; multiple supernumerary tubercles, indistinct, one under each finger (Image 14A, $A^{\prime}$ ).

Hind limbs long; TIB 53.8\% of SVL (Table 4). Heel reaches the area between eye and nostril when adpressed longitudinally to the body axis. Relative lengths of adpressed toes $1<2<3<5<4$. $T^{5}$ distinctly longer than $\mathrm{T}^{3}$, surpassing antepenultimate subarticular tubercle of $\mathrm{T}^{4} ; \mathrm{T}^{3}$ barely reaches the antepenultimate subarticular tubercle of $\mathrm{T}^{4}$. Disk on $\mathrm{T}^{4}$ slightly larger than disk on $\mathrm{F}^{3}$. Toes without lateral fringes, unwebbed except basally between $\mathrm{T}^{4}$ and $\mathrm{T}^{5}$. Disks rounded, slightly wider than phalanges. Inner metatarsal tubercle elongate, outer round, small, $1 / 4$ of inner; subarticular tubercles 


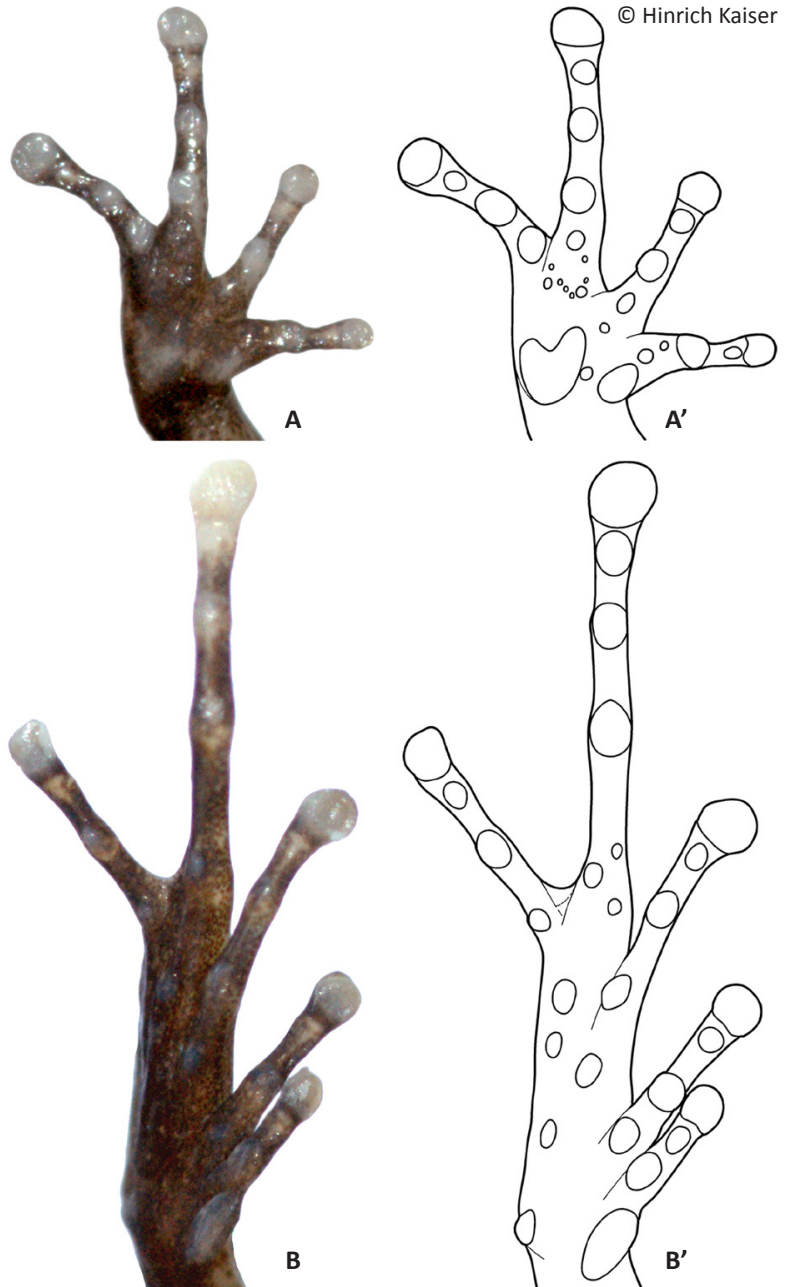

Image 14. Photographs and drawings of hand and foot of the paratopotype of Pristimantis geminus sp. nov. (CVULA 7443). (A, $A^{\prime}$ ) Right hand. (B, B') Right foot.

small, protuberant, single, round to oval (Image 14B, $\left.\mathrm{B}^{\prime}\right)$.

\section{Measurements of holotype (in $\mathrm{mm}$ )}

SVL: 20.8; TIB: 11.2; HL: 8.4; HW: 8.4; IND: 1.8; E-N: 2.1; EYE: 3.0; TYM: 0.6; F ${ }^{1} \mathrm{~L}: 1.8 ; \mathrm{F}^{2} \mathrm{~L}: 2.5$.

\section{Color in life}

Coloration is here described based on the holotype, the paratypes, and the photograph of the amplexed pair (Image 12C). Dorsum dark brown without any distinguishable pattern except for a pair of scapular spots that coincide with a conical tubercle (Image 12A). The scapular spots may be difficult to see in a very dark ground color, but the tubercles are visible (Image 12C). A dark brown chevron is visible at the center of the dorsum, but it is difficult to discern on a dark background color. A paler brown interorbital bar is visible between
Table 4. Ratios of key measurements for species of Pristimantis from the Península de Paria, Trinidad (Tri), and Tobago (Tob). Measurements used are from the holotype of each species. For TIB/SVL we consider species with ratios $\geq 0.50$ as long-legged (***), those with ratios between 0.40 and 0.49 as having moderately long legs $\left({ }^{* *}\right)$, and those with ratios $<0.40$ as short-legged $\left({ }^{*}\right)$. Similarly, we divide E-N/EYE ratios, as indicators of snout length, into three groups: those with long snouts $\left(\geq 1.00 ;{ }^{\circ}\right)$, medium snouts $(0.80-$ $\left.0.99 ;^{\circ \circ}\right)$ and short snouts $\left(<0.80 ;{ }^{\circ}\right)$. Abbreviations of measurements are listed in Table 1.

\begin{tabular}{|l|c|c|c|}
\hline Species & TIB/SVL & HW/SVL & E-N/EYE \\
\hline anotis & $0.54^{* * *}$ & 0.40 & $1.15^{\circ \circ}$ \\
\hline bicumulus & $0.49^{* *}$ & 0.37 & $0.82^{\circ \circ}$ \\
\hline charlottevillensis ${ }^{\text {Tob }}$ & $0.60^{* * *}$ & 0.39 & $0.93^{\circ \circ}$ \\
\hline geminus sp. nov. & $0.54^{* * *}$ & 0.38 & $0.70^{\circ}$ \\
\hline hoogmoedi sp. nov. & $0.55^{* * *}$ & 0.42 & $0.89^{\circ \circ}$ \\
\hline longicorpus sp. nov. & $0.37^{*}$ & 0.34 & $0.71^{\circ}$ \\
\hline nubisilva sp. nov. & $0.51^{* * *}$ & 0.42 & $0.79^{\circ}$ \\
\hline pariagnomus sp. nov. & $0.53^{* * *}$ & 0.35 & $0.67^{\circ}$ \\
\hline reticulatus & $0.52^{* * *}$ & 0.40 & $0.94^{\circ \circ}$ \\
\hline riveroi & $0.48^{* *}$ & 0.38 & $1.00^{\circ \circ}$ \\
\hline rozei & $0.50^{* * *}$ & 0.39 & $0.71^{\circ}$ \\
\hline stenodiscus & $0.45^{* *}$ & 0.39 & $1.15^{\circ \circ 0}$ \\
\hline terraebolivaris & $0.60^{* * *}$ & 0.35 & $0.97^{\circ \circ}$ \\
\hline turpinorum ${ }^{\text {Tob }}$ & $0.54^{* * *}$ & 0.35 & $0.64^{\circ}$ \\
\hline turumiquirensis & $0.53^{* * *}$ & 0.35 & $1.00^{\circ \circ}$ \\
\hline urichi ${ }^{\text {Tri }}$ & $0.51^{* * *}$ & 0.39 & $0.91^{\circ \circ}$ \\
\hline
\end{tabular}

the eyes in the holotype, slightly anterior to the central portion of the eyes, but this area is uniformly dark in some males (Image 12C). The frontonasal area is slightly paler than the rest of the body in the paratype (CVULA 7443), and there is no canthal stripe, whereas the male in the photograph (Image 12C) has a uniformly dark brown dorsum, framed by two distinct light brown dorsolateral lines that extend onto the snout. The patterning on the head of the female in the photograph (Image 12C) is irregular, consisting of patches of orange on a deep brown background. There is a dark, boomerang-shaped supratympanic stripe extending from just behind the eye to above the upper arm (Image 12A,C). The flanks are of the same color as the dorsum, but there is a slight patterning of diagonal alternating paler brown and darker brown striping, and the color becomes gradually lighter in the lower lateral regions. The arms are brown with ill-defined bars or irregular spots. The thighs are brown, with dark brown cross bars that are easily visible in individuals with lighter background coloration (e.g., Image 13A,B); the shanks are darker with ill-defined dark brown cross bars. The upper lips are brown or 
orange and crossed by darker brown perpendicular bars or spots. The upper part of the iris is pale brownish gray (gunmetal) with fine black reticulation, and the lower half has a reddish hue. The hidden portions of the thighs have small, variable brownish-reddish spots on a dark brown background.

\section{Color in preservative}

Dorsum plain, pale brown, with few signs of patterning (Image 13A,B); the middorsal raphe appears slightly paler under magnification (Image 13B). Pale brown interorbital bar ill-defined. Arms from above very pale brown; forearms darker, crossed by one wide and two narrower dark brown bars. Fingers dark brown with narrow lighter brown rings. Thighs pale brown, crossed by dark brown bars; shanks, tarsi, and feet dark brown with darker ill-defined crossbars. The vent area is covered by a triangular dark brown mark that includes the inferior part of the thighs, delimited with a narrow black line (Image 13A,B). Ventrally, throat and chest pale brown with minute white spots, more contrasting on the chin and edges of lower mandible, belly white irregularly but profusely spotted with pale brown (Image 13C,D); undersides of thighs dirty white anteriorly, profusely spotted with dark brown melanophores; shanks and tarsi dark brown; palms and soles dark brown with white subarticular tubercles and disks.

\section{Natural history}

The holotype and paratype were collected while prospecting epiphyte plants during the day. Both specimens were found in bromeliads ca. $4 \mathrm{~m}$ above the ground. An amplexed pair (MBLUZ 381-82) referable to this species was encountered at ground level on a rock in a small creek. Reproductive activity appears to result in a particular vibrant, deep coloration on both male and female (Image 12C), whereas it is relatively pale in the male holotype (Image 12A).

\section{Distribution}

Only known from the southern slopes of Cerro Humo and Cerro Patao, Península de Paria, possibly extended though the cloud forests from 650-1200 m.

\section{Etymology}

The species name is a noun in apposition. Because of the striking similarity of this species with $P$. stenodiscus and $P$. urichi, we consider the morphology of the new species akin to that of twins (geminus, Latin = twin).
Pristimantis longicorpus sp. nov. (Images 15A-B, 16A-B, 16A'-B', 20D, 21D)

urn:Isid:zoobank.org:act:897CB077-6DB4-4793-BEC9-6C5F9F1A3F54

English name: Long-Bodied Paria Landfrog

Spanish name: Ranita Alargada de Paria

\section{Holotype}

CVULA 7444 (Image 15A,B), 7.ix.2001, an adult male from the southern slopes of Cerro Humo $\left(10.7073^{\circ} \mathrm{N} \&\right.$ $62.6284^{\circ} \mathrm{W}$ ), elevation $750 \mathrm{~m}$, Estado Sucre, Venezuela, coll. by C. Barrio-Amorós and H. Kaiser.

\section{Diagnosis}

Pristimantis longicorpus sp. nov. is a small (SVL for single adult male, $22.5 \mathrm{~mm}$; Table 3 ) member of the $P$. unistrigatus species group (sensu Hedges et al. 2008) characterized by: (1) dorsal skin smooth anteriorly, bearing tubercles posteriorly, four large symmetrically arranged tubercles present on each side of the sacrum, middorsal raphe present, dorsolateral folds absent; ventral skin smooth to finely areolate in preservative; cranial crests absent; (2) tympanum round, distinct, about $35 \%$ of EYE, with tympanic annulus and supratympanic fold; E-T ca. 1.5 times TYM; (3) snout truncate in dorsal view, subacuminate in profile; canthus rostralis ill-defined, sinuous in dorsal view, rounded in cross section; three distinctive, cream-colored postrictal tubercles present posteriorly to the commissure of the mouth in the straight-line area between the tympanum and the insertion of the arm, their size about that of the narial opening; (4) IOD about 2/3 EYE; upper eyelid with two flat tubercles; (5) choanae small, rounded; dentigerous processes of the vomers large, triangular, widely separated, with 8-9 teeth on the posterior margin of each process; $10-11$ premaxillary teeth; 72 maxillary teeth; tongue large, oval, notched medially, posterior half free; (6) vocal slits and nuptial pads absent; (7) size of fingers $1<2=4<3, F^{3}$ about 1.5 times as long as $\mathrm{F}^{1} ; \mathrm{F}^{1}$ shorter than $\mathrm{F}^{2}$; finger disks on $\mathrm{F}^{2-4}$ only slightly expanded, lacking on $\mathrm{F}^{1}$ (Image 16A, $\mathrm{A}^{\prime}$ ); subarticular tubercles round and only slightly raised; a single rounded palmar tubercle; thenar tubercle rounded and relatively small, covering only the base of $F^{1}$; very few supernumerary palmar tubercles; (8) fingers without lateral fringes; (9) ulnar tubercles present in a row of indistinct flat and round tubercles on the undersides of the forearms; (10) tarsal tubercles absent, calcars absent; inner tarsal fold absent; (11) two metatarsal tubercles present; inner metatarsal tubercle flat and oval; outer metatarsal tubercle moderately large, oval, 

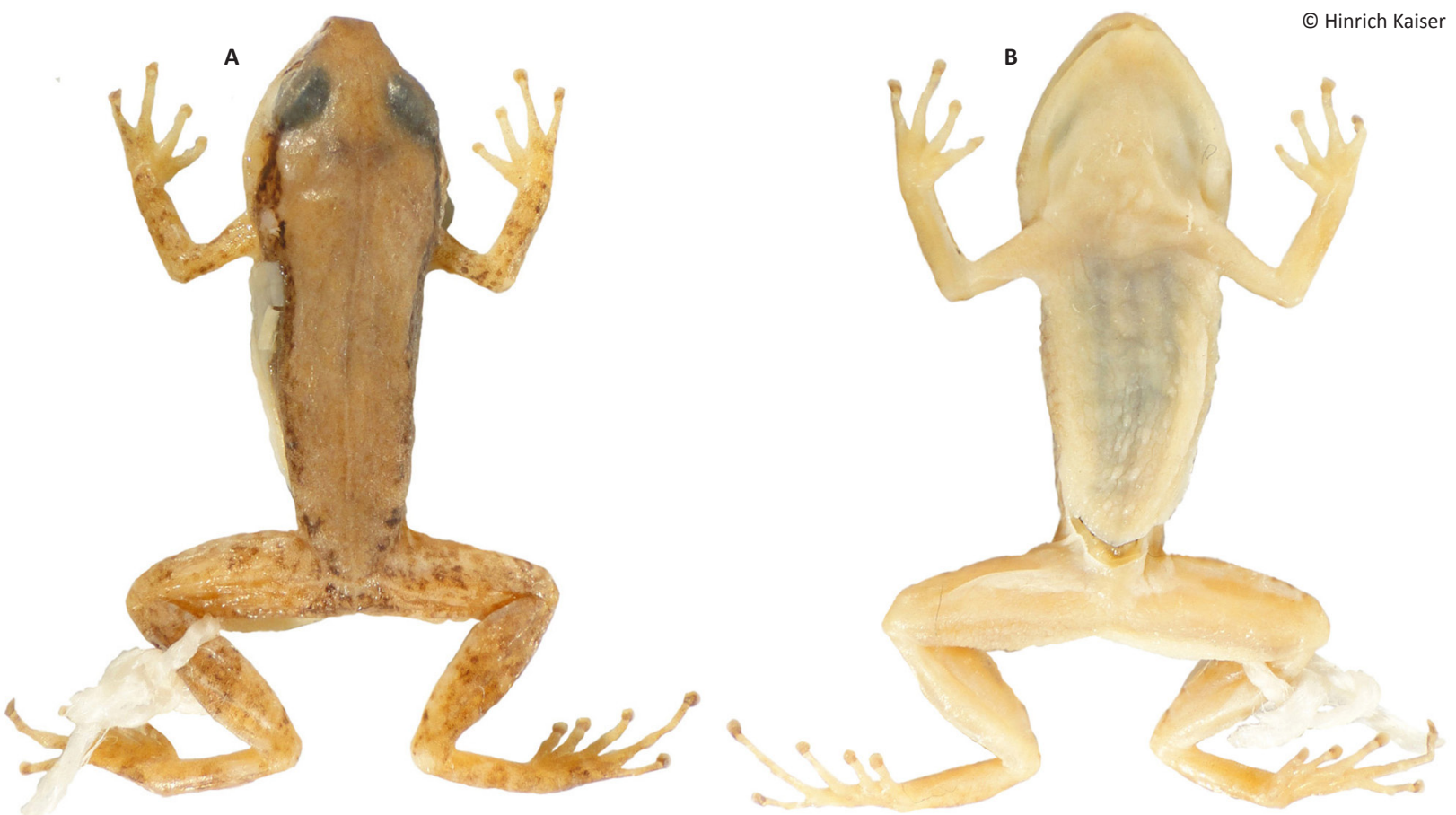

Image 15. Male holotype of Pristimantis longicorpus sp. nov. (CVULA 7444, SVL = 22.5) from the Península de Paria in northeastern Venezuela in (A) dorsal and (B) and ventral views.

protuberant, half the size of inner (Image 16B, $\left.\mathrm{B}^{\prime}\right)$; (12) size of toes $1<2<3<5<4$; toes without lateral fringes, not webbed; toes with only slightly expanded, rounded disks; $\mathrm{T}^{3}$ slightly longer than $\mathrm{T}^{5}$, neither toe reaching the penultimate subarticular tubercle on $\mathrm{T}^{4}$; (13) color pattern dorsoconcolor, with a pale brown uniform dorsum, darker sides, and white belly.

\section{Comparisons}

Pristimantis longicorpus sp. nov. is unique among Coastal Cordillera Pristimantis in the following combination of characters: size small (SVL of only known male specimen $22.5 \mathrm{~mm}$ ), body shape elongate, quadrangular; tympanum large with tympanic annulus, $35 \%$ of EYE; large dentigerous processes; venter immaculate; disc on $\mathrm{F}^{1}$ absent; discs on $\mathrm{F}^{2-4}$ only slightly expanded, rounded, middorsal raphe present, dorsolateral folds absent; cranial crests absent.

Pristimantis longicorpus (characters in parentheses) is here compared with species from the Venezuelan Coastal Range, inclusive of its extension into Trinidad and Tobago (see Table 2). Comparisons with P. nubisilva, $P$. hoogmoedi, and $P$. geminus are provided in the species accounts for those species. Pristimantis anotis is a much larger species, with females reaching $47 \mathrm{~mm}$ SVL (SVL of only known male specimen $23 \mathrm{~mm}$ ), has areolate ventral skin (smooth), and it lacks a tympanum (distinct tympanum present). Pristimantis bicumulus is similarly-sized, with males reaching $23 \mathrm{~mm}$ SVL $(23 \mathrm{~mm})$, has areolate ventral skin (smooth), and has an obscured tympanum (distinct), oval dentigerous processes of the vomer (triangular), distinct canthus rostralis (illdefined), vocal slits absent in males (present), and significantly different shape and size of metatarsal tubercles (Table 2). Pristimantis charlottevillensis, only found on Tobago, is a larger species with males reaching $31 \mathrm{~mm} \mathrm{SVL}(23 \mathrm{~mm})$, oval tympanum (round), $F^{1}>F^{2}\left(F^{1}<\right.$ $\left.F^{2}\right), T^{5}<T^{3}\left(T^{5}>T^{3}\right)$, and with vocal slits in males (absent). Pristimantis pariagnomus sp. nov. are much smaller frogs, with the single known male of $13 \mathrm{~mm}$ SVL $(23 \mathrm{~mm})$, an obscured, oval tympanum (distinct, round), vocal slits present in males (absent), $F^{2}>F^{4}\left(F^{4}>F^{2}\right)$, and $F^{3}=$ 3.5 $\mathrm{F}^{1}\left(\mathrm{~F}^{3}=1.5 \mathrm{~F}^{1}\right)$. Pristimantis reticulatus is a similarlysized species, with males up to $25 \mathrm{~mm}$ in SVL $(23 \mathrm{~mm})$, possessing finely areolate ventral skin (smooth), distinct canthus rostralis (ill-defined), no medial notch in the tongue (notched), and several differences in the shape and size of metatarsal tubercles (Table 2). Pristimantis riveroi is a similarly-sized species, with males reaching an SVL of $23 \mathrm{~mm}(23 \mathrm{~mm})$, coarsely areolate ventral skin (smooth), obscured, oval tympanum (distinct, round), distinct canthus rostralis (ill-defined), and no notch in posterior border of tongue (notched). Pristimantis rozei is a small frog, with the only known female of 
$20 \mathrm{~mm}$ SVL (only known specimen $23 \mathrm{~mm}$ ), areolate ventral skin (smooth), distinct canthus rostralis (illdefined), rounded dentigerous processes of the vomers (triangular), and $\mathrm{T}^{3}>\mathrm{T}^{5}\left(\mathrm{~T}^{3}<\mathrm{T}^{5}\right)$. Pristimantis stenodiscus is slightly smaller in size, with males up to $17 \mathrm{~mm}$ in SVL $(23 \mathrm{~mm})$, disks pointed at tips (rounded), an obscured tympanum (distinct), and several differences in size and shape of metatarsal tubercles (see Table 2). Pristimantis terraebolivaris is a larger species, with males reaching $36 \mathrm{~mm}$ in SVL $(23 \mathrm{~mm})$, canthus rostralis distinct and straight (ill-defined, concave), $F^{1}=F^{2}\left(F^{1}\right.$ $\left.<\mathrm{F}^{2}\right)$, and $\mathrm{T}^{3}>\mathrm{T}^{5}\left(\mathrm{~T}^{3}<\mathrm{T}^{5}\right)$. Pristimantis turpinorum, a Tobago endemic, is a similarly-sized species, with males reaching $19 \mathrm{~mm}$ in SVL $(23 \mathrm{~mm})$, obscured tympanum (distinct), distinct canthus rostralis (ill-defined), and no webbing between the toes (webbing present between $\mathrm{T}^{4}$ and $\left.\mathrm{T}^{5}\right)$. Pristimantis turumiquirensis, found only in the area of Turumiquire massif, is a substantially larger species, with females reaching $46 \mathrm{~mm}$ in SVL (males up to $23 \mathrm{~mm}$ ), a distinct canthus rostralis (ill-defined), two rows of vomerine teeth (a single row), longer legs with TIB 53\% of SVL (37\%), and $\mathrm{T}^{3}>\mathrm{T}^{5}\left(\mathrm{~T}^{3}<\mathrm{T}^{5}\right)$. Lastly, P. urichi from Trinidad and Tobago is a similarly-sized species, with males reaching $23 \mathrm{~mm}$ in SVL $(23 \mathrm{~mm})$, obscured tympanum (distinct), rounded snout (truncate), distinct canthus rostralis (ill-defined), two palmar tubercles (one), considerable differences in the morphology of metatarsal tubercles (Table 2), and a blue upper iris in life (bronze).

\section{Description of the holotype}

An adult male, $\mathrm{SVL}=22.5 \mathrm{~mm}$. Body elongate; head wider than body, $5 \%$ wider than long; $\mathrm{HL} 34 \%$ of SVL; HW $36 \%$ of SVL. Snout truncate in dorsal view, subacuminate in profile, coming to a slight point at its tip; E-N 71\% of EYE; nostrils slightly protuberant, directed dorsolaterally; canthus rostralis poorly defined, sinuous in dorsal view, rounded in profile; loreal region weakly concave. Upper eyelid with two small, flat tubercles, possibly subconical in life. Cranial crests absent. Tympanum distinct, 35\% of EYE, surrounded by a tympanic annulus, with a flat, darkly colored supratympanic fold covering a small portion of its dorsal edge. Choanae small, round, close to the maxillary arch, not concealed by palatal shelf; vomerine dentigerous processes large, triangular, widely separated, bearing 9 (right) and 8 (left) teeth, posterior and medial to choanae. Vocal slits short, in the very posterior part of the mouth. Tongue oval, slightly notched posteriorly, posterior half free. Testes dark gray, one-third of the size of kidneys.

Dorsal skin smooth anteriorly, bearing tubercles
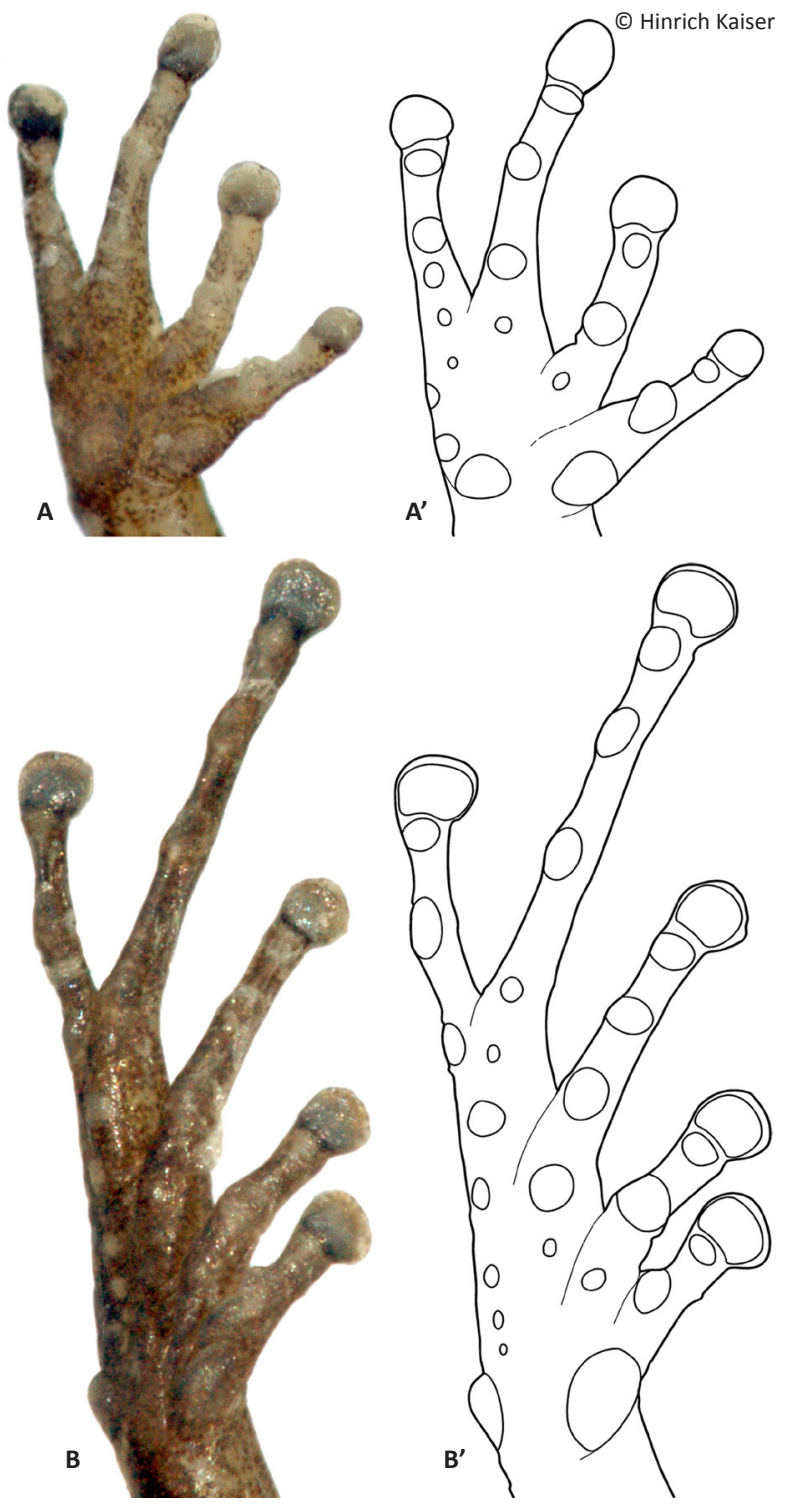

Image 16. Photographs and drawings of hand and foot of the holotype of Pristimantis longicorpus sp. nov. (CVULA 7444). (A, A') Right hand. (B, B') Right foot.

posteriorly, with four large tubercles symmetrical on each side of the sacral part of the body; one large tubercle posteroventral to tympanum present; middorsal raphe present from behind the head to the cloacal opening; status of dorsolateral folds inconclusive due to preservation. Throat and chest finely areolate, belly and ventral surfaces of thighs areolate; flanks areolate inferiorly, posterior surfaces smooth. Few low ulnar, tarsal, and heel tubercles present.

Hand small, $21 \%$ of SVL. Relative lengths of adpressed fingers $1<2<4=3$; when adpressed, $F^{1}$ reaching disk on $\mathrm{F}^{2}$. Finger disks rounded. Disk on $\mathrm{F}^{3} 1.4$ times wider 
than adjacent phalanx, disk on $\mathrm{F}^{2} 1.8$ times wider than adjacent phalanx. Lateral fringes on fingers absent; webbing absent. Nuptial pads absent. Palmar tubercle rounded; thenar tubercle rounded, low, indistinct (Image 16A'); subarticular tubercles single, round, low, with the exception of the subarticular tubercle on $F^{1}$, which is protuberant (Image 16 $\mathrm{A}^{\prime}$ ); few supernumerary tubercles. There is a row of indistinct flat and round ulnar tubercles on the undersides of the forearms.

Hind limbs short; TIB 37\% of SVL (Table 4). Heel reaches the tympanum when adpressed longitudinally to the body axis. Relative lengths of adpressed toes 1 $<2<3<5<4$, neither $\mathrm{T}^{3}$ nor $\mathrm{T}^{5}$ reaching the second subarticular tubercle on $\mathrm{T}^{4}$ (Image $16 \mathrm{~B}^{\prime}$ ). Toes without lateral fringes, unwebbed. Disks rounded, slightly wider than phalanges. Inner metatarsal tubercle oval; outer moderately large, half the size of inner, protuberant, oval (Image 16B,B'); subarticular tubercles distinct, single, round; a few indistinct supernumerary tubercles, especially in a line along the outer margin of the plantar surface (Image 16B').

\section{Measurements of holotype (in $\mathrm{mm}$ )}

SVL: 22.5; TIB: 9.1; HL: 7.6; HW: 8.0; IND: 1.7; E-N: 2.0; EYE: 2.8; TYM: 1.0; F²L: 2.1; F²L: 2.5 .

\section{Color in life}

Unknown. We initially considered this specimen to belong to $P$. nubisilva and recognized only after preservation that it was a distinct form. We therefore are unable to provide information on color in life.

\section{Color in Preservative}

Dorsal area uniformly pale brown, bordered laterally by a dark brown stripe at the transition from dorsal to ventral skin; some of the larger tubercles in the sacral area very dark brown; middorsal raphe slightly paler than the rest of the dorsum; extremities pale brown, arms spotted dark brown; legs spotted and flecked in dark brown (Image 15A). Canthal stripe dark brown, wide, covering nares; supratympanic stripe wide, dark brown, boomerang-shaped, covering upper edge of tympanum and turning behind tympanum towards the upper arm; three postrictal tubercles forming white stripe from behind the tympanum to the upper arm. A narrow longitudinal, white stripe exists symmetrically on the posterior side of each thigh, and there is a well-defined stripe from the heel to the outer metatarsal tubercle on each tarsus. Ventrally (Image 15B), the throat is white with a profusion of melanophores on the chin; the chest is white with fewer melanophores (indistinct without microscope), and the belly is immaculate white. The undersides of the forearms are spotted with a profusion of melanophores; the flat tubercles on the inferior side of the forearms are white; palms are pale brown. The undersides of the thighs are white posteriorly, but receive an invasion of melanophores from the anterior side; shanks are pale brown and tarsi are dark brown; soles are brown.

\section{Natural history}

The only known individual of Pristimantis longicorpus was collected along with a series of $P$. nubisilva and was originally believed to belong to that species. The specimen was a non-calling male in an area where males of $P$. nubisilva had set up calling sites at night. No further data were obtained for the species.

\section{Distribution}

Only known from the southern slope of Cerro Humo in the Península de Paria. Its range is predicted to extend throughout the cloud forests at altitudes from 650-1200 m.

\section{Etymology}

The species name longicorpus is a composite noun, derived from the Latin words "Iongus" = long, and "corpus" = body, in reference to the rather elongate body of this species in comparison to its hind limb size. 
Pristimantis pariagnomus sp. nov.

(Images 17, 18A-B, 19A-B, 19A'-B', 20E, 21E)

urn:Isid:zoobank.org:act:A2F506B3-5C58-4C2F-8084-D4575E326923

English name: Paria Dwarf Landfrog

Spanish name: Ranita Enana de Paria

\section{Holotype}

MHNLS 14456 (Image 18), 15.xi.1978, an adult male from the southern slopes of Cerro Humo $\left(10.7073^{\circ} \mathrm{N} \&\right.$ $\left.62.6284^{\circ} \mathrm{W}\right)$, elevation $780 \mathrm{~m}$, Estado Sucre, Venezuela, coll. by S. Gorzula.

\section{Diagnosis}

Pristimantis pariagnomus sp. nov. appears to be a minute (SVL of the only known specimen, an adult male, $13.0 \mathrm{~mm}$; the individual in Image 17 was not measured and escaped during the attempt to capture it; it was also very small and similar in size to the holotype) member of the $P$. unistrigatus species group (sensu Hedges et al. 2008) characterized by: (1) dorsal skin nearly smooth in preservative, with two parallel rows of small tubercles beginning behind the eyes and extending along the lateral portion of the dorsum, middorsal raphe present, dorsolateral folds absent; ventral skin smooth to finely granular in preservative; cranial crests absent (Image
17); (2) tympanum indistinct, oval (tympanum height nearly twice as long as tympanum width), tympanum width about $14 \%$ of EYE, with nearly indistinct tympanic annulus but with prominent supratympanic fold; E-T nearly twice TYM; (3) snout rounded in dorsal view (Image 18A), truncate in profile; canthus rostralis ill-defined, concave in dorsal view, rounded in cross section; loreal region concave; two cream-colored postrictal tubercles present posteriorly to the commissure of the mouth in the straight-line area between the tympanum and the insertion of the arm, their size about that of the narial opening; (4) IOD about 2/3 EYE; upper eyelid with a single protuberant tubercle in the posterior half; (5) choanae small, ovoid; dentigerous processes of the vomers small, triangular, widely separated, with three teeth on the posterior margin of each process; eight premaxillary teeth; 25 maxillary teeth; tongue large, elongate, notched medially, posterior half free; (6) vocal slits absent, nuptial pads absent; (7) size of fingers $1<4<2=$ $3, F^{3}$ about three 3.5 as long as $F^{1} ; F^{1}$ much shorter than $F^{2}$ $\left(F^{1} \mathrm{~L}=50 \% \mathrm{~F}^{2} \mathrm{~L}\right)$; finger disks on $\mathrm{F}^{2-4}$ expanded, disk absent on $\mathrm{F}^{1}$ (Image 19A, $\mathrm{A}^{\prime}$ ); subarticular tubercles round and only slightly raised; a single cordiform palmar tubercle; thenar tubercle rounded and very large, covering the entire base of $\mathrm{F}^{1}$; several small supernumerary palmar tubercles present; (8) fingers without lateral fringes; (9)

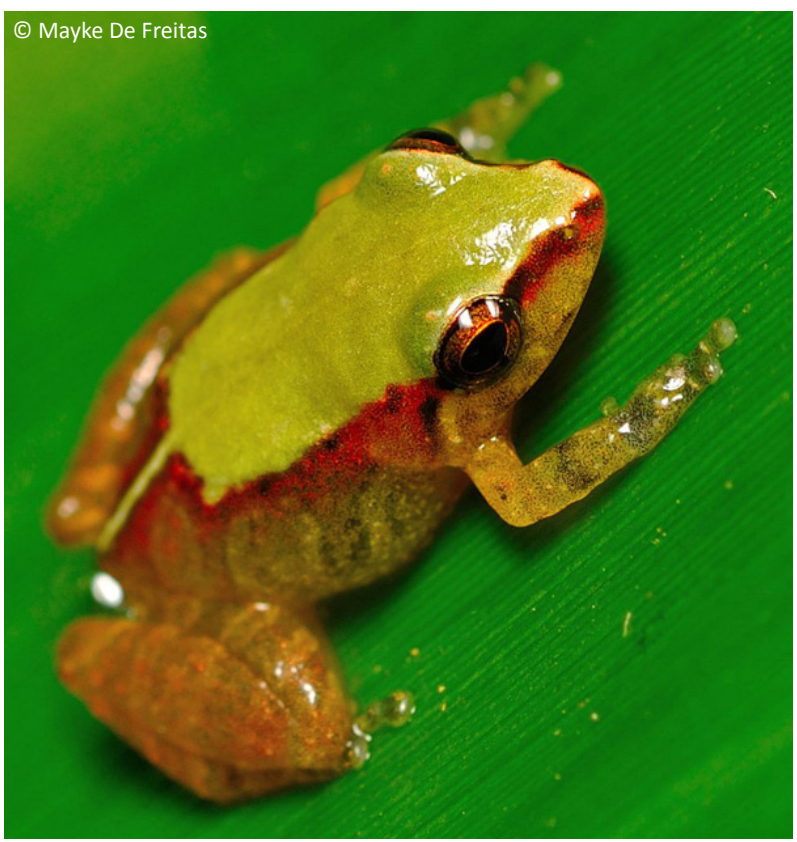

Image 17. Uncollected specimen of Pristimantis pariagnomus sp. nov. from Cerro Humo on the Península de Paria in northeastern Venezuela. Based on several characteristics, this individual can unequivocally be referred to the new species, particularly by the morphology of its fingers.
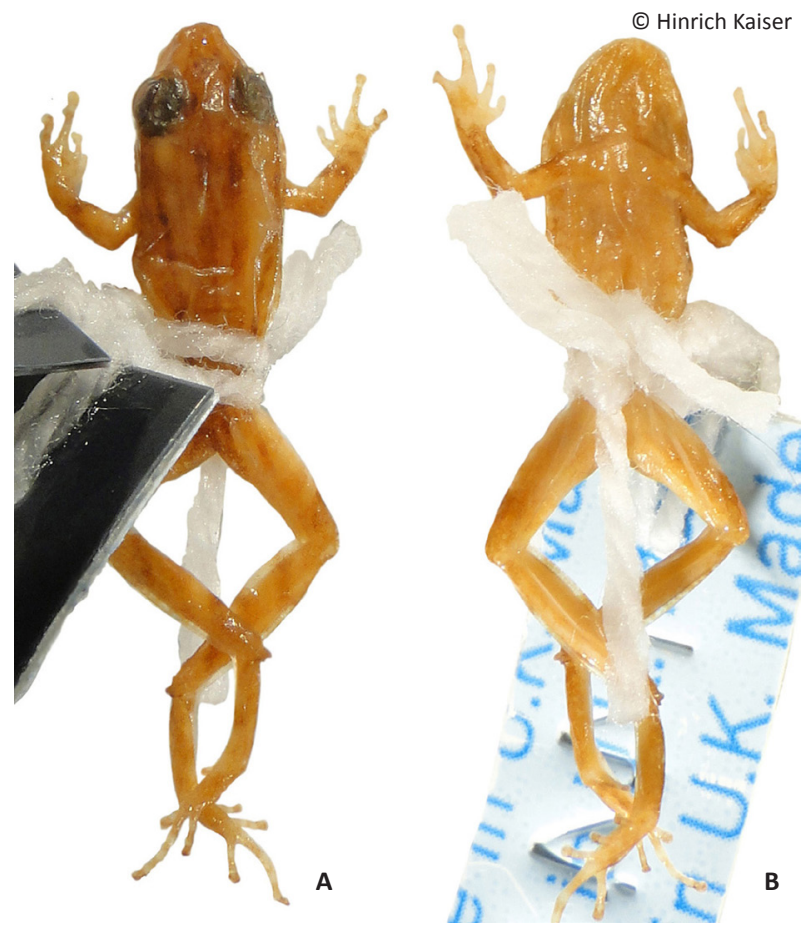

Image 18. Male holotype (MHNLS 14456, SVL $=13.0 \mathrm{~mm}$ ) of Pristimantis pariagnomus sp. nov. in dorsal (A) and ventral (B) views. 


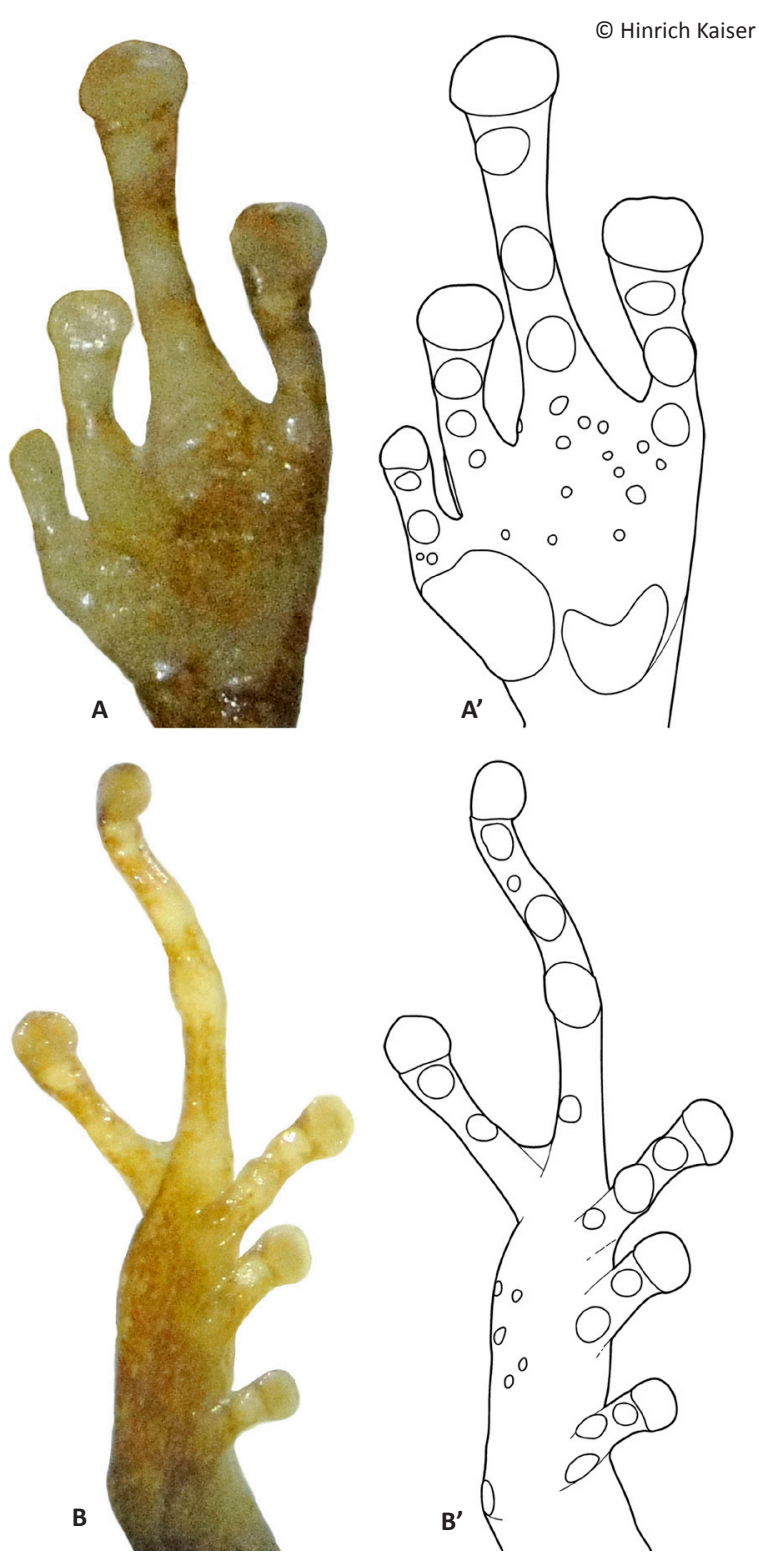

Image 19. Photographs and drawings of hand and foot of the holotype of Pristimantis pariagnomus sp. nov. (MHNLS 14456). (A $\left.A^{\prime}\right)$ Left hand. (B, B') Right foot.

many ulnar tubercles (Image 17), one raised tubercle on either side of the elbow; (10) prominent, protruding tarsal tubercles, with several additional low tubercles also present; prominent calcar tubercle present; inner tarsal fold absent; (11) two small metatarsal tubercles present; inner metatarsal tubercle flat and oval, half the size of the disk on $\mathrm{T}^{1}$; outer metatarsal tubercle small, round, about the same size of inner (Image 19B, $\mathrm{B}^{\prime}$ ); (12) size of toes $1=2<3<5<4$; toes without lateral fringes, remnant webbing present between $\mathrm{T}^{4}$ and $\mathrm{T}^{5}$; toes with only slightly expanded, oval disks; $T^{5}$ longer than $\mathrm{T}^{3}$, reaching the penultimate subarticular tubercle on $\mathrm{T}^{4} ;(13)$ color pattern light brown, with four indistinct darker longitudinal stripes, of which the two medial ones originate on the rostrum and the lateral ones originate at the posterior-most edge of the eye (the inverse pattern described as 'striped' by Lynch \& La Marca 1993), light brown venter.

\section{Comparisons}

Pristimantis pariagnomus is unique among coastal Cordillera Pristimantis in the following combination of characters: size very small (SVL of single specimen $13.0 \mathrm{~mm})$; tympanum minute, $14 \%$ of EYE; small dentigerous processes with only three vomerine teeth; reduced $\mathrm{F}^{1}$; prominent tarsal tubercle; very small metatarsal tubercles; disks on $\mathrm{F}^{1}$ and $\mathrm{T}^{1}$ very small; middorsal raphe absent, dorsolateral folds absent; cranial crests absent.

Pristimantis pariagnomus sp. nov. (characters in parentheses) is not similar to any of the species from northern Venezuela; herein the species is compared with those from the Venezuelan Cordillera de la Costa, Trinidad, and Tobago (see Table 2). Comparisons with $P$. nubisilva, $P$. hoogmoedi, $P$. geminus, and $P$. longicorpus are provided in the species accounts for those species. Pristimantis anotis is a much larger species, with females reaching $47 \mathrm{~mm}$ SVL (only known male $13 \mathrm{~mm}$ ), and it lacks a tympanum (indistinct tympanum present). Pristimantis bicumulus is also larger, with males reaching $23 \mathrm{~mm}$ SVL $(13 \mathrm{~mm})$, and has a round tympanum (oval), distinct canthus rostralis (ill-defined), oval dentigerous processes of the vomer (triangular) with 4-7 teeth (3 teeth), and areolate ventral skin (smooth). Pristimantis charlottevillensis is a Tobago endemic and a much larger species, with males reaching $31 \mathrm{~mm}$ SVL $(13 \mathrm{~mm}), \mathrm{F}^{1}>$ $F^{2}\left(F^{1}<F^{2}\right)$, a distinct tympanum (indistinct), presence of vocal slits (absent), and $\mathrm{T}^{5}<\mathrm{T}^{3}\left(\mathrm{~T}^{5}>\mathrm{T}^{3}\right)$. Pristimantis reticulatus is a larger species, with males up to $25 \mathrm{~mm}$ in SVL $(13.0 \mathrm{~mm})$, possessing a distinct, round tympanum (obscured, oval), a distinct canthus rostralis (ill-defined), a tongue without a medial notch (notched), pronounced differenced in metatarsal tubercles, and no webbing (webbing present between $\mathrm{T}^{4}$ and $\mathrm{T}^{5}$ ). Pristimantis riveroi is larger, with males reaching an SVL of $23 \mathrm{~mm}$ $(13 \mathrm{~mm})$, has coarsely areolate ventral skin (smooth), a distinct canthus rostralis (ill-defined), a tongue without a medial notch (notched), and a smaller outer metatarsal tubercle at $1 / 3$ the size of the inner (inner same size as outer). Pristimantis rozei is possibly a species of similar size, with the only known female specimen of $20 \mathrm{~mm}$ SVL $(13 \mathrm{~mm})$, but it has areolate ventral skin (smooth), a tympanum $1 / 3$ the size of the eye $(1 / 7$ the size of the 
eye), a distinct canthus rostralis (ill-defined), $F^{2}>F^{4}\left(F^{2}\right.$ $<\mathrm{F}^{4}$ ), and no webbing (webbing present between $\mathrm{T}^{4}$ and $\left.\mathrm{T}^{5}\right)$. Pristimantis stenodiscus is a similarly-sized species, with males up to $17 \mathrm{~mm}$ in SVL $(13 \mathrm{~mm})$, and with discs pointed at tips (rounded), a distinct canthus rostralis (illdefined), substantial differences in the morphology of metatarsal tubercles (Table 2), and no webbing (webbing present between $\mathrm{T}^{4}$ and $\mathrm{T}^{5}$ ). Pristimantis terraebolivaris is a much larger species, with males reaching $36 \mathrm{~mm}$ in SVL $(13 \mathrm{~mm})$, distinct, round tympanum (obscured, oval) with $2 / 3$ size of the eye $\left(1 / 7\right.$ size of the eye), $F^{4}>F^{2}\left(F^{2}>\right.$ $\mathrm{F}^{4}$ ), and no webbing (webbing present between $\mathrm{T}^{4}$ and $\mathrm{T}^{5}$ ). Pristimantis turpinorum, found only on Tobago, is a similarly-sized species, with males reaching $19 \mathrm{~mm}$ in SVL $(13 \mathrm{~mm})$, areolate ventral skin (smooth), round and distinct tympanum (oval, obscured), truncate snout in dorsal view (round), $F^{4}>F^{2}\left(F^{2}>F^{4}\right)$, and without tarsal tubercles (present). Pristimantis turumiquirensis, found only in the Turumiquire massif, is a much larger species, with females reaching $47 \mathrm{~mm}$ in SVL (the only male is $13 \mathrm{~mm}$ in SVL), round tympanum $1 / 2$ the size of the eye (oval, $1 / 7$ the size of the eye), distinct canthus rostralis (ill-defined), and no webbing (webbing present between $\mathrm{T}^{4}$ and $\mathrm{T}^{5}$ ). Lastly, $P$. urichi is a larger species, with males reaching $23 \mathrm{~mm}$ in SVL $(13 \mathrm{~mm})$, with a round tympanum (oval), distinct canthus rostralis (ill-defined), $F^{4}>F^{2}\left(F^{2}\right.$ $\left.>F^{4}\right), F^{3}=1.5 F^{1}\left(F^{3}=3.5 F^{1}\right)$, the presence of a pair of scapular tubercles (absent), and no webbing (webbing present between $\mathrm{T}^{4}$ and $\mathrm{T}^{5}$ ).

\section{Description of the holotype}

An adult male (based on epiphyseal fusion (Images $20 \mathrm{E}, 21 \mathrm{E}$ ) and observed vocalization; S. Gorzula, field notes), SVL $=13.0 \mathrm{~mm}$. Body moderately elongate; head narrower than body, $5 \%$ longer than wide; $\mathrm{HL} 42 \%$ of SVL; HW $37 \%$ of SVL. Snout rounded in dorsal view, truncate in profile; E-N $67 \%$ of EYE; nostrils slightly protuberant, directed laterally; canthus rostralis illdefined, rounded in cross section, concave in dorsal view; loreal region concave. Upper eyelid with a single conical tubercle, centered in the posterior half of the eyelid. Cranial crests absent. Tympanum obscured, oval, its horizontal diameter $14 \%$ of EYE, a tympanic annulus visible along its anterior half, with a weak, darkly colored supratympanic fold covering a small portion of its dorsal edge. Choanae small, ovoid, close to the maxillary arch, not concealed by palatal shelf; vomerine dentigerous processes small, triangular, widely separated, each bearing three teeth along their posterior edge, posterior and medial to choanae. Vocal slits absent. Tongue oval, slightly notched posteriorly, posterior half free.
Dorsal skin nearly smooth, with two parallel rows of tubercles; two postrictal tubercles present between tympanum and insertion of arm; middorsal raphe present; dorsolateral folds absent. Throat and chest areolate, belly and ventral surfaces of thighs areolate; flanks areolate inferiorly, posterior surfaces smooth.

Hand moderate in size, $29 \%$ of SVL. Relative lengths of adpressed fingers $1<4<2<3$; $F^{1}$ very reduced, when adpressed, $F^{1}$ reaching just past the proximal subarticular tubercle on $\mathrm{F}^{2}$ (Image 19A, $\mathrm{A}^{\prime}$ ). Finger disks rounded. Disk on $\mathrm{F}^{2}$ and $\mathrm{F}^{3} 1.6$ times wider than adjacent phalanx. Lateral fringes on fingers absent; webbing absent. Nuptial pads absent. Cordiform palmar tubercle and rounded, large, low thenar tubercle; thenar tubercle larger than palmar tubercle; subarticular tubercles, single, round, low, with the exception of the subarticular tubercle on $\mathrm{F}^{1}$, which are very small; several small supernumerary tubercles. Few low ulnar tubercles.

Hind limbs long; TIB 53\% of SVL (Table 4). Heel reaches the nostril when adpressed longitudinally to the body axis. Relative lengths of adpressed toes $1<2$ $<3<5<4$, neither $\mathrm{T}^{3}$ nor $\mathrm{T}^{5}$ reaching the penultimate subarticular tubercle on $\mathrm{T}^{4}$ (Image 19B, B'). Toes without lateral fringes, remnant webbing present between $\mathrm{T}^{4}$ and $\mathrm{T}^{5}$. Disks rounded, slightly wider than phalanges. Few tarsal tubercles present. One strongly developed conical calcar tubercle. Inner metatarsal tubercle small and oval; outer small and oval, of the same as the inner; subarticular tubercles distinct, single, round; a few indistinct supernumerary tubercles.

\section{Measurements of holotype (in $\mathrm{mm}$ )}

SVL: 13.0; TIB: 6.9; HL: 5.5; HW: 4.5; IND: 1.7; E-N: 1.4; EYE: 2.1; TYM: 0.3 wide, 0.6 high; $\mathrm{F}^{1} \mathrm{~L}: 1.2 ; \mathrm{F}^{2} \mathrm{~L}: 1.4$.

\section{Color in life}

This section is based on a photographic image of an individual that was not collected (Image 17). Based on the observable characteristics, it is clearly referable to $P$. pariagnomus. Dorsal background color greenishbrown, in this specimen overlaid with a very distinctive, uniform, light green coloration that extends from the tip of the snout across the first two-thirds of the dorsum and is framed by a reddish edge (Image 17). The reddish frame begins on the tip of the snout, runs along the canthus rostralis, is broken up by the eye, but continues on in the dorsolateral area until turning medially at a $90^{\circ}$ angle, then turning again to frame the middorsal raphe as it runs along in light green coloration to the cloaca. This is akin to the 'shirt and trousers' pattern we described for $P$. nubisilva, but with a light-colored 
'shirt' and darker 'trousers' and with the addition of the light-colored middorsal line. The light green dorsal color is pristine, and only the middorsal raphe is visible. The supratympanic strip is dark and boomerang-shaped and extends only a short distance from behind the eye before fading in coloration (Image 17). The flanks are greenish with some irregular brown patterning. The arms are greenish-brown with one faint crossbar on the lower arm. The thighs and shanks are a slightly darker greenish-brown, with darker brown crossbars. The upper lips are greenish-brown without any bars or spots. The iris is uniformly coppery brown with fine black reticulation.

\section{Color in preservative}

After over thirty years in $70 \%$ ethanol, the dorsal background color is uniformly brown, featuring four longitudinal darker brown stripes, of which the medial two are an extension of the dark brown snout area and the lateral two are an extension of the darker eyelid color; longitudinal stripes become confluent posteriorly and merge into the dark brown cloacal region; extremities also brown, arms with a single crossbar on the forearm; legs with crossbars, three each on femur and tibia, creating an effect that disrupts the shape of the leg and appears nearly aligned with the dorsal stripe pattern (Image 18A). Three dark brown labial bars, the widest one directly below the eye. Dark brown stripe bordering the canthus rostralis from the loreal region, not covering the canthus itself, but embracing the posterior edge of nares; supratympanic stripe narrow, dark brown, boomerang-shaped, covering upper edge of tympanum and turning behind tympanum towards the upper arm; two postrictal tubercles create a lighter brown pattern in the area between tympanum and upper arm. Ventrally, the coloration is uniformly brown, including the undersides of the limbs. Soles are more deeply pigmented than the top of the feet, whereas the palms of the hands are less pigmented than the back of the hands.

\section{Natural history}

The only preserved specimen of $P$. pariagnomus was collected along with a series of $P$. nubisilva and $P$. hoogmoedi in 1978. The field notes of Stefan Gorzula state that the "two smaller species" (i.e., P. nubisilva and $P$. pariagnomus) were collected "from low branches up the bank from the stream" where $P$. hoogmoedi were collected. The field notes also state that the collected specimens were calling males. Based on the overall size of $P$. pariagnomus, we consider it most likely to be a leaf- litter specialist, whose capture amongst a streamside chorus of $P$. nubisilva was coincidental. The photograph was taken of a single individual on a smooth-leaved plant near Cerro Humo. Two specimens referable to this species were observed in the leaf litter in a small open area in closed-canopy forest at an elevation of 1000 m on Cerro Humo in September 2014, and one of them was photographed (image 17).

\section{Distribution}

Only known from the southern slope of Cerro Humo in the Península de Paria. Its range is predicted to extend throughout the cloud forests at altitudes from 650-1200 m.

\section{Etymology}

The name pariagnomus is composed of two parts, the first part in reference to the geographic home of the species, the Península de Paria, and the second part derived from the Latin "gnomus" = dwarf, in reference to the very small body size of this species.

\section{OSTEOLOGY}

\section{Osteological comparisons}

Digital x-ray imaging makes available a data set for osteological comparisons that would otherwise necessitate invasive sampling (e.g., clearing and staining of whole specimens, preparation of skeletal material using dermestid beetles). In the particular case of the five species we describe herein, two ( $P$. longicorpus, $P$. pariagnomus) are represented by only a single specimen, whereas one other ( $P$. geminus) is represented by only two specimens. In none of these cases is invasive sampling an option. To ensure that our initial identification of five distinct taxa could be verified using an additional data set, we investigated the skull structure of the five species as well as the structure of hand bones. Our results show that there are indeed five distinct forms represented amongst the specimens we investigated, which we consider separate species. The $x$-ray analysis of $P$. pariagnomus was problematic because of the extreme small size of the specimen and the resulting loss of resolution. However, bone has clearly formed, with fainter areas in the digital images still showing a degree of ossification consistent with that of a miniaturized adult.

\section{Skull comparisons (Image 20)}

Rather than focusing on the individual structures of 


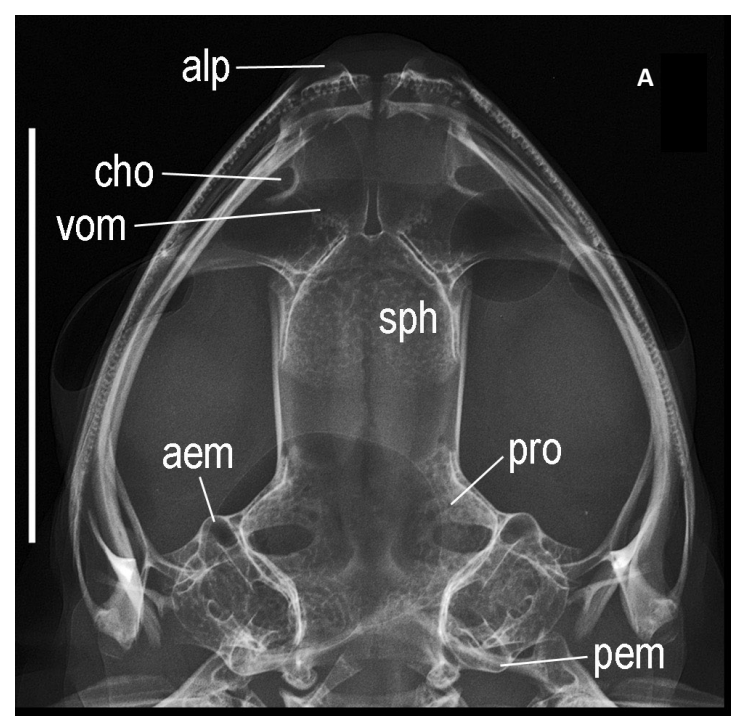

B
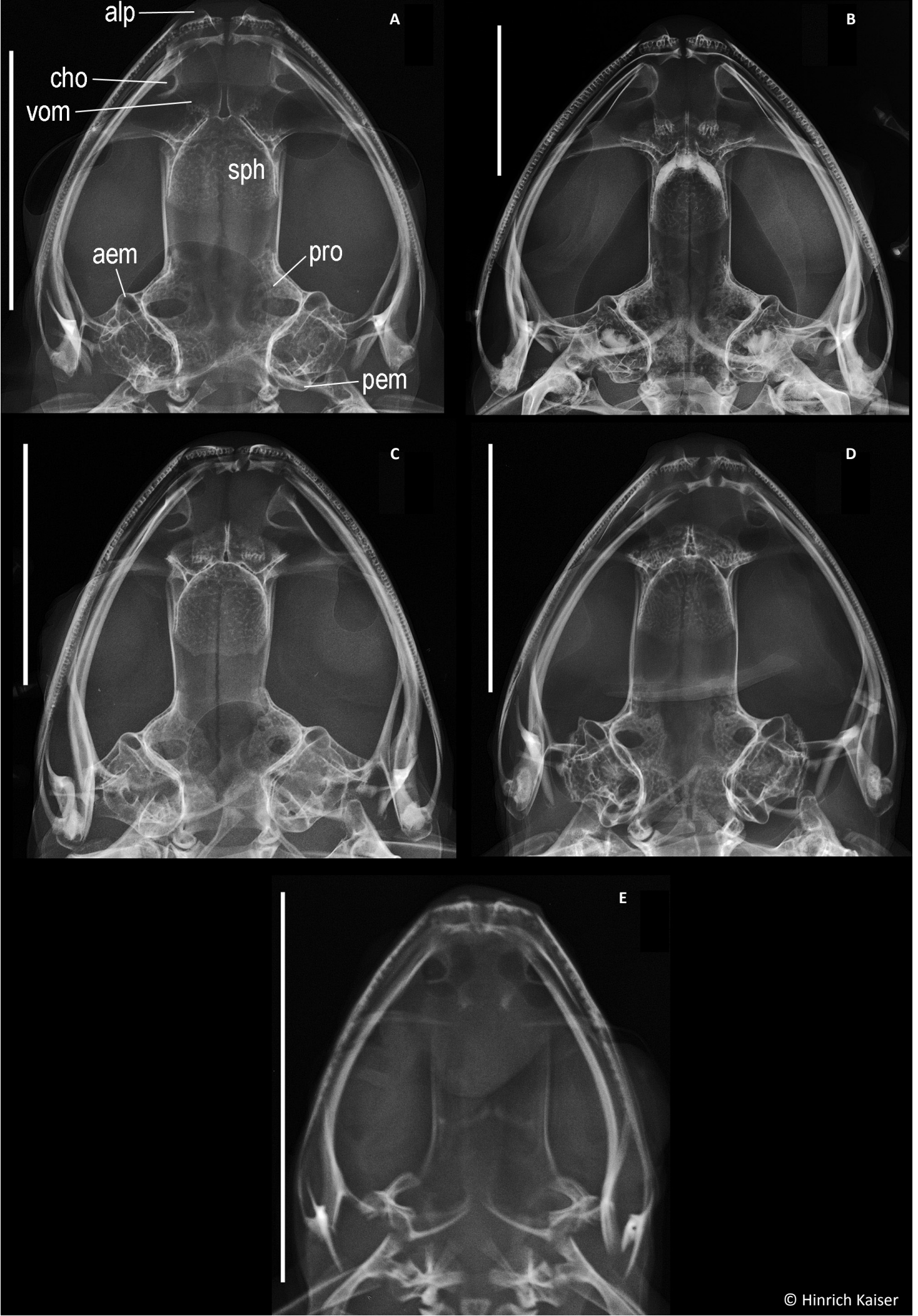

Image 20. Digital x-ray images of the crania of five species of Pristimantis from the Península de Paria in northeastern Venezuela. (A) P. nubisilva sp. nov. (CVULA 7424). (B) P. hoogmoedi sp. nov. (CVULA 7437). (C) P. geminus sp. nov. (USNM [CMD 341]). (D) P. longicorpus sp. nov. (CVULA 7444). (E) P. pariagnomus sp. nov. (MHNLS 14456). Abbreviations are provided in (A) for: alary process of the premaxilla (alp), choana (cho), vomer (vom), sphenethmoid (sph), anterior epiotic eminence (aem), posterior epiotic eminence (pem), and prootic (pro). Scale bars = $5 \mathrm{~mm}$. 
the skull, such as the shape or extent of specific bones, our analysis focuses on characters that would allow the most rapid comparison amongst species from a set of digital x-ray images. We believe that this approach has the greatest utility when analyzing an osteological data set for taxonomic purposes. While there is doubtlessly much information that could reveal evolutionary or developmental trends by a careful analysis of $\mathrm{x}$-rays, or even using more detailed analysis using CT scan imaging, we defer such an analysis to a study focused on cranial osteology.

The key components of the skull that readily lend themselves to recognition in the group of species we introduce here are the shape and position of choanae and dentigerous processes of the vomers, the number of premaxillary and maxillary teeth, the orientation of the alary process of the premaxilla, the width of the sphenethmoid, the transition from the sphenethmoid to the prootic (along the medial edge of the orbit), and the degree of protrusion of the anterior and posterior epiotic eminences. Cursory examination of the representative skulls for each of the five new species (Image 20) reveals a variety of slight differences in overall cranial construction. For the purposes of this discussion, we treat the most informative characters separately.

Alary process of the premaxilla: The alary process is an extension of the premaxilla that generally extends dorsally towards the nasal bone in support of prenasal cartilages. In some species, the process additionally displays an anterior element, which here occurs in all species with the exception of Pristimantis geminus.

Sphenethmoid: The sphenethmoid is a large bony element in the anterior section of the braincase. There are three characteristics of this bone that serve to distinguish amongst the five new species, the width of the sphenethmoid relative to skull width and the distance between the choanae, and the shape of its posterior margin. Pristimantis nubisilva (Image 20C) and $P$. pariagnomus (Image 20E) are categorized as possessing a wide sphenethmoid, with a width exceeding $30 \%$ of the skull width. In contrast, $P$. hoogmoedi has a narrow sphenethmoid with a width of just less than $20 \%$ of skull width (Image 20B). Both $P$. geminus and $P$. longicorpus have intermediate values of $24 \%$ and $26 \%$, respectively. The distance between choanae relative to sphenethmoid width also provides a means to differentiate these skulls. Whereas the choanal distance in $P$. longicorpus is equal to the sphenethmoid width (Image 20D), in P. nubisilva and $P$. geminus it is ca. $20 \%$ shorter (choanal distances of $83 \%$ and $79 \%$, respectively), and in $P$. hoogmoedi and $P$. pariagnomus it is over $30 \%$ shorter (choanal distances of
$67 \%$ and $69 \%$, respectively). The posterior margin of the sphenethmoid is curved into a U-shape in $P$. nubisilva, $P$. hoogmoedi, and $P$. geminus, whereas it is straight in $P$. longicorpus and $P$. pariagnomus.

Choanae: The shape and position of anuran choanae, openings connecting the nasal cavity with the mouth, are frequently used diagnostic characters because even though they are a skeletal element they are visible in the open mouth. In the frog species under investigation, the medial margin of the choanae of $P$. nubisilva and $P$. longicorpus is rounded (more deeply concave), whereas in the other three species it is more ovoid (a more open curve). The position of the choanae with respect to the dentigerous processes of the vomer is the second feature of note. In $P$. hoogmoedi and $P$. geminus, the edges of the choanae are so close together as to sit more medially in the palatal region than the most lateral extent of the dentigerous processes. In the other species, the choanae are more widely separated.

Dentigerous process of the vomer: In the genus Pristimantis, the dentigerous processes of the vomers are palatal tooth-bearing structures thought to aid in prey handling and swallowing. All five species have differently shaped dentigerous processes, generally in a triangular fashion, bearing different tooth numbers ranging from three teeth per process in $P$. pariagnomus to nine in $P$. longicorpus.

Premaxillary and maxillary teeth: Numbers of premaxillary and maxillary teeth vary widely among the five species under investigation. Pristimantis pariagnomus stands out by having only eight premaxillary and 25 maxillary teeth, and these low numbers may be a result of miniaturization. The other species have 10-15 premaxillary teeth, with the larger numbers $(14,15)$ found only in $P$. hoogmoedi. It may be surprising that the largest species, $P$. hoogmoedi, presents with the lowest number of maxillary teeth (52-58) with the exception of the miniaturized $P$. pariagnomus. Maxillary tooth numbers among the other three species are non-overlapping, with 74-82 ( $P$. nubisilva), 72 ( $P$. longicorpus), and 65-69 ( $P$. geminus) in descending order.

Medial edge of the orbit: The medial edge of the orbit is a transition zone in which the anterior elements of the rostrum transition to the braincase. In the five species discussed here, this area is distinctly shaped into an angular section (in $P$. nubisilva, $P$. hoogmoedi, and $P$. geminus) or a rounded area (in $P$. longicorpus and $P$. pariagnomus).

Anterior and posterior epiotic eminences: The anterior and posterior epiotic eminences are bony 


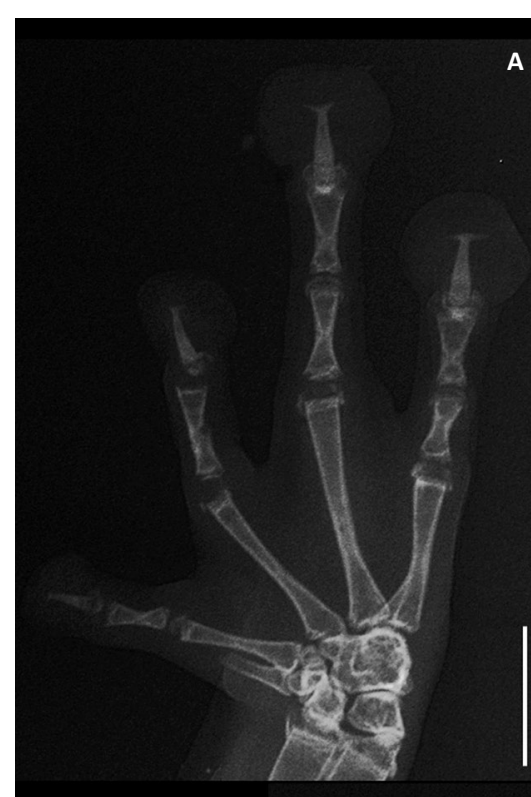

A

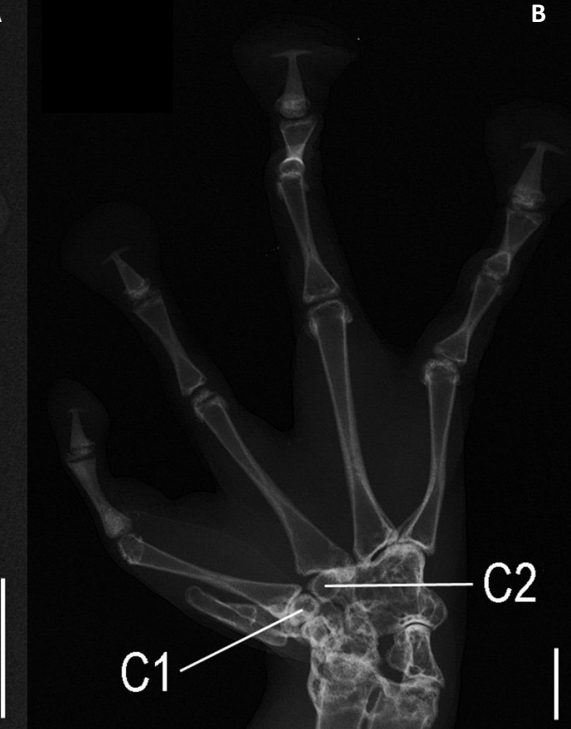

D

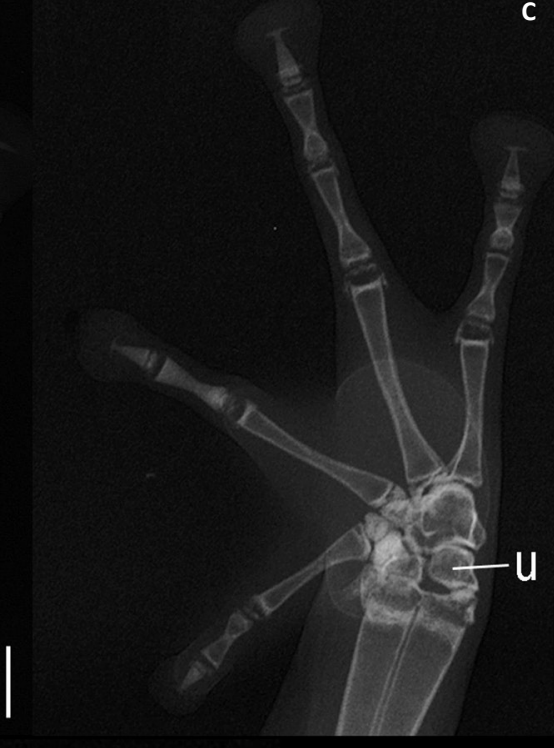

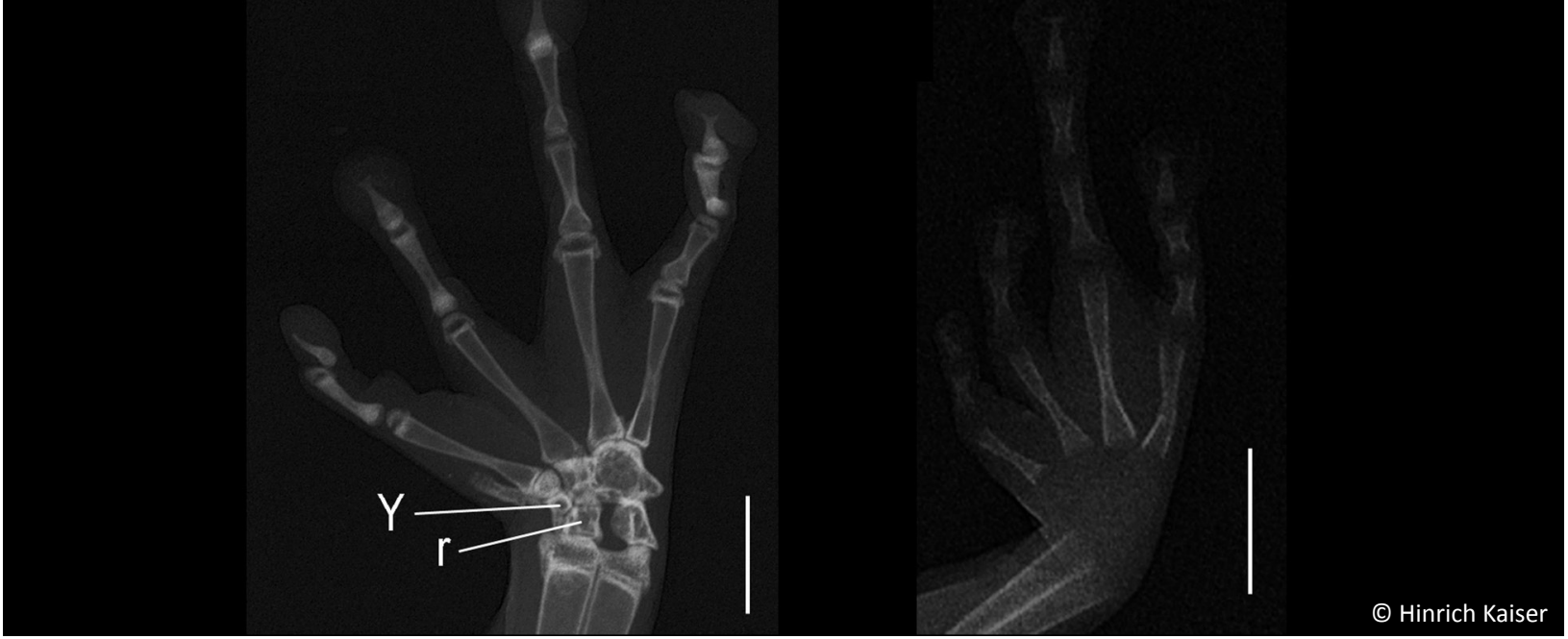

Image 21. Digital x-ray images of the hands of five species of Pristimantis from the Península de Paria in northeastern Venezuela. (A) P. nubisilva sp. nov. (CVULA 7425). (B) P. hoogmoedi sp. nov. (CVULA 7435). (C) P. geminus sp. nov. (USNM [CMD 341]). (D) P. longicorpus sp. nov. (CVULA 7444). (E) P. pariagnomus sp. nov. (MHNLS 14456). Abbreviations are provided in (B) for carpal 1 (C1) and carpal 2 (C2), in (C) for ulnare (u), in (D) for Element $Y(Y)$ and radiale $(r)$. Scale bars $=1 \mathrm{~mm}$.

ridges resulting from the fusion of the prootic and the exoccipital bones as they form the anuran braincase and the back of the braincase. In the five species under investigation, the anterior epiotic eminence invariably protrudes into the orbital space, to different degrees. Most prominent is the situation in $P$. longicorpus, where the incursion is not merely a bony nub but a protrusion. Similarly, in that species as well as in P. pariagnomus the posterior epiotic eminence protrudes posteriorly from the cranium in a pronounced, condyle-like fashion.

\section{Hand comparisons (Image 21)}

Key elements of the hand relevant to species distinction include mesopodial (ulnare, radiale, Element $\mathrm{Y}$ ) and metapodial (metacarpals) elements in addition to the shape of the terminal phalanges. We have considered the ratios of several phalangeal and metacarpal measurements (Table 3) and find them largely uninformative due to overlap. However, there are several key differences in terms of gross osteological features.

Mesopodial elements: Whereas the radiale in $P$. geminus and $P$. hoogmoedi is a nearly round bone, 
it is approximately rectangular in $P$. nubisilva and $P$. longicorpus. There is a well-developed lateral process on the proximal edge of the ulnare in $P$. hoogmoedi, $P$. longicorpus, and $P$. geminus that is not developed in $P$. nubisilva. Element $Y$ has a proximal extension in $P$. longicorpus and $P$. geminus that is absent in $P$. nubisilva and $P$. hoogmoedi. Carpal 1 is much reduced in $P$. nubisilva, whereas in $P$. longicorpus it is nearly rectangular and twice as large as in the other species. The mesopodium and skull of $P$. pariagnomus are not well ossified.

Metapodial and phalangeal elements: The key difference in terms of phalangeal morphology lies with the lateral expansion of the phalangeal termini. In three of the species ( $P$. geminus, $P$. hoogmoedi, $P$. pariagnomus), these are T-shaped, whereas in $P$. longicorpus they are Y-shaped. Pristimantis nubisilva presents an anomaly, with a mosaic of T- and Y-shaped termini. Among 128 termini examined, 88 were $Y$-shaped (69\%). T-shaped termini occurred with different frequencies on the phalanges $\left(F^{1}-2, F^{2}-10, F^{3}-10, F^{4}-18\right)$, and there is no predictability of termini even in single individuals: MNHLS 14465 has a T-shaped terminus on $\mathrm{F}^{3}$ of the left hand but not on the right hand, and the termini of $\mathrm{F}^{4}$ on both hands are T-shaped.

\section{DISCUSSION}

Nearly two decades ago, Kaiser (1994) predicted that the absence of Pristimantis-like forms in the mountains of the Península de Paria was likely an artifact of limited herpetological research. Given the presence of endemic Pristimantis in the northern range of Trinidad ( $P$. urichi) and the Main Ridge of Tobago ( $P$. charlottevillensis and $P$. turpinorum, the latter at that time considered Eleutherodactylus cf. rozei) as well as endemic Mannophryne in the same mountains ( $M$. trinitatis on Trinidad and M. olmonae on Tobago), the senior author reasoned (Kaiser 1994, 1996) that the diversity gap for these types of frogs would eventually be filled in and the biogeographic link between the mountainous ridges on Tobago and Trinidad and those of northeastern Venezuela (Frost \& Snoke 1989) would be supported by whichever species filled the gap. Little did these authors know that material of several new species of Pristimantis had already been collected in 1978 and deposited in two herpetological collections. Unfortunately, access to collection information was limited at that time and it was not practical to cast a wide net looking for the existence of specimens within the time limits of the research project. Thus, the senior author only rediscovered these specimens in 2001 during a visit to Venezuela, during which the new material was collected.

\section{Taxonomy}

We consider the presentation of taxonomic decisions, in this case covering five new species, a very serious matter that should never be rushed or taken lightly. Whereas two of the five species, $P$. nubisilva and $P$. hoogmoedi, are represented by specimen series that allowed a description of the variation within each taxon as well as by chromosomal data, the sample size for $P$. geminus is only two, and $P$. longicorpus and $P$. pariagnomus are represented in collections by only single specimens. In terms of evidence, a stronger case can generally be made for describing species of which many specimens are available. Under what circumstances is it therefore advisable to proceed with a formal taxonomic decision even when there are only very few specimens available?

In many disciplines of zoology, particular in paleontology and zoological deep-sea oceanography, is the availability of large sample sizes the exception rather than the rule. However, when descriptions are carefully crafted, important knowledge is certainly gleaned from single specimens, will allow comparisons with other forms, and, if clear-cut morphological differences can be demonstrated, availability of a taxon name simplifies the integration of the rare forms into the scientific discourse. In some instances this integration becomes possible via the use of molecular data, which in this case could not be done. We therefore tried to compensate by using $x$-ray data in addition to the more traditional external morphological data, which provided additional characteristics.

Even though we struggled with the decision to formally name $P$. longicorpus and $P$. pariagnomus, the two species for which only a single specimen exists, both forms possess unique suites of characteristics that permit their easy differentiation from other species in the genus Pristimantis. In addition, ongoing contemporaneous conservation efforts in the Península de Paria necessitate that biodiversity data are as complete as possible to ensure the greatest impact of these efforts. Thus, naming taxa is a more significant statement regarding diversity than flagging a population as a candidate species.

\section{Diversity and biogeography}

With the recognition of these five new species, the diversity of Pristimantis in the Península de Paria is 
now more in line with what would be expected from a mainland fauna vis-à-vis the island faunas of Trinidad and Tobago, according to the standard views on island biogeography, according to which there should be a reduction of diversity from mainland to recent islands (MacArthur \& Wilson 1967). The gap in the diversity of frogs in the Península de Paria began to be filled in by the descriptions of Mannophryne riveroi (DonosoBarros 1964) and M. venezuelensis (Manzanilla et al. 2007), which closed it for Mannophryne. A further dendrobatid was described by Barrio-Amorós et al. (2006) as a member of the genus Colostethus, but it is now known as Allobates caribe (Barrio-Amorós et al., 2006). The presence of three centrolenid frogs, Celsiella vozmedianoi (Ayarzagüena \& Señaris 1996), and Vitreorana castroviejoi (Ayarzagüena \& Señaris 1996) filled in the gap in glass frogs between the Venezuelan mountains and Tobago, where Hyalinobatrachium orientale tobaogense (Hardy 1984; see Jowers et al. 2014 for validation of the subspecific status) is endemic. The five species of Pristimantis we described here not only fill in the diversity gap illustrated by Hedges et al. (2008: Image 115), but based on their morphology, they may also represent phylogenetic links with the species in Trinidad and Tobago and the central sector of the Venezuelan coastal mountain chain. For example, both $P$. nubisilva and $P$. geminus look remarkably similar to their island neighbors. Pristimantis turpinorum has an external phenotype that is strikingly similar to that of $P$. nubisilva, whereas $P$. geminus truly looks like a darker brown twin to $P$. urichi (Image 12B), down to the two dark scapular spots. Furthermore, $P$. hoogmoedi is quite similar to $P$. anotis from Rancho Grande, Estado Aragua, Venezuela, in gross morphology. These two species can be easily separated by the presence of a tympanum in $P$. hoogmoedi (lacking in $P$. anotis), but both have similar size and habitus. We find it worth noting that to date we have not found any representatives of the $P$. conspicillatus group (of which $P$. terraebolivaris from the Cordillera de la Costa and $P$. charlottevillensis from Tobago are members; Hedges et al. 2008) on the Península de Paria.

\section{Chromosomes and species groups}

Chromosome characteristics are an interesting data set for comparisons, but these are unfortunately not available for all the taxa under investigation. Based on the massive data set of Schmid et al. (2010) there is considerable variability in chromosome number and morphology, which is not easily reconciled with biogeographic and phylogenetic models at this time.
The taxa in our investigation are a microcosm of this challenge. Whereas the Pristimantis unistrigatus species group, to which we have assigned four of the five new species, has been considered a morphologically cohesive unit (Lynch \& Duellman 1997), Hedges et al. (2008) demonstrated that it was not a natural group but a grab-bag for species that could not be assigned to other, confirmed monophyletic groups. Among the species we investigate here, several fundamental numbers (FN) of chromosomes are represented. Pristimantis unistrigatus and $P$. hoogmoedi both have $\mathrm{FN}=34$, whereas $P$. nubisilva and $P$. riveroi have $\mathrm{FN}=$ 36. On Trinidad and Tobago (P. turpinorum, P. urichi), this number changes to $\mathrm{FN}=32$.

\section{Miniaturization}

Pristimantis pariagnomus may be the smallest frog species in Venezuela and rank among the smallest in South America. Miniaturization is not unusual in the genus Pristimantis, as outlined in the discussions by Terán-Valdez \& Guayasamin (2010) and Lehr \& Coloma (2008). Extremely small species include $P$. andinognomus Lehr \& Coloma 2008, P. caeruleonotus Lehr et al. 2007, P. carvalhoi (Lutz 1952), P. imitatrix (Duellman 1978), $P$. minimus Terán-Valdez \& Guayasamin 2010, and $P$. trachyblepharis (Boulenger 1918), and these have a distribution associated with the Andes and adjacent Amazonian lowlands. As with miniaturization in other amphibians (e.g., frogs: Hanken \& Wake 1993; salamanders: Hanken 1982, Hanken \& Wake 1994), the process affects skeletal morphology. As a consequence of miniaturization, loss of phalangeal and cranial elements has been reported as an extreme feature, whereas reduction in ossification or mineralization appears to be more common in terraranan frogs, such as Pristimantis (Yeh 2002). In P. pariagnomus, there appears to be no loss of elements, in concordance with observation in other miniaturized species of the genus (e.g., Terán-Valdez \& Guayasamin 2010), but there is reduced ossification. Based on a single specimen and the observation of one living individual and the size variation reported in other small species (e.g., 17.1-21.3 $\mathrm{mm}$ in P. gryllus; Barrio-Amorós et al. 2012), we expect that the size range of males may exceed $13 \mathrm{~mm}$.

\section{Natural history}

The description of five distinct species in a very restricted area on Cerro El Humo raises the question of how these are able to divide the habitat and limit the potential for competition. We note that based on the limited collecting effort to capture specimens, there 
appears to be a difference in abundance of these species. Given an equal effort throughout the time of collection, with the exception of the bromeliad-dwelling $P$. geminus, which would require special attention to bromeliads, each individual had about the same chance of being captured; this indicates that the most frequently seen species were probably the most abundant in the habitat. By this reasoning, $P$. nubisilva and $P$. hoogmoedi are expected to be the most abundant of the five species. The other three species are known from fewer specimens, and they should therefore be considered less abundant overall. Pristimantis hoogmoedi appears to be a habitat specialist that occupies rock walls in and along fast-flowing streams. It is therefore likely not directly in competition with the other four species, with the exception of stream edges. The miniaturized $P$. pariagnomus is able to exploit the layers of the leaf litter, which are inaccessible to the larger species. By their overall body morphology, including limb lengths and disk expansion, we expect $P$. nubisilva and $P$. longicorpus to occupy low branches of bushes as well as the ground vegetation. These species are therefore likely to experience the effects of competition. However, the unusual, elongated body shape of $P$. longicorpus may represent an adaptation to some as yet unidentified microhabitat. Kaiser (1993, 1997) noted the unusually short limbs among individuals of Eleutherodactylus johnstonei on the xeric island of Barbuda and proposed that this statistically significant reduction in limb length may be an adaptive response to life in water cisterns, the only locations on that island with suitable moisture. Thus, some unknown force of selection may also be at the root of limb reduction in $P$. longicorpus. The greater abundance of $P$. nubisilva points to the fact that this species might be better adapted for the particular habitat surveyed, or act as a generalist, whereas $P$. longicorpus may be more common in other, as yet unknown, microhabitat situations. Lastly, we only found $P$. geminus in bromeliads collected from high on trees; in an effort limited to ten bromeliads, we found two specimens.

\section{Conservation}

Based on our limited experience with the five species we describe here, we offer our recommendations for assignment of IUCN Red List categories (IUCN 2001) as follows. Pristimantis nubisilva was locally very abundant, and chorusing indicated the presence of many individuals throughout the habitat. We tentatively consider it a species that may be categorized as Least Concern (LC), recognizing that local abundance combined with geographic restriction may indicate that this species may need to be assigned to a different category as more research is done. Conversely, $P$. hoogmoedi, while also relatively abundant locally, was much more restricted in terms of its microhabitat (streams). One of us (GAR), however, has conducted research in the region during 2013 and 2014, and did not find the species at localities where a decade ago it was common. The dependence of this species on small water courses, combined with its limited range in the higher elevations of the Península de Paria, suggests that a status of Vulnerable (VU) might be appropriate for this species. Given the small number of specimens assigned to $P$. geminus, $P$. longicorpus, and $P$. pariagnomus, we believe these species should be listed as Data Deficient (DD) pending further surveys.

The Península de Paria can be recognized as one of the herpetologically most diverse regions of Venezuela. That is consistent with its status as a Dispersal Center, Plant Refuge, and Center of Endemism, all related to the relict element of its cloud forests (Steyermark 1979). Officially, most of the cloud forests in the peninsula are under legal protection because they are situated within the boundaries of the Parque Nacional Península de Paria. However, given the environmental continuity of the habitat and the artificial boundaries of the national park, important isolated mountains scattered throughout the peninsula, such as El Paujil, Campeare, or Cerro La Cerbatana, where new discoveries of plant taxa have taken place recently (Grande \& Meier 2012), reliance on the establishment of a bureaucratic construct for conservation is problematic.

While the national park was created in 1979 , it has never been properly funded or managed, lacking both zoning plans and enforceable rules of use. Two park rangers are charged with covering a wilderness area of $37,500 \mathrm{ha}$, with just one ranger guard post and almost no equipment. Deforestation, selective logging, and hunting are rife and, although illegal, commonly practiced within the park. Human-caused forest fires are a significant threat, even in the most isolated areas, such as Cerro Patao, where several thousand hectares of lowland semi-deciduous dry forest have been transformed into savannah habitats as a result of "conuco" agriculture involving slash-and-burn practices. In addition, ca. $60 \%$ of riparian and $40 \%$ of evergreen forests have been converted into coffee or cocoa plantations. However, despite of relatively high recent cocoa prices, most plantations are abandoned or low-producing, creating a reliance of local farmers on cash crops.

A massive liquefied gas project is increasing immigration and urban encroachment, putting even more pressure on the park's resources. The state 
Key to 16 species of Pristimantis from the northern mountain range crossing Venezuela and into Trinidad and Tobago

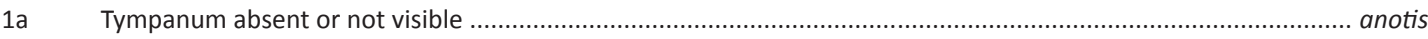

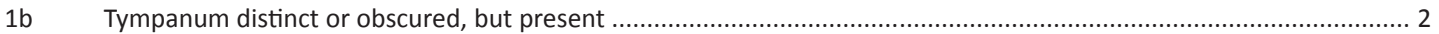

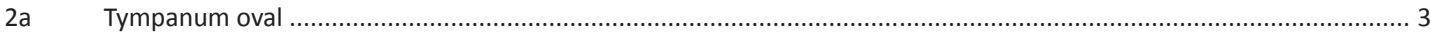

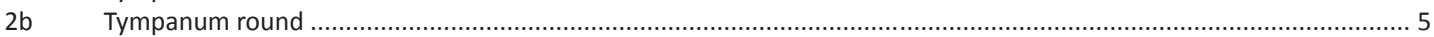

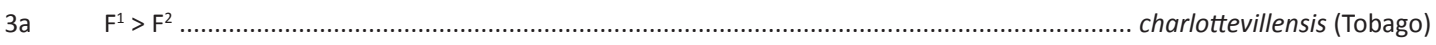

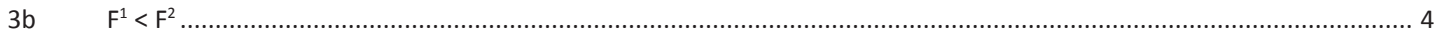

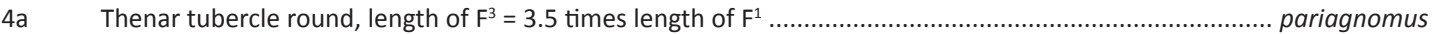

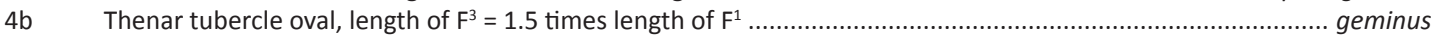

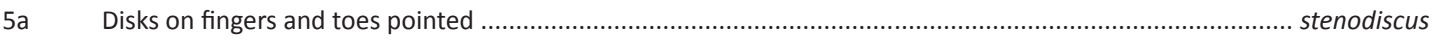

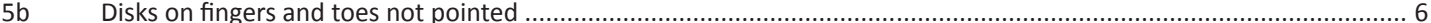

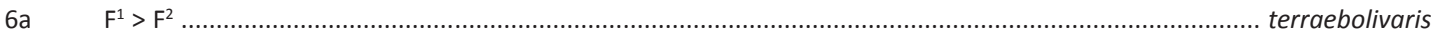

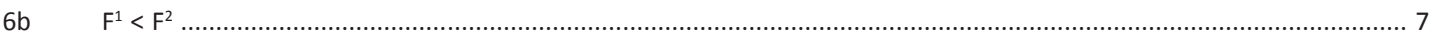

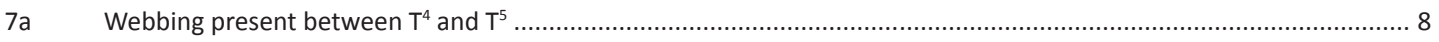

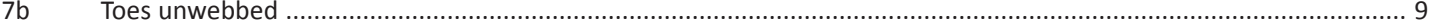

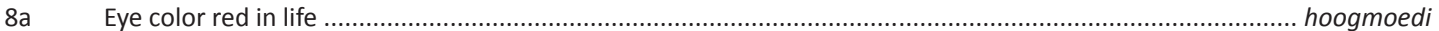

$8 \mathrm{~b} \quad$ Eye color metallic brown ........................................................................................................ turpinorum (Tobago)

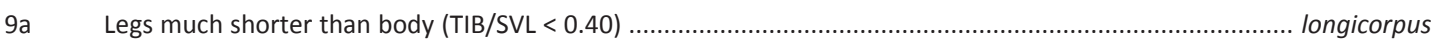

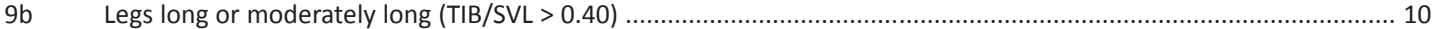

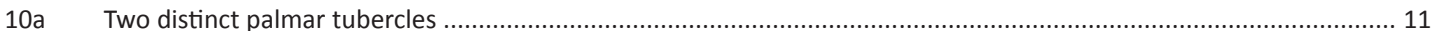

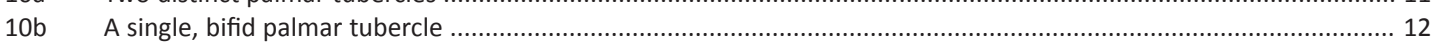

11a Adpressed $\mathrm{T}^{5}$ barely reaches the penultimate subarticular tubercle on $\mathrm{T}^{4}$...................................................nubisilva

11b Adpressed $\mathrm{T}^{5}$ surpasses the penultimate subarticular tubercle on $\mathrm{T}^{4}$............................................... urichi (Trinidad)

12a With an enlarged, protuberant calcar tubercle .......................................................................................... bicumulus

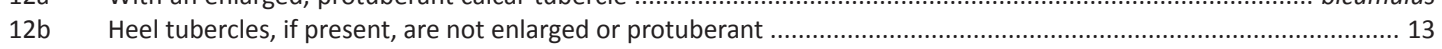

13a Posterior surfaces of thighs with small white spots ....................................................................................... reticulatus

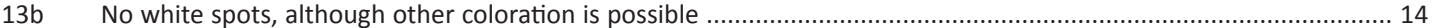

14a Outer metatarsal tubercle absent ........................................................................................................ turumiquirensis

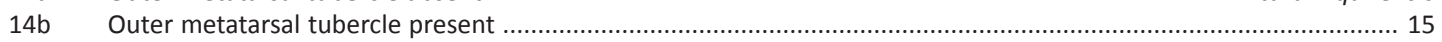

15a Brown body color with orange brown thighs in life, thin dark crossbars on the hind limbs ..................................... rozei

15b Grey with marbled brown thighs in life, dark and light crossbars of equal width .............................................. riveroi

owned oil company PDVSA has pledged to re-open the Güiria-Macuro road and a gypsum mine in the area to increase mobility and jobs for locals. In contrast, we believe that better road access could instead spread deforestation into pristine areas of the park, such as Cerro Patao, Cerro Azul, and Cerro El Olvido. The demographics of the Paria Peninsula show that the liquefied gas plant and related projects will have to rely on an enormous amount of skilled and unskilled labour unavailable locally, therefore driving up immigration into the area, and reducing the socioeconomic benefits for the local population. Lack of enforcement and surveillance exacerbate and undermine the already poor performance of the national park as a conservation entity. Although impoverished, situation of most of the rural population is the biggest threat to the integrity of the park, an almost complete absence of investment into research and conservation is a most pressing issue (Mayke De Freitas, pers. comm. 9 April 2014). We hope that the addition of five new and putatively endemic frog species will add some more weight to the natural importance of the Península de Paria, both with respect to biodiversity and conservation as well as its unique position to serve as an ecotourism destination, just as is being developed in neighboring Trinidad \& Tobago.

\section{Appendix 1. Comparative Material}

Pristimantis anotis $(\mathrm{n}=1)$ : VENEZUELA: Estado Aragua, Rancho Grande, UMMZ 109876 (holotype).

Pristimantis bicumulus $(n=10)$ : VENEZUELA: Distrito Federal, Caracas, ZMB 4899 (two syntypes), AMNH 81458-59; Distrito Federal, El Junquito, USNM 12080204; Estado Aragua, km 29 of the Maracay-Ocumare de la Costa road, KU 185678-80.

Pristimantis charlottevillensis $(n=36)$ : TOBAGO: Hills above Man-of-War Bay, 1.5-3.5 km ENE Charlotteville, AMNH 87408, 87412, 87427-28, 87431; Parish of St. John, mile marker $273 / 4$ on Charlotteville-Bloody Bay Road, USNM 167609-11, 167613; ca. 7km N Roxborough, KU 222409 (holotype), NMC 35056, 35060-1-5, 350641-3, 35064-5-11, KU 222381; 1.5km SW Charlotteville, KU 222370-80, 222410; Kings Bay Waterworks, ZMB 
53746-47.

Pristimantis reticulatus ( $\mathrm{n}=5$ ): VENEZUELA: Estado Aragua, Rancho Grande, Pico Periquito, UMMZ 109872 (holotype); Estado Aragua, $29 \mathrm{~km}$ of the MaracayOcumare de la Costa road, KU 185674-76, 185681.

Pristimantis riveroi $(n=16)$ : VENEZUELA: Estado Aragua, Rancho Grande, AMNH 70599 (holotype), 70600-611, KU 166385-86, 185683.

Pristimantis rozei $(\mathrm{n}=1)$ : VENEZUELA: Estado Aragua, Curucuruma, MBUCV 2018 (holotype).

Pristimantis stenodiscus ( $n=1)$ : VENEZUELA: Estado Aragua, Rancho Grande, Pico Periquito, UMMZ 109886 (holotype).

Pristimantis terraebolivaris $(n=26)$ : VENEZUELA: Estado Aragua, Rancho Grande, MCZ

31062 (holotype), UMMZ 113950, 113951 (two specimens), 113952-54, 113955 (four specimens), 113956, 113957 (two specimens), 113958 (two specimens), 113960-64; Miranda State, Los Canales, Planta Electrica de Naiguata, USNM 128807-08, 128812-14 (paratypes).

Pristimantis turpinorum $(n=4)$ : TOBAGO: Parish of St. John, mile marker $273 / 4$ on Charlotteville-Bloody Bay road, MCZ 86950, 86952-53, USNM 227815 (holotype).

Pristimantis turumiquirensis $(n=3)$ : VENEZUELA: Estado Monagas, Pico Turumiquire, AMNH 22557 (holotype), 22558-59 (paratypes).

Pristimantis urichi $(\mathrm{n}=24)$ : TOBAGO: Main Ridge, ca. $7 \mathrm{~km}$ N Roxborough, NMC 35059, 350631-32, KU 265456. TRINIDAD: N Arima Valley, NMC 35057-1-2, 35061-1-8, 35065-1-5, KU 265457-58, KU 222382; no further locality, SMF 3818 (lectotype).

\section{REFERENCES}

ASIH (American Society of Ichthyologists and Herpetologists) (2004) Guidelines for Use of Live Amphibians and Reptiles in Field and Laboratory Research, $2^{\text {nd }}$ Edition. ASIH, Lawrence, Kansas, USA.

Ayarzagüena, J. \& C. Señaris (1996). Dos nuevas especies de Cochranella (Anura; Centrolenidae) para Venezuela. Publicaciones de la Asociación de Amigos de Doñana 8: 1-16.

Barrio-Amorós, C.L. (1998). Sistemática y biogeografía de los anfibios (Amphibia) de Venezuela. Acta Biologica Venezuelica 18(2): 1-93.

Barrio-Amorós, C.L. \& H. Kaiser (2008). Distribution of Strabomantis biporcatus (Terrarana: Strabomantidae) in northern Venezuela, with comments on its phenotypic variation. Salamandra 44: 248254.

Barrio-Amorós, C.L., G. Rivas \& H. Kaiser (2006). New species of Colostethus (Anura, Dendrobatidae) from the Península de Paria, Venezuela. Journal of Herpetology 40: 371-377; http://dx.doi. org/10.1670/0022-1511(2006)40[371:NSOCAD]2.0.CO;2

Barrio-Amorós, C.L., G.A. Rivas, C. Molina, J.C. Santos \& H. Kaiser (2010a). Intraspecific variation in the endangered frog Mannophryne riveroi (Anura, Dendrobatidae, Aromobatinae), with comments on coloration and natural history. Herpetology Notes 3: 151-160.
Barrio-Amorós, C.L., F.J.M. Rojas-Runjaic \& T.R. Barros (2010b). Two new Pristimantis (Anura: Terrarana: Strabomantidae) from the Sierra de Perijá, Venezuela. Zootaxa 2329: 1-21.

Barrio-Amorós, C.L., J.M. Guayasamin \& S.B. Hedges (2012). A new minute Andean Pristimantis (Anura: Strabomantidae) from Venezuela. Phyllomedusa 11: 83-93.

Barrio-Amorós, C.L., M.P. Heinicke \& S.B. Hedges (2013). A new tuberculated Pristimantis (Anura, Terrarana, Strabomantidae) from the Venezuelan Andes, redescription of Pristimantis pleurostriatus, and variation within Pristimantis vanadisae. Zootaxa 3647(1): 4362.

Donoso-Barros, R. (1965a). Nuevos reptiles y anfibios de Venezuela. Noticiario Mensual, Museo Nacional de Historia Natural, Santiago, Chile 102: 2-3.

Donoso-Barros, R. (1965b). A new dendrobatidae [sic] frog, Prostherapis riveroi from Venezuela. Caribbean Journal of Science 4: 485-489.

Donoso-Barros, R. (1966). Dos nuevos Gonatodes de Venezuela. Publicación Ocasional del Museo Nacional de Historia Natural, Santiago de Chile 11: 1-32.

Edwards, S.R. (1974). Taxonomic notes on South American dendrobatid frogs of the genus Colostethus. Occasional Papers of the Museum of Natural History, University of Kansas 30: 1-14.

Evenhuis, N.L. (2008). A compendium of zoological type nomenclature: a reference source. Bishop Museum Technical Report 41: 1-25.

Frost, C.D. \& A.W. Snoke (1989). Tobago, West Indies, a fragment of a Mesozoic oceanic island arc: petrochemical evidence. Journal of the Geological Society, London 146: 953-964; http://dx.doi. org/10.1144/gsjgs.146.6.0953

Hanken, J. (1982). Appendicular skeletal morphology in minute salamanders, genus Thorius (Amphibia: Plethodontidae): growth regulation, adult size determination, and natural variation. Journal of Morphology 174: 57-77.

Hanken, J. \& D.B. Wake (1993). Miniaturization of body size: organismal consequences and evolutionary significance. Annual Review of Ecology, Evolution, and Systematics 24: 501-519.

Hanken, J. \& D.B. Wake (1994). Five new species of minute salamanders, genus Thorius (Caudata: Plethodontidae), from northern Oaxaca, Mexico. Copeia 1994(3): 573-590.

Hardy, J.D., Jr. (2001). A new frog of the genus Eleutherodactylus from the Island of Tobago, West Indies. Bulletin of the Maryland Herpetological Society 37: 25-31.

Hedges, S.B., W.E. Duellman \& M.P. Heinicke (2008). New World direct-developing frogs (Anura: Terrarana): molecular phylogeny, classification, biogeography, and conservation. Zootaxa 1737: $1-182$

Heyer, W.R., M.A. Donnelly, R.W. McDiarmid, L.C. Hayek \& M.S. Foster (Eds.) (1994). Measuring and Monitoring Biological Diversity: Standard Methods for Amphibians. Smithsonian Institution Press, Washington, USA, 364pp.

Hoyos, J.M., C. Mantilla, D. Galindo \& L. Salgar (2014). Phylogenetic analysis whithin [sic] the Pristimantis unistrigatus (Anura, Craugastoridae) group based on morphological characters. Caldasia 36(1): 107-124.

IUCN (2001). IUCN Red List Categories and Criteria: Version 3.1. IUCN Species Survival Commission. IUCN, Gland, Switzerland and Cambridge, United Kingdom.

Jowers, M.J., R.M. Lehtinen, R.J. Downie, A.P. Georgiadis \& J.C. Murphy (2014). Molecular phylogenetics of the glass frog Hyalinobatrachium orientale (Anura: Centrolenidae): evidence for Pliocene connections between mainland Venezuela and the island of Tobago. Mitochondrial DNA, Early Online 1-6; http://dx.doi.org/ 10.3109/19401736.2014.880888

Kaiser, H. (1993). Systematics and Biogeography of Eastern Caribbean Frogs. Unpubl. PhD Thesis, McGill University, Montréal, Québec, Canada.

Kaiser, H. (1994). Two origins for Eastern Caribbean Eleutherodactylus (Anura: Leptodactylidae): molecular evidence and its biogeographic consequences. Abstract, 74th Annual Meeting of the American 
Society of Ichthyologists and Herpetologists, Los Angeles, California, USA

Kaiser, H. (1996). Systematics and biogeography of Eastern Caribbean Eleutherodactylus (Anura: Leptodactylidae): consensus from a multidisciplinary approach, pp. 129-140. In: Powell, R. \& R.W. Henderson (eds.). Contributions to West Indian Herpetology: a Tribute to Albert Schwartz. Society for the Study of Amphibians and Reptiles, Ithaca, New York, USA.

Kaiser, H. (1997). Origins and introductions of the Caribbean frog, Eleutherodactylus johnstonei (Leptodactylidae): management and conservation concerns. Biodiversity and Conservation 6: 1391-1407.

Kaiser, H., D.M. Green \& M. Schmid (1994a). Systematics and biogeography of Eastern Caribbean frogs (Leptodactylidae: Eleutherodactylus), with the description of a new species from Dominica. Canadian Journal of Zoology 72(2): 2217-2237; http:// dx.doi.org/10.1139/z94-297

Kaiser, H., J.D. Hardy, Jr. \& D.M. Green (1994b). Taxonomic status of Caribbean and South American frogs currently ascribed to Eleutherodactylus urichi (Anura: Leptodactylidae). Copeia 1994(3): 780-796.

Kaiser, H., C.M. Dwyer, W. Feichtinger \& M. Schmid (1995). A new species of Eleutherodactylus (Anura: Leptodactylidae) from Tobago, West Indies and its morphometric and cytogenetic characterization. Herpetological Natural History 3: 151-163.

Lehr, E. \& L.A. Coloma (2008). A minute new Ecuadorian Andean frog (Anura: Strabomantidae, Pristimantis). Herpetologica 64(3): 354367; http://dx.doi.org/10.1655/07-089.1

Lynch, J.D. \& W.E. Duellman (1997). Frogs of the genus Eleutherodactylus (Leptodactylidae) in Western Ecuador: systematics, ecology, and biogoegraphy. Special Publication of the Museum of Natural History, University of Kansas 23: 1-236.

Lynch, J.D. \& E. La Marca (1993). Synonymy and variation in Eleutherodactylus bicumulus (Peters) from northern Venezuela, with a description of a new species (Amphibia: Leptodactylidae) Caribbean Journal of Science 29: 133-146.

Maggenti, M.A.B., A.R. Maggenti \& S.L. Gardner (Eds.) (2005). Online Dictionary of Invertebrate Zoology. http://digitalcommons.unl.edu/ onlinedictinvertzoology/2/. Accessed on 17 March 2015.

Manzanilla, J., M.J. Jowers, E. La Marca \& M. García-París (2007). Taxonomic reassessment of Mannophryne trinitatis (Anura: Dendrobatidae) with a description of a new species from Venezuela. Herpetological Journal 17(1): 13-42.

MacArthur, R.H. \& E.O. Wilson (1967). The Theory of Island Biogeography. Princeton University Press, Princeton, New Jersey, USA, 203pp.

Mijares-Urrutia, A., J.C. Señaris \& A. Arends (2000). Taxonomía de algunos microtéidos (Squamata) de Venezuela, I: Variación y distribución geográfica de Euspondylus del nordeste de Venezuela. Revista de Biología Tropical 48: 671-680.

Pérez, M.H.S. (2014). Standard Symbolic Codes for Institutional Resource Collections in Herpetology and Ichthyology: an Online Reference. Version 5.0 (22 September 2014). American Society of Ichthyologists and Herpetologists, Washington, D.C., USA.
Rivas F.G., E. La Marca \& O. Oliveros (1999). Una nueva especie de Anadia (Reptilia: Sauria: Gymnophthalmidae) del noreste de Venezuela. Acta Biologica Venezuelica 19(4): 27-32.

Rivas, G., W.E. Schargel \& J.M. Meik (2005). A new species of Riama (Squamata: Gymnophthalmidae), endemic to the Península de Paria, Venezuela. Herpetologica 61(4): 461-468; http://dx.doi. org/10.1655/04-96.1

Rivas, G.A., P.M. Sales Nunes, J.R. Dixon, W.E. Schargel, J.R. Caicedo, T.R. Barros, E.G. Camargo \& C.L. Barrio-Amorós (2012). Taxonomy, hemipenial morphology, and natural history of two poorly known species of Anadia (Gymnophthalmidae) from northern South America. Journal of Herpetology 46(1): 33-40; http://dx.doi. org/10.1670/10-139

Rivero, J.A. (1961). Salientia of Venezuela. Bulletin of the Museum of Comparative Zoology 126: 1-207.

Rivero, J.A. (1964). The distribution of Venezuelan frogs. Caribbean Journal of Science 4: 307-318.

Schargel, W.E., G. Rivas Fuenmayor \& C.W. Myers (2005). An enigmatic new snake from cloud forest of the Península de Paria, Venezuela (Colubridae: Genus Taeniophallus ?). American Museum Novitates 3484: 1-24; http://dx.doi.org/10.1206/00030082(2005)484[0001:AENSFC]2.0.CO;2

Schmid, M., C. Steinlein \& W. Feichtinger (1992). Chromosome banding in Amphibia. XVII. First demonstration of multiple sex chromosomes in amphibians: Eleutherodactylus maussi (Anura, Leptodactylidae). Chromosoma 101(5-6): 284-292; http://dx.doi. org/10.1007/BF00346007

Schmid, M., W. Feichtinger, C. Steinlein, T. Haaf, M. Schartl, R. Visbal García, J. Manzanilla Pupo \& A. Fernández Badillo (2002). Chromosome banding in Amphibia. XXVI. Coexistence of homomorphic $X Y$ sex chromosomes and a derived $Y$-autosome translocation in Eleutherodactylus maussi (Anura, Leptodactylidae). Cytogenetics and Genome Research 99(1-4): 330-343; http:// dx.doi.org/10.1159/000071612

Schmid, M., W. Feichtinger, C. Steinlein, R. Visbal García \& A. Fernández Badillo (2003). Chromosome banding in Amphibia. XXVIII. Homomorphic $X Y$ sex chromosomes and a derived $Y$-autosome translocation in Eleutherodactylus riveroi (Anura, Leptodactylidae). Cytogenetics and Genome Research 101(1): 62-73; http://dx.doi. org/10.1159/000073420

Schmid, M., C. Steinlein, J.P. Bogart, W. Feichtinger, P. León, E. La Marca, L.M. Diaz, A. Sanz, S.-H. Chen \& S.B. Hedges (2010). The chromosomes of terraranan frogs: insights into vertebrate cytogenetics. Cytogenetics and Genome Research 130-131: 1-568.

Terán-Valdez, A. \& J.M. Guayasamin (2010). The smallest terrestrial vertebrate of Ecuador: a new frog of the genus Pristimantis (Amphibia: Strabomantidae) from the Cordillera del Cóndor. Zootaxa 2447: 53-68.

Walker, C.F. \& F.H. Test (1955). New Venezuelan frogs of the genus Eleutherodactylus. Occasional Papers of the Museum of Zoology, University of Michigan 561: 1-12.

Yeh, J. (2002). The effect of miniaturized body size on skeletal morphology in frogs. Evolution 56(3): 628-641; http://dx.doi. org/10.1111/j.0014-3820.2002.tb01372.x 
Resumen (Spanish Abstract): Trabajo de campo en el bosque nublado de la remota Península de Paria en el extremo noreste de Venezuela en 2001 llevó al hallazgo de varios ejemplares que podían sin ninguna duda referirse al género Pristimantis. Análisis subsiguientes de material comparativo de varios museos llevó el total de ejemplares colectados en esa área a 44, que representan cinco nuevas especies. Dos de ellas, Pristimantis geminus sp. nov. y $P$. nubisilva sp. nov., poseen un fenotipo parecido a la especie de Trinidad $P$. urichi, apoyando la predicción de que los Pristimantis del NE continental de Suramérica probablemente son las formas que originaron especies de la isla de Trinidad. Pristimantis hoogmoedi sp. nov. es fácilmente identificable por su gran tamaño y ojos rojos. Dos de las especies, $P$. longicorpus sp. nov. y P. pariagnomus sp. nov., son muy distintivas morfológicamente pero sólo conocidas por los holotipos. La primera se reconoce por la forma del cuerpo alargado y extremidades relativamente cortas, mientras que el último tiene una morfología manual muy distintiva y probablemente sea la especie de rana más pequeña de Venezuela. Estudios de bandeo de cromosomas de $P$. nubisilva sp. nov. y $P$. hoogmoedi sp. nov. revelaron un numero de cromosomas $2 n=36$ y $2 n=26$, respectivamente, con una inusual fusión submetacentrica de cromosomas 11;18 en algunos machos de la primera especie y en un pareo meiótico único de cromosomas en los machos de la ultima especie. Las cinco especies pueden ser diferenciadas fácilmente por su osteología, así como por la extensión del esfenetmoide y otras características en el techo de la boca, así como por la forma y arreglo de los elementos mesopodiales. La inesperada alta diversidad simpátrica de Pristimantis en la región, junto al alto grado de endemicidad de reptiles y anfibios, posicionan a la Península de Paria como un centro de diversidad de ranas en la región. La similitud de algunas especies de Paria con aquéllas de Trinidad, Tobago, y la Cordillera de la Costa Central representa una pieza de evidencia tangible sobre el vínculo biogeográfico de estas dos áreas.

Palabras clave: Pristimantis, Península de Paria, Venezuela, nuevas especies, Trinidad, Tobago, taxonomía, biogeografía, osteología, cromosomas.

Acknowledgments: Our thanks go to Marinus Hoogmoed and Stefan Gorzula for allowing us to use the specimens they collected 35 years ago for our analysis. Jason Trujillo and Walter Schargel were delightful and competent companions in the field. We applaud the artistic talent of hikE Worth (spelled artistically correctly in this manner), who was able to create very nice drawings of hands and feet with instructions emailed from across the Atlantic. We thank Mark O'Shea for editing the frog photos and for his help with producing the maps, and Mayke De Freitas and Walter Schargel for their suggestions to improve our conservation section and resumen, respectively. For coordinating the use of $\mathrm{x}$-ray equipment at the USNM and instruction in how to obtain high-quality digital images we thank Jeremy Jacobs and Sandra Raredon. For sending, receiving, and tracking specimen loans, we are very grateful to Tito Barros (MBLUZ), Hedelvy Guada and Mercedez Salazar (MBUCV), Celsa Señaris (MHNLS), and Robert Wilson (USNM). Esther Dondorp (RMNH) provided us with copies of field notes for the collection made in 1978, and checked RMNH specimen numbers. GRF thanks the Fundación Proyecto Paria for logistical support, and particularly for the use of a vehicle, during several of his trips to the region.

Author Contributions: HK and CLB designed the project, conducted fieldwork, and co-wrote the manuscript. HK contributed additional comparative characteristics from work on museum specimens, added morphometric data and their analysis, and conducted the osteological analysis. GR did fieldwork, added natural history comments, and wrote most of the conservation section. CS completed the chromosome preparation and staining. MS prepared the chromosome figures and contributed the chromosome section. CLB and GR reviewed and edited the completed manuscript.

Author Details: DR. HINRICH KAISER is a Professor in the Department of Biology at Victor Valley College in Victorville, California, USA and a Research Collaborator of the United States National Museum of Natural History, Smithsonian Institution, Washington, D.C., USA. He currently serves as an Associate Editor with both Journa of Herpetology and Herpetology Notes, and as a member of the International Advisory Board of the Foundation for Post-Conflict Development, New York. Hinrich's most recent publications have focused on the herpetofauna of Timor-Leste and nearby areas of Wallacea, as well as on the defense of herpetological taxonomy against taxonomic vandalism. CÉSAR L. BARRIO AMORós is a freelance researcher and photographer and the owner of Doc Frog Expeditions and Photography. His research focus is on the taxonomy of Venezuelan amphibians and reptiles, especially Terrarana and Dendrobatoidea. He has published or coauthored more than 200 articles, including the description of 50 mainly Venezuelan new species. César currently resides in Costa Rica, where he teaches herpetology courses and leads nature tours with a herpetological emphasis. GILSON A. RIVAS earned a BSc in Animal Science from Fundación La Salle de Ciencias Naturales and currently serves as co-editor of the journal Anartia in the Museo de Biología, Facultad Experimental de Ciencias, Universidad del Zulia, Maracaibo, Venezuela. He has been involved in research on the systematics and conservation of the Venezuelan herpetofauna for two decades, having co-authored more than 100 scientific contributions, including descriptions of over 20 new species of amphibians and reptiles. Gilson's research focus is the herpetofauna of the Península de Paria and surrounding areas, particularly with respect to systematics and conservation, as well as the influence of invasive species and human development and their impact on the native fauna. CLAUS STEINLEIN holds an engineering degree from the University of Applied Sciences, Hamburg, Germany. His unrivaled expertise in the development of cytogenetics techniques and protocols has enabled him to produce exceptional chromosome data sets over the past three decades, leading to his co-authorship of numerous research articles in the highest ranked scientific journals. He currently serves as Editorial Administrator of the journals Cytogenetic and Genome Research, Sexual Development, and Molecular Syndromology. DR. MICHAEL SCHMID obtained his doctorate in human biology from the University of Ulm, Germany. He directs the cytogenetics group in the Department of Human Genetics, University of Würzburg, Germany, where he has been a professor since 1995. Aside from his main line of research in human genetics, Michael has broad interest in vertebrate cytogenetics, and his series of papers on chromosome banding in Amphibia remains a standard setting contribution. He is Editor-in-Chief of the journals Cytogenetic and Genome Research, Sexual Development, and Molecular Syndromology. He was one of the founding editors of the journal Chromosome Research. He currently serves as Editor of the revived book series Monographs in Human Genetics and as Executive Editor of the new book series Genome Dynamics. 